\title{
Skeleton Based Perpendicularly Scanning - A New Scanning Strategy for Additive Manufacturing: Modeling and Optimization
}

Mostapha El Jai ( $\square$ m.eljai@ueuromed.org )

Université Euro-Méditerranéenne de Fès: Universite Euro-Mediterraneenne de Fes

latimad AKHRIF

Euromed Institute of Technology, Euromed University of Fez

Nourddin Saidou

INSA-Euromed, Euromed University of Fez

Original Article

Keywords: Skeleton Based Scanning, chess scanning strategy, Additive manufacturing, production time, gain of time

Posted Date: November 25th, 2020

DOI: https://doi.org/10.21203/rs.3.rs-113295/v1

License: (9) This work is licensed under a Creative Commons Attribution 4.0 International License. Read Full License 


\title{
Skeleton Based Perpendicularly scanning: A New scanning strategy for Additive Manufacturing: Modeling and Optimization
}

\author{
El Jai ${ }^{(1, *)}$, M., Akhrif, I. ${ }^{(1)}$, and Saidou, N. ${ }^{(2)}$ \\ ${ }^{(1)}$ Euromed institute of technology, Euromed University of Fez, Morocco \\ ${ }^{(2)}$ INSA-Euromed, Euromed University of Fez, Morocco
}

Address: Route Nationale Fez-Meknes, Fez

*Corresponding author. Email: m.eljai@ueuromed.org

\begin{abstract}
In this paper, the authors propose a novel strategy of 2D scanning that might be adapted for any additive manufacturing process. The featured novelty corresponds to a Skeleton Based Perpendicularly (SBP) of the 2D shape of each slice. Thus, it is proposed to minimize the total production time of a given layer under some constraints. In other word, it is proposed to study the competitiveness conditions of the new scanning technique regarding the classical chess scanning strategy from a productivity perspective. In order to introduce this new technique, the paper treats the case of a rectangular layer. The competitiveness of the proposed technique was discussed according to chess decomposition parameters, the hatch space distance, and the dimensions of the primitive rectangle layer to analyze. The indicators introduced corresponds to "the gain of production time" and "the specific gain of production time per surface unit"; then, these latter were computed and discussed in two separated cases of study. The findings show that, by the adoption of the SBP technique instead the chess scanning strategy, it is possible to save about $3 \%$ to $45 \%$ of production time gain for the first case of study. The gain of production time per surface unit was analyzed in the second case of study. The correspondent analysis permitted to highlight the percentage of gain of time related to the area to scan. Indeed, the gain per surface unit varies between $4.32 \times$ $10^{-6} \% / \mathrm{mm}^{2}$ and $6.96 \times 10^{-05} \% / \mathrm{mm}^{2}$. In one hand, these indicators depend linearly on the decomposition strategy of the central area of the SBP technique and also according to the rectangle dimensions. In addition, for the lowest values of hatch space, around $25 \mu \mathrm{m}$, the two techniques in competition present quasi-similar production time, where the variations between them is minimal. Nevertheless, starting from $65 \mu \mathrm{m}$, the SBP scanning strategy present considerable less time of production judged as exponentially decreasing according to the hatch space distance. Finally, one can see that the scanning model proposed could present major contributions in other scientific and technical fields that use surface control as territorial security, water adduction and distribution, telecommunication, etc. by varying and adapting the decision variables according to each field of study.
\end{abstract}

\section{Notations}

Skeleton Based Scanning, chess scanning strategy, Additive manufacturing, production time, gain of time.

\section{Introduction and context}

Additive manufacturing is now considered as one of the major manufacturing processing classes that allows to build complex volume and surface geometries. The Additive manufacturing market size knew a phenomenal growth in the last decade. According to Wohlers Associates (2016), the turnover evolved exponentially between 2009 and 2015; in 2009 the market size was evaluated at 1.1 Billion of US Dollars and the growth reached around $500 \%$ reaching 5.1 Billion of US Dollars in 2015 [1]. Additive manufacturing is applied to most industries and the corresponding processes are dedicated especially to complex designed parts [2-5]. These latter are used for structural features or as decorative utensils alike. Currently, the construction industries are also more and more interested by $3 \mathrm{D}$ printing since it will permit to include oriented mechanical properties and complex geometries [6-8].

Coupled to topology optimization (TO) and generative design (GE), AM processes are nowadays used to materialize very complex parts designed by TO and GE techniques. From a performance stand-point, an optimized choice of the process parameters combinations leads to obtain the target performance and to reach a given level of 
final mechanical and material features. Indeed, researchers tried to explain the dependencies and interdependencies between the process parameters: process outputs according to process inputs [5, 9-16].

In general, semi-empirical models were used in order to model and to characterize the AM processes taking advantages from existing physical phenomena and specific engineering areas (welding, sintering, diffusion, metallurgy, polymers, etc.). In addition, the designations of the AM processes are proposed by the manufacturers according to physical and technical specifications [17].

Since the AM processes use printing layer per layer, the layer's characteristics could be considered as common features for the majority of AM technologies. In general, these characteristics are:

- The layer thickness,

- Physics of scanning,

- Geometry parameters of the scanning strategy

These latter are chosen according to each technology and machine precision. For instance, the layer thickness in Selective Laser Melting (SLM) varies from $20 \mu \mathrm{m}$ to $50 \mu \mathrm{m}[18,19]$, while in Selective Laser Sintering (SLS), the thickness varies from $16 \mu \mathrm{m}$ to $150 \mu \mathrm{m}$. Nevertheless, some researchers gone up to $280 \mu \mathrm{m}$ in order to qualify the effect of the powder layer thickness on the morphological and mechanical properties of the produced parts [20].

Except layer properties and scanning strategies, the input parameters varied as the physical phenomena to master. For instance, in SLS and SLM, the major inputs correspond in general to the laser properties and heat effects, while for the Fused Deposition Modeling (FDM), the inputs concern mainly the physics of extrusion.

The support and scanning strategies are also important parameters that have great influences on the heat transfer from the built material to the printing plate generating residual stresses or deflection of the printed layer or part. That is why the support structures influence the final microstructure and consequently the mechanical behavior of the final products $[21,22]$.

The post-process analysis concerns in general the correlations between the process inputs and:

- the mechanical properties:

- Static resistance $[23,24]$

- Tolerance of the products in term of roughing and finishing need [25-27]

- Fatigue behavior [28-30]

- Metallurgical properties and chemical resistance [31-34].

Practically, the effects of the input parameters are coupled, and it would be not so easy to control each parameter apart. Thus, for AM processes, controlling the interactions between the input parameters is a key to control the whole "geometry-material-process" interactions. That is why, researchers tried to understand the complexity of the inputs interactions using design of experiments and robust statistical method. Taguchi approach is widely used to reach this objective as a black box approach to summarize the manufacturing process control $[9,11,14-16]$.

Moreover, scanning strategies are a set of those inputs that influence directly the process outputs in terms of:

- $\quad$ density of the locally processed matter [12, 19]

- residual stress generated during the printing [12, 32, 34, 35]

- heat effect of the neighbor areas during the processing [12, 35-37]

- mechanical anisotropy according to the scanning direction [23, 24, 37]

- $\quad$ resulting microstructures $[33,34,37]$

Hence, scanning strategies should be considered as a vector of parameters that have the following components:

- pattern style [10]

- $\quad$ orientation and placement strategies $[13,38]$

- hatch distance and orientation [13,38] 
From a technical perspective, the hatch distance $(e)$ must be greater than or equal to the resolution of the printer $\left(e_{r e s}\right)$, but less than a critical value $\left(e_{c}\right)$ that characterizes some real process constraints.

For instance, in the SLM technology, two major effects that occur during and after the laser shot condition the printed part characteristics [39]:

- $\quad$ The melting/solidification cycle of the metal that generates the melted zone appearing like a weld seam. The width of the molten zone is assessed by different methods of measurement: IR camera measurement, measurement of the width of the remelted zone $\left(D_{M Z}\right)$ on the surface of the substrate with an optical microscope and the width of the Heat Affected Zone (HAZ) including remelted zone;

- The HAZ that could be expressed as a function the laser scanning speed " $V$ ", the thermal diffusivity " $a$ " and the laser beam diameter " $\mathrm{d}$ ".

The modeling of the heat affected zone elaborated by Andreyavna et al. (2015) leads to propose a preliminary value for the parameter $e_{c}$ discussed above [39]:

- $\quad e_{c}>H A Z$ : two parallel and adjacent laser spots would not have interdependent effects since the hatch distance $e_{c}$ distance is important;

- $\quad D_{M Z} \leq e_{c} \leq H A Z$ : for two parallel and adjacent laser spots, the HAZ and the melted zone that are realized the first will be heat-treated by the thermal effect of the second laser beam. This is called intrinsic heat treatment (IHT) [10];

- $\quad 0<e_{c} \leq D_{M Z}$ : the melted zone will be remelted partially by the second laser scanning.

In general, the choice of the parameter $e_{c}$ affects the homogeneity of the material, its density, and consequently the mechanical and physical properties. In the literature, the choice of the hatch distance is in general taken into account as a process parameter that belong to hatch style characteristics [38].

In this paper, the authors present the mathematical design and formulation of a new scanning strategy. The proposed technique is based on the skeleton construction of $2 \mathrm{D}$ shapes. It corresponds to a scanning perpendicularly to the skeleton of the borders of the shape that has to be generated in a previous step by a $p$ reprocess slicing algorithm. Then, this new strategy will be named "Skeleton Based Perpendicularly" (SBP) strategy. Sect. 2.1 presents a little overview on what a skeleton of a shape is.

Since the SBP scanning technique is proposed for the first time, the paper will treat the case of a rectangle as a simple geometry to analyze. Mainly, the study tries to enhance the productivity of a given AM process by minimization the total displacement along the solid filled region of a layer. The total productive time is then the summation of all production times.

This work compares the classical chess pattern, that is used in the existing AM technologies, to the proposed SBP scanning strategy by the mean of a gain of production time modeling. Further works will focus on more complex geometries and other will be dedicated to mechanical properties of printed parts by this new design. Consequently, in order to simulate the total scanning lengths and production times, it is proposed to apply the methodology developed in the next sections by adopting SLM process parameters in the cases of study. Table 1 groups the input parameters that have been used in the bibliography related to the SLM process.

Table 1 SLM filling parameters: Hatch space and scanning speed

\begin{tabular}{|c|c|c|c|c|}
\hline Hatch space $(\mathbf{m m})$ & Scanning speed $\mathbf{( m m} / \mathbf{s})$ & Laser power $\mathbf{( W )}$ & Material & Reference \\
\hline 0.150 & 800 & 195 & Al-Si-10Mg & {$[40]$} \\
\hline 0.090 & 2500 & 300 & Invar & {$[41]$} \\
\hline 0.300 & $333-1000$ & $180-200$ & Invar & {$[42]$} \\
\hline $0.08-0.320$ & $700-2200$ & $150-300$ & Invar & {$[43]$} \\
\hline 0.150 & $500-1800$ & $150-400$ & SS 316L & {$[44]$} \\
\hline $0.070-0.140$ & $300-1250$ & $50-100$ & SS 316L & {$[45]$} \\
\hline $0.050-0.070$ & 700 & 100,150 & SS 316L & {$[24]$} \\
\hline
\end{tabular}




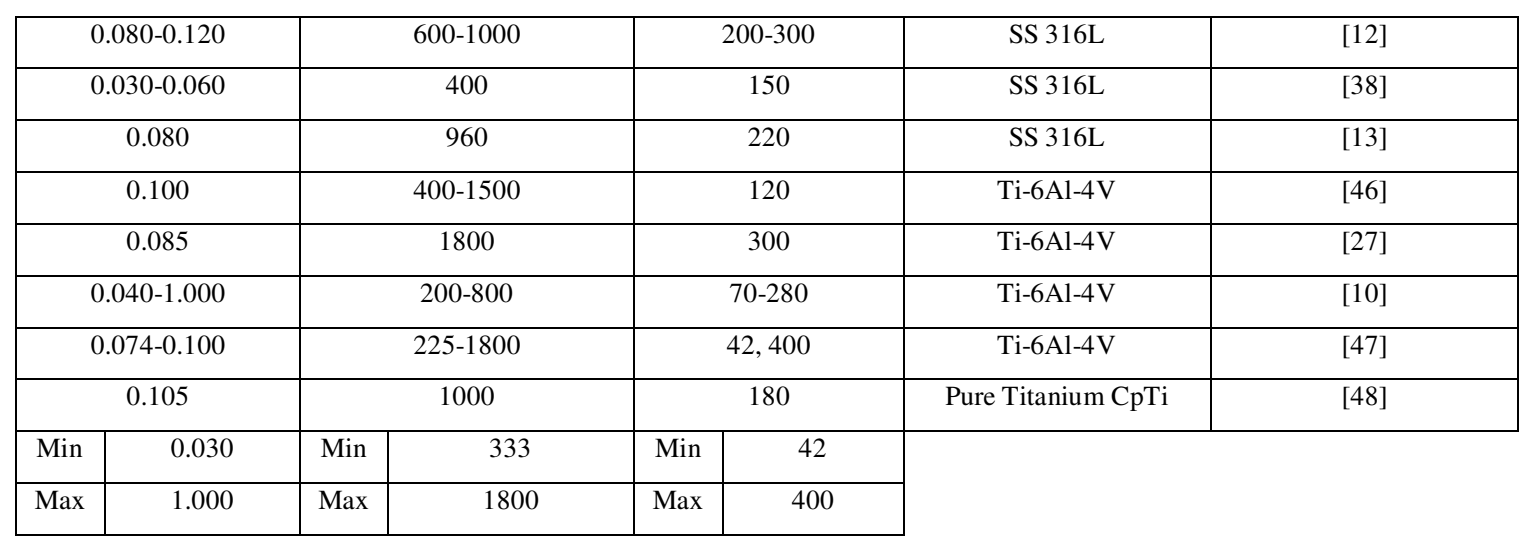

The rest of the paper will be organized as follows: the next section will discuss the mathematical modeling of the total length and total production time of both classical chess pattern and the new SBP pattern; the third section will present the optimization problem formulation related to the comparison of the strategies in competition; and the last section will conclude the work and present the future developments.

\section{Methodology}

The objective of the paper is to compare the productivity of the classical chess scanning pattern and the new scanning technique SBP that is based on the skeleton on the 2D-shapes that are generated in the slicing process.

For the two scanning strategies in competition, the SBP and chess strategies, the first step corresponds to choose the appropriate geometric parameters in order to model efficiently the productive and non-productive lengths and by the way the total times of production. From a process stand-point, the components of the total scanning or production time are:

- the productive time: that expresses the time during which the scanning or the production is activated (laser spot for example)

- the non-productive time: that corresponds to the inactive jumps of the laser (laser off) or the rapid displacement of a filament nozzle for FDM processes.

In order to make in practice the new scanning strategy, the coming analysis will discuss especially the case of a given AM process that uses laser as thermal energy. Hence, as discussed in the introduction section, the SLM process parameters were chosen. An energetic discussion is also presented in sect 2.5.2.

Hence, fig. 1 presents the flow map of the methodology in order to simplify the apprehend the next sections.

Before, starting the mathematical developments, it is necessary to give a brief sight on what a mathematical skeleton of a shape is.

\subsection{Geometrical Skeleton definition}

The skeleton of a complex shape construction represents a fieldwork of different researchers. It is based on several studies and applications, such as the reconstruction of forms and image processing. This geometrical feature of a given $2 \mathrm{D}$ or $3 \mathrm{D}$ area finds its origin as a topological object of these latter $[49,50]$. The skeleton represents a physical or virtual object for the reconstitution of any form. It is premised upon a body of research in biology and physics. The skeleton has many applications, especially in the field of recognition of shapes and imagery [51, 52]. In 2-dimensions analysis, the skeleton is built or computed as the set of maximal discs of a given shape, and in 3dimensions as the set of maximal ball of the volume to skeletonize as displayed in fig. 2 [53]. It is then considered as a kind of a continuous line of barycenters. Consequently, the skeleton is, by its topological definition, the set of the nearest points from the frontier of a given shape [50]. It is worth mentioning that the first definition of the skeleton was proposed by Blum (1967) [54]; the skeleton was defined as the lines along which fire fronts meet when it started from the border of a given volume or layer [55]; the fire is propagated along the normal vector to 
the shape border. Fig. 3 shows the skeleton of a simple rectangle. Hence, the skeletonization is defined as an application that lies a given shape to its skeleton.

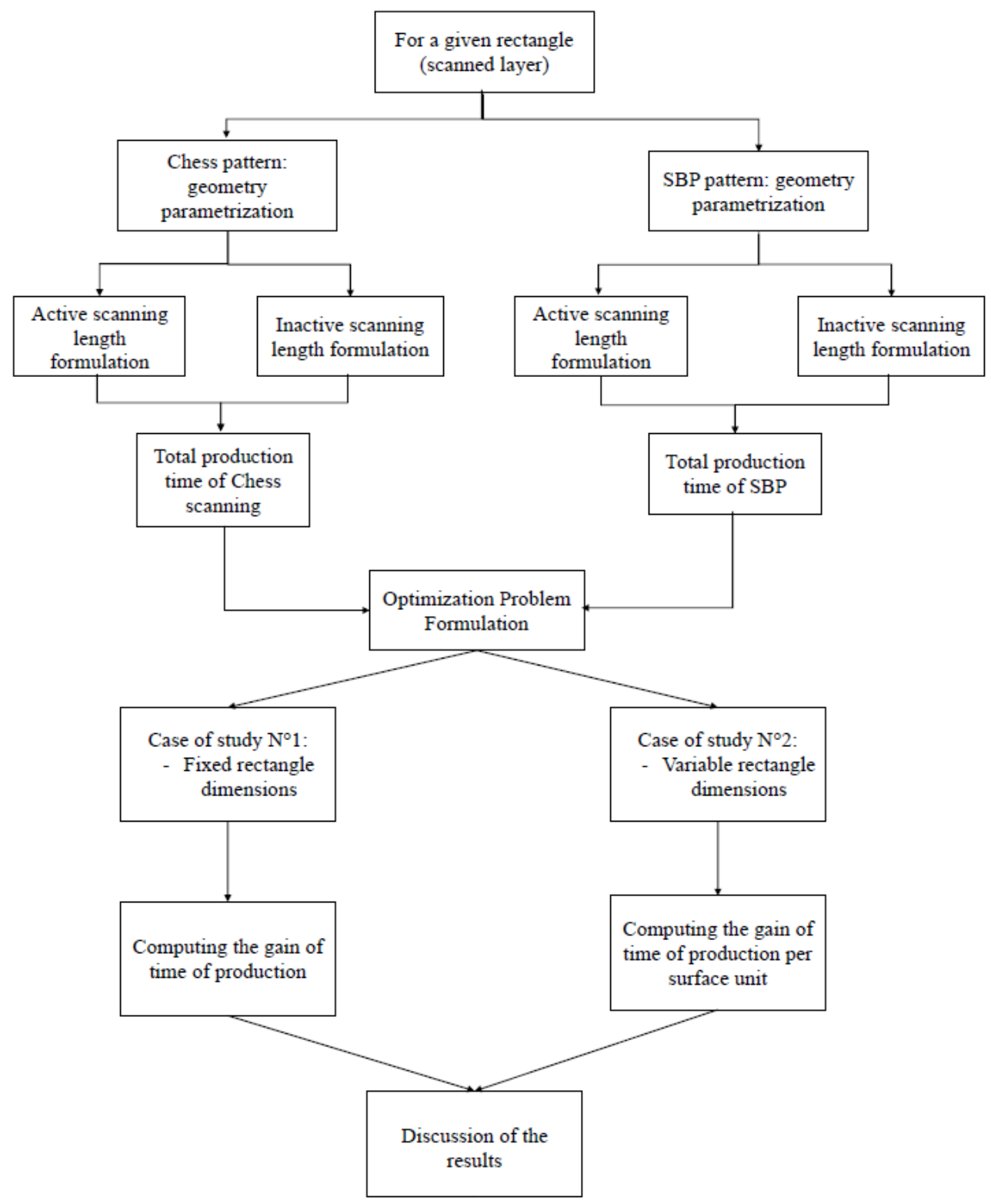

Fig. 1 Workflow of the study

For more details about the skeleton construction and use, the reader is referred to [50, 51, 55].

Given the above, this work was initiated by the idea that a scanning of a sliced layer in AM processes, starting from and to the skeleton, should allow the reduction scanning trajectory, and thus increase the productivity of the printing process.

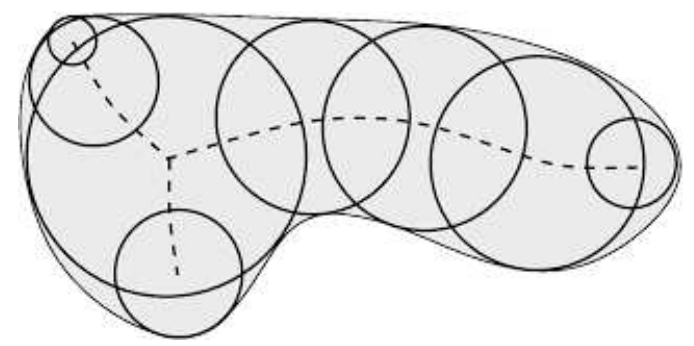

Fig. 2 Internal disks and maximal disks (balls) of a 2-D shape, Building the shape skeleton [53] 


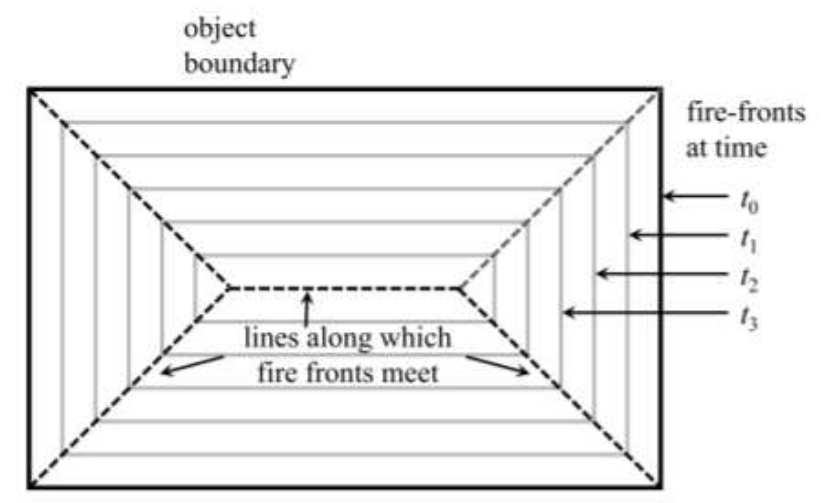

Fig. 3 Skeleton of a rectangle depicted by a set of lines as the lieu in which the fire fronts meet [55]

\subsection{Classical chess strategy: production time formulation 2.2.1. Geometry parametrization}

Fig. 4 presents a typical example of the classical chess scanning strategy.

According to this figure, the system (1) presents the possible count of pattern 1 (fig. 3) and pattern 2 (fig. 6). The system (1) expresses also the divisions of the $\left(L_{1} \times L_{2}\right)$ rectangle into regular $\left(n_{x} \times n_{y}\right)$ portions.

$$
\text { (1) }\left\{\begin{array}{l}
n_{x}=E\left(\frac{L_{1}}{a_{x}}\right) ; n_{x}=\frac{L_{1}}{a_{x}} \in \mathbb{N}^{*} ; a_{x}=\frac{L_{1}}{n_{x}} \\
n_{y}=E\left(\frac{L_{1}}{a_{x}}\right) ; n_{y}=\frac{L_{2}}{a_{x}} \in \mathbb{N}^{*} ; a_{y}=\frac{L_{2}}{n_{y}}
\end{array}\right.
$$

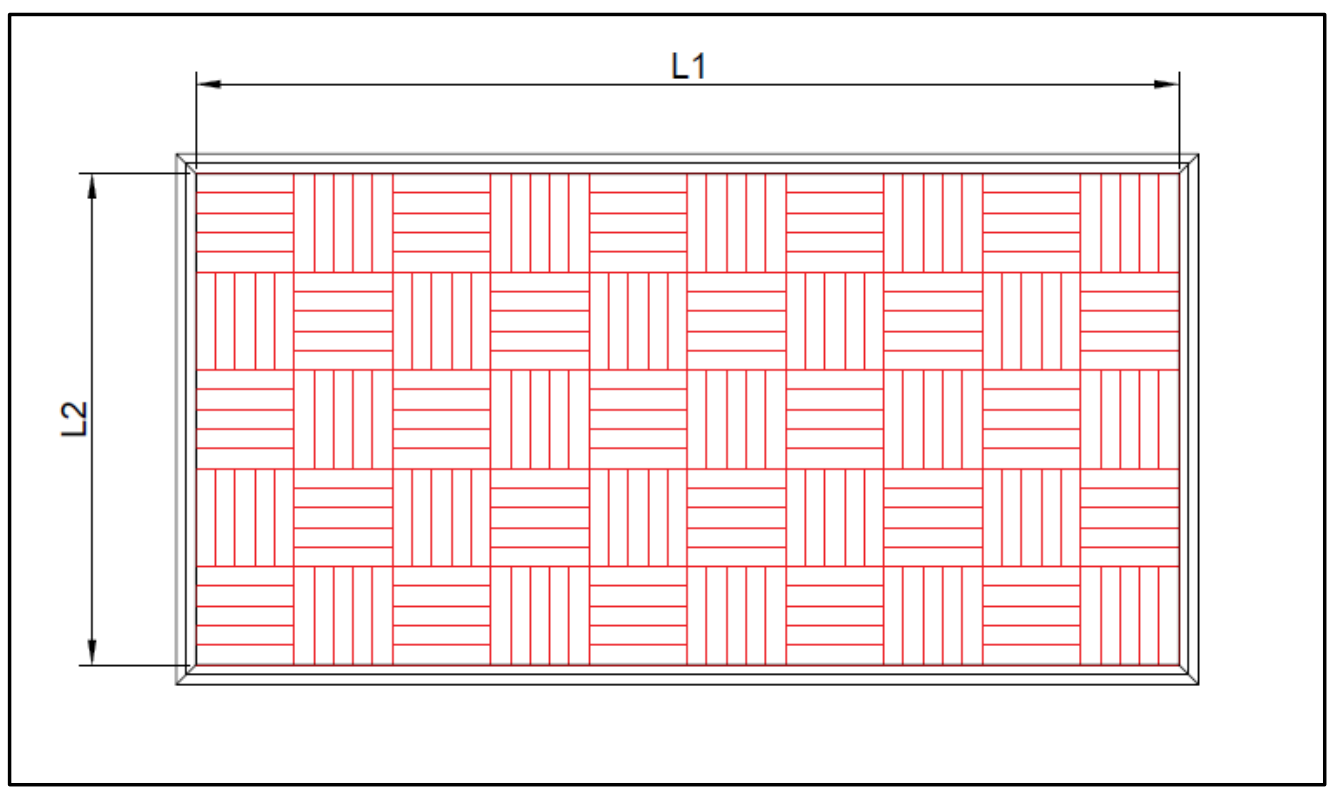

Fig 4 Classical Chess scanning policy in the Additive Manufacturing processes

Fig. 5 and 6 depicted the geometrical parametrization of the patterns of the chess scanning strategy as:

- $\quad a_{x}$ : length according to the $\vec{x}$ axis;

- $\quad a_{y}$ : length according to the $\vec{y}$ axis;

- $e$ : hatch space of the chess strategy. 


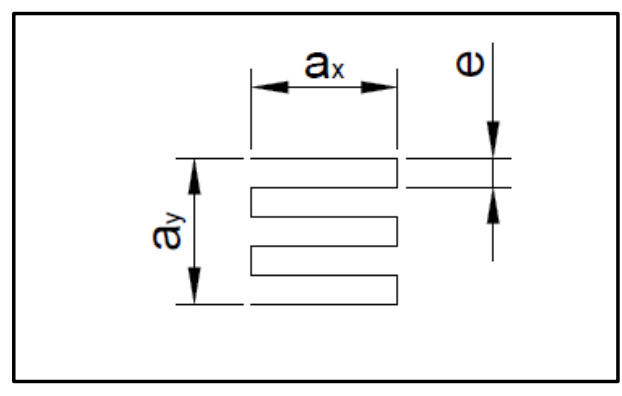

Fig 5 Chess scanning pattern $n^{\circ} 1$

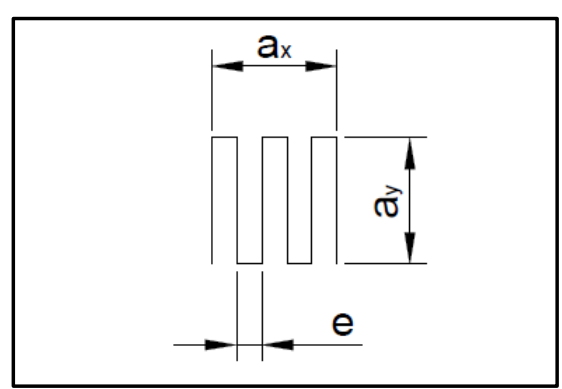

Fig 6 Chess scanning pattern $n^{\circ} 2$

The printing resolution is defined essentially as the minimal possible jump distance e permitted by the machine. That is to say that the hatch distance must be greater that the scanning resolution as expressed by the equation (2).

$$
e \geq e_{\text {res }}
$$

\subsubsection{Total scanning time}

It is worth mentioning that the developments details above concern especially the filling (solid) area of a given layer. That is to say that the borders building is not taken into account since it is considered to be the same for the two filling techniques that compete in this paper.

\section{Productive time: active scanning}

The productive time of the pattern 1 and pattern 2 could be determined directly from fig. 5 and 6 respectively.

The productive lengths of patterns 1 and 2 , are respectively noted as $L_{P_{1}}$ and $L_{P_{2}}$, and are expressed by the equations (3.1) and (3.2).

$$
\text { (3) }\left\{\begin{array}{l}
L_{P_{1}}=a_{x}\left(m_{y}+1\right) \\
L_{P_{2}}=a_{y}\left(m_{x}+1\right) \\
m_{x}=E\left(\frac{a_{x}}{e}\right) \\
m_{y}=E\left(\frac{a_{y}}{e}\right)
\end{array}\right.
$$

s.t.

$m_{x}$ and $m_{y}$ are respectively the number of divisions of the patterns 1 and 2 according to the axis $\vec{x}$ and $\vec{y}$.

\section{Non-Productive time: inactive scanning}

The inactive scanning could be defined as the scanning in which the laser is switched off for SLM or SLS processes. For FDM, this step is considered as productive. But since the cases of study adopted use SLM parameters, the designation of Non-Productive time and inactive scanning were adopted for the rest of the paper and it correspond to inactive jumps.

The non-productive lengths, $L_{N P_{1}}$ and $L_{N P_{2}}$, respectively related to the pattern 1 and pattern 2 are expressed by the expressions (4.1) and (4.2).

$$
\text { (4) } \begin{cases}L_{N P_{1}}=a_{y} & (4.1) \\ L_{N P_{2}}=a_{x} & (4.2)\end{cases}
$$

Thus, considering the productive scanning speed $V_{P}$ and non-productive speed (jump $e$ ) $V_{N P}$, the total production (scanning) time of each pattern $i$ is expressed by the equation (5).

$$
T_{i}=\left(\frac{L_{P}}{V_{P}}\right)_{i}+\left(\frac{L_{N P}}{V_{N P}}\right)_{i}
$$


Replacing the expressions (3) and (4) in (5) for pattern (1) and (2):

$$
\text { (6) }\left\{\begin{array}{l}
T_{1}=\frac{a_{x}}{V_{P}}\left(E\left(\frac{a_{y}}{e}\right)+1\right)+\frac{a_{y}}{V_{N P}} \\
T_{2}=\frac{a_{y}}{V_{P}}\left(E\left(\frac{a_{x}}{e}\right)+1\right)+\frac{a_{x}}{V_{N P}}
\end{array}\right.
$$

Thus the total scanning time of the filling area related to the chess scanning strategy in the case of a rectangle $L_{1} \times$ $L_{2}$ is given by the equation (7) and the system (8).

$$
\begin{aligned}
& T_{c h}=N_{1} T_{1}+N_{2} T_{2} \\
& \text { (8) }\left\{\begin{array}{c}
\text { if }\left(\left(n_{x} \text { is pair }\right) \text { or }\left(n_{y} \text { is pair }\right)\right): N_{1}=N_{2}=\frac{n_{x} n_{y}}{2} \\
\text { else }\left\{\begin{array}{c}
\text { if }\left(\begin{array}{c}
\text { the scanning begins } \\
\text { with pattern } 1
\end{array}\right): N_{1}=E\left(\frac{n_{x} n_{y}}{2}\right)+1 \\
\text { if }\left(\begin{array}{c}
\text { the scanning begins } \\
\text { with pattern } 2
\end{array}\right): N_{2}=E\left(\frac{n_{x} n_{y}}{2}\right)+1 \\
N_{1}+N_{2}=1
\end{array}\right.
\end{array}\right.
\end{aligned}
$$

Where

$N_{l}$ : is the total number of pattern 1 in the rectangle;

$N_{2}$ : is the total number of pattern 2 in the rectangle;

$n_{x}$ : is the number of divisions of the dimension of the rectangle along the axis $\vec{x}$;

$n_{y}$ : is the number of divisions of the dimension of the rectangle along the axis $\vec{y}$;

Remark Is considered a chess scanning beginning with a given pattern where this latter is positioned at the upperleft corner of a given rectangle zone. For instance, in figure 2, scanning begins with pattern 1.

So, the total productive and non-productive lengths related to the chess strategy can be derived from the expressions (3) and (4). One can prove easily the expression of productive and non-productive lengths as presented by the system (9).

$$
\text { (9) }\left\{\begin{array}{c}
L_{P_{c h}}=N_{1}\left(a_{x}\left(E\left(\frac{a_{x}}{e}\right)+1\right)\right)+N_{2} a_{2}\left(E\left(\frac{a_{2}}{e}\right)+1\right) \\
L_{N P_{c h}}=N_{1} a_{y}+N_{2} a_{x}
\end{array}\right.
$$

\subsection{Skeleton Based Perpendicularly strategy analysis}

In the SBP scanning strategy proposed, the skeleton is built for each sliced layer according to the contour of the layer. Thereafter, the SBP strategy operates by scanning perpendicularly to the skeleton. Fig. 7 presents a typical SBP scanning pattern for a $L_{1} \times L_{2}$ rectangle. The skeleton of the rectangle is depicted by the set of blue discrete lines. The areas A and B are more detailed in fig. 8 and 10.

Since the angles of the rectangle are equal to $90^{\circ}$, the bisector slices the angle to two equal angles of $45^{\circ}$. Indeed, according to fig. 5, an elementary geometrical identification permits to write the equations (10.1) and (10.2).

$$
\text { (10) }\left\{\begin{array}{l}
\alpha_{1} L_{1}=L_{2} / 2 \\
\alpha_{2} L_{1}=L_{1}-L_{2}
\end{array}\right.
$$




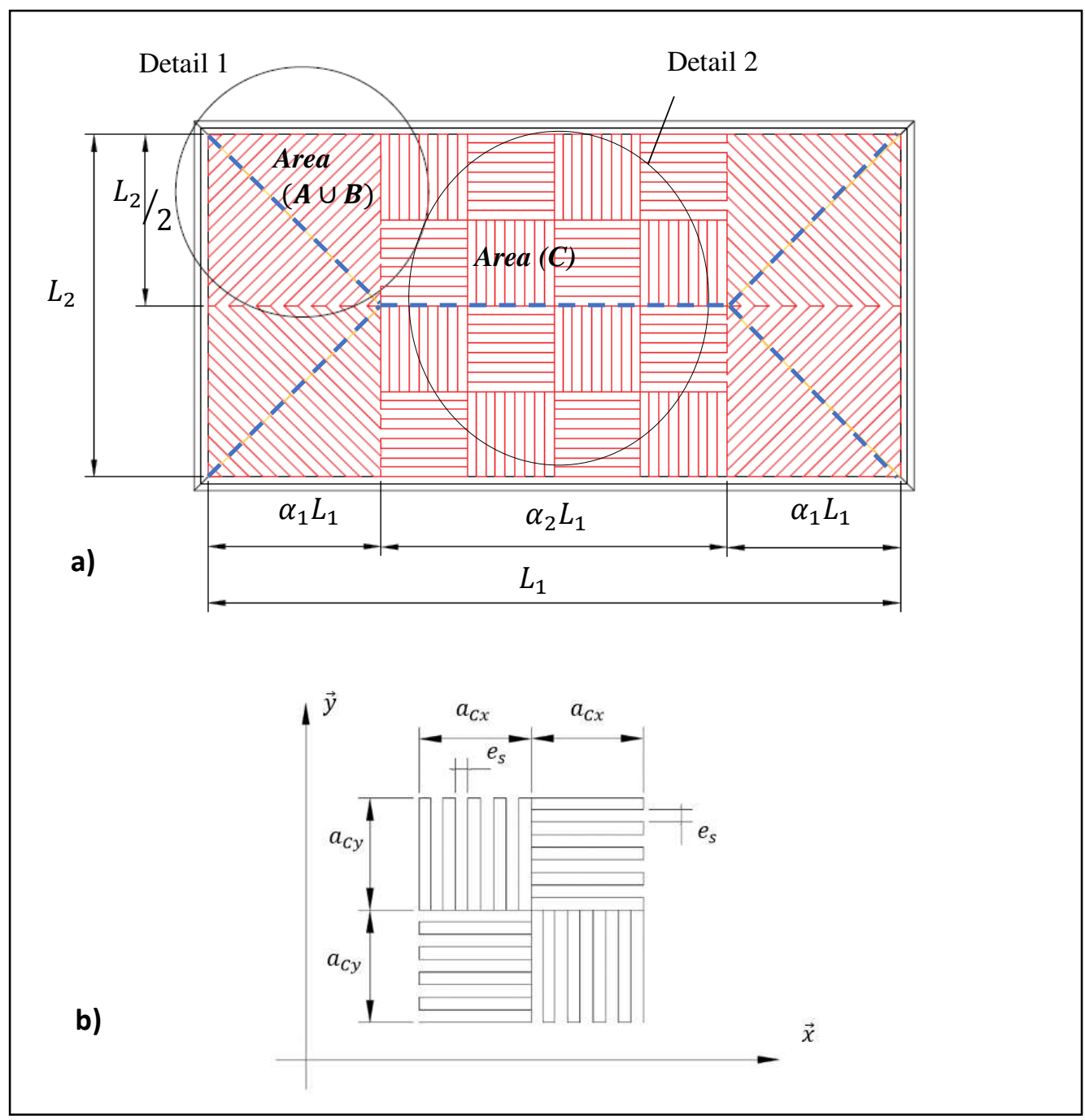

Fig 7 Skeleton Based Perpendicularly strategy scan pattern a) Global pattern b) C area pattern and the corresponding geometrical parameterization

The next paragraphs 2.3.1 to 2.3.3 present the formulation of the productive and non-productive lengths of the SBP strategy, according to the parameterization that is detailed in the fig. 7 to 9 .

In order to lighten the corpus of the article, the geometrical analysis and the algebraic developments were reported in the appendix.

\subsubsection{Geometrical parametrization and calculus variable}

Fig. 9 presents the characteristic points, vectors $(\vec{v}$ and $\vec{n})$ and bisector (B) necessary for the geometrical modeling of the scan distance series $\overline{C H} . \overline{C H}$ is considered as a characteristic distance of the problem because it describes the series of active scanning displacements. Once the distance $\overline{C H}$ expression is modeled, the computing of the total productive scanning $L_{P}$ of the areas $\mathrm{A}$ and $\mathrm{B}$ of the rectangle is direct; the total productive length corresponds to the summation of the series of $\overline{C H}_{i}$ as expressed by the equation (10).

$$
L_{P_{\text {Zone A or B }}}=\sum_{i \in\{\text { index set }\}} \overline{C H}_{i}
$$

So, the objective of the next paragraphs is to express $\overline{C H}_{i}$ as follows:

- $\quad$ Fig. 7 to 9 present the geometrical parameters that permit the calculation of the distances $\overline{C H}_{i}$; 


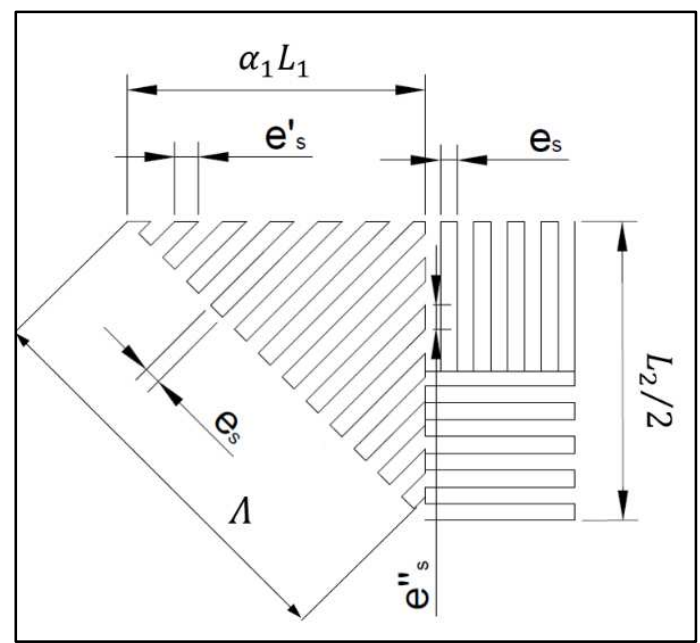

Fig 8 Detail 1 of fig. 7: Geometric parametrization of the (SBP) scanning strategy

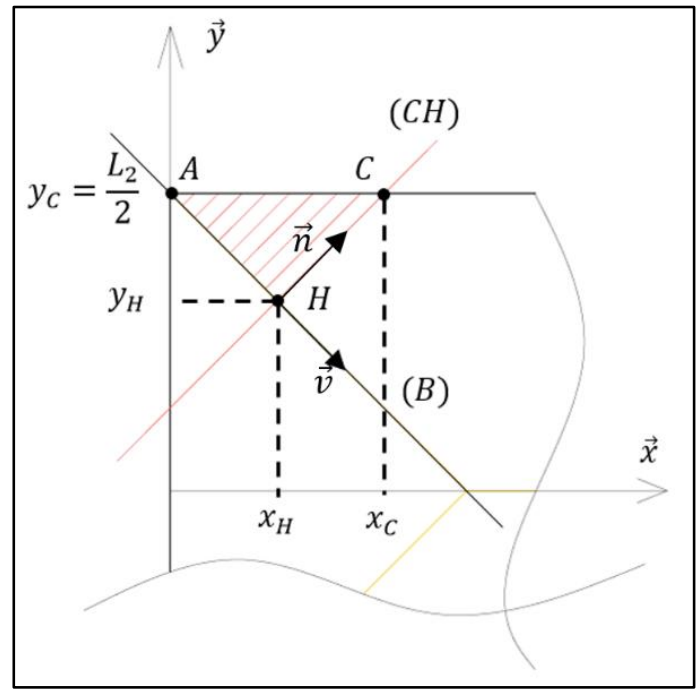

Fig 9 Positions and coordinates of the characteristic points $\{\mathrm{A}, \mathrm{C}, \mathrm{H}\}$

- The parameters and notations utilized are:

- $\left(x_{C}, y_{C}\right)$ and $\left(x_{H}, y_{H}\right)$ : resp. are the coordinates of the points $\mathrm{C}$ and $\mathrm{H}$;

- $\vec{n}$ : unitary vector that is normal to the skeleton at any point and it is also the unitary vector of the line $(\mathrm{CH})$;

- $\vec{v}$ : carrier vector of the bisector (B), it is perpendicular to the vector $\vec{n}$;

- The bisector at the up-left area of the rectangle is denoted (B). Its equation is written as:

$$
\text { (B): } \quad y=a x+b
$$

or

$$
\text { (B): } \quad a x-y+b=0 \quad(12)
$$

According to the axis convention of fig. 7, it is easy to argue that:

$$
\text { (13) }\left\{\begin{array}{l}
a=-1 \\
b=\frac{L_{2}}{2}
\end{array}\right.
$$

The problem formation will be established using the symbols $a$ and $b$. The numerical values are used in the case of study (sect. 3). 
The modeling procedure of the distances $\overline{C H}_{i}$ begins with the decomposition of the first quadrant related to the left upper corner as shown the fig. 10. Two horizontal axis are represented on this figure:

- $\quad$ the first (upper) horizontal axis describes the true abscissa $x$ (control abscissa);

- $\quad$ the second (lowest) horizontal axis describes the index abscissa $k$.

The scanning control will be ensured primarily by the index $k$. Reaching the limit $k_{A_{C}}$ of $k$ (limit in the region A), the printing trajectory will is to control according the abscissa $x$ (which takes a limit value $x_{A}$ in the limit of region A). This procedure permitted to elaborate the mathematical developments that are detailed in the appendix.

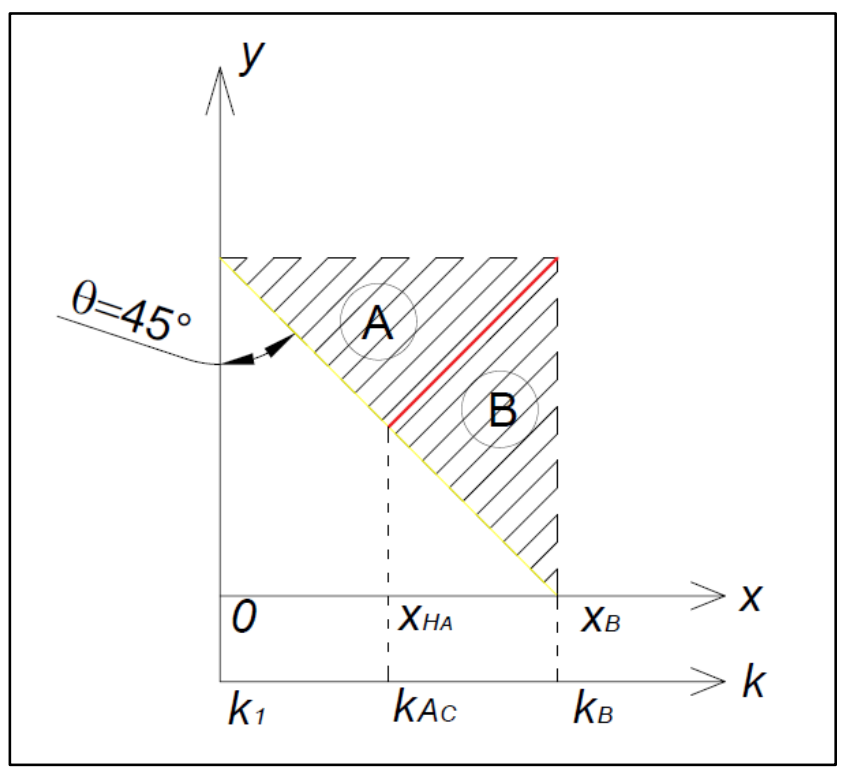

Fig 10 Decomposition of the first quadrant related to the left upper corner of the rectangle

\subsubsection{Productive and non-Productive Lengths of area (A)}

\section{Productive length formulation}

According to the results of the equation (74) of the appendix, the productive length of the area (A) is expressed by the expression (14).

$$
L_{P_{A}}=-a \sqrt{1+a^{2}}\left[\frac{\beta}{2} e_{S} k_{A_{C}}\left(k_{A_{C}}+1\right)+x_{H_{A}}\right](14) \Leftrightarrow(74)
$$

Where, as presented in the fig. 10:

$k_{A_{C}}$ is the correction of the value of the limit of the index $k_{A}$ in the area (A);

$x_{H_{A}}$ is the abscissa of the point $\mathrm{H}$ at the limit of the area (A);

$$
\left\{\begin{array}{c}
k_{A_{C}}=E\left(\frac{\sqrt{2} \alpha_{1} L_{1}}{\left(a^{2}+1\right) e_{S}}\right) \\
x_{H_{A}}=\frac{\alpha_{1} L_{1}}{1+a^{2}}
\end{array}\right.
$$

The equations (15) and (16) correspond resp. to the equations (72) and (73) of the appendix.

\section{Non-Productive length formulation}


According to the results of the equations (75) and (76) of the appendix, the non-productive length of the area (A) is expressed by the system the expressions (17.1) and (17.2).

- If $k_{A_{C}}$ is an impair:

$$
L_{N P_{A}}=E\left(k_{A_{C}} / 2\right)\left(1+\frac{\sqrt{2}}{2}\right) e_{S}
$$

- If $k_{A_{C}}$ is pair:

$$
L_{N P_{A}}=\left(k_{A_{C}}\left(\frac{1+\sqrt{2}}{2}\right)-1\right) e_{S}
$$

\subsubsection{Productive and non-Productive Lengths of the area (B)}

As stated in the sect. B of the appendix, the areas (B) and (A) are symmetrical according the red line drawn in fig. 10. Hence, the productive length $L_{P_{B}}$ and non-productive length $L_{N P_{B}}$ are expressed by the system (18).

$$
\text { (18) }\left\{\begin{array}{c}
L_{P_{B}}=L_{P_{A}} \\
L_{N P_{B}}=L_{N P_{A}}
\end{array}\right.
$$

\subsubsection{Productive and non-Productive Lengths of the area (C)}

As proved in the sect. $\mathrm{C}$ of the appendix, the productive and non-productive lengths of the region $(\mathrm{C})$ are computed, resp. $L_{P_{C}}$ and $L_{N P_{C}}$, using the following expressions (19-21). The equations (19) and (20) are resp. equal to equations (77) and (78) of the appendix.

$$
\begin{aligned}
& L_{P_{C}}=\frac{N_{1 C} L_{2}}{4}\left(\frac{1}{n_{C_{x}}}\left(\frac{L_{2}}{2 n_{C_{x}} n_{C_{y}} e_{s}}+1\right)+\frac{1}{n_{C_{y}}}\left(\frac{\left(L_{1}-L_{2}\right)}{2 n_{C_{x}} e_{s}}+1\right)\right) \\
& L_{N P_{C}}=\frac{L_{2}}{2}\left(\frac{N_{1 C}}{n_{C_{y}}}+\frac{N_{2 C} L_{2}}{2 n_{C_{x}}}\right)(20) \\
& \text { (21) }\left\{\begin{aligned}
& \text { if }\left(\left(n_{C_{x}} \text { is pair }\right) \text { or }\left(n_{C_{y}} \text { is pair }\right)\right): N_{1 C}=N_{2 C}=\frac{n_{C_{x}} n_{C_{y}}}{2} \\
& \text { else }\left\{\begin{array}{c}
\text { if chess begins with pattern } 1: N_{1 C}=E\left(\frac{n_{C_{x}} n_{C_{y}}}{2}\right)+1 \\
\text { if chess begins with pattern } 2: N_{2 C}=E\left(\frac{n_{C_{x}} n_{C_{y}}}{2}\right)+1 \\
N_{1}+N_{2}=n_{C_{x}} n_{C_{y}}
\end{array}\right.
\end{aligned}\right.
\end{aligned}
$$

Where

$N_{l C}$ : is the total number of pattern 1 (fig. 5);

$N_{2 C}$ : is the total number of pattern 2 (fig. 5);

$n_{C_{x}}$ : is the number of divisions of the dimension of the rectangle according to the pattern along the axis $\vec{x}$;

$n_{C_{y}}$ : is the number of divisions of the dimension of the rectangle according to the pattern along the axis $\vec{y}$;

\subsection{Production time of one layer}

The Production time for one layer is composed by active and inactive scanning, expressed in the previous paragraphs by the mean of productive and non-productive lengths. The two following paragraphs present the expressions of the active and non-active production times (resp. lengths) whose summation corresponds to the total layer production time (resp. total production length). 


\subsubsection{Total active production time}

The scanning is activated in between to respective jumps. For example, this corresponds to laser spot activation or extrusion filament feeding, etc. As written in the previous paragraphs, the active lengths were denoted $L_{P_{A}}$ and $L_{P_{C}}$ respectively for the areas $(\mathrm{A})$ and $(\mathrm{C})$.

According to fig. 7, the area $(A \cup B)$ is repeated eight time, two by two at each corner of the rectangle; the area (C) is studied as a sole compartment.

So, the expression of the active production length of one layer is given by:

Since

$$
L_{P}=8 L_{P_{A}}+8 L_{B}+L_{P_{C}}
$$

Thus

$$
L_{P_{B}}=L_{P_{A}}
$$

$$
L_{P}=16 L_{P_{A}}+L_{P_{C}}
$$

Considering a constant active scanning speed $V_{P}$, the total active production time is given by the expression (23).

$$
T_{P}=\frac{L_{P}}{V_{P}}
$$

\subsubsection{Total inactive production time}

The inactive production scanning speed is denoted $V_{N P}$. Similarly, as stated for the total active length, the total non-active length is expressed by the equation (24).

$$
L_{N P}=16 L_{N P_{A}}+L_{N P_{C}}
$$

Considering a constant inactive production speed $V_{N P}$, the total non-productive time is given by the expression (25).

$$
T_{N P}=\frac{L_{N P}}{V_{N P}}
$$

\subsubsection{Total production time}

For the proposed SBP scanning strategy, the total production time $T_{S B P}$ of one layer of the rectangle is computed using the expression (26).

$$
T_{S B P}=T_{P}+T_{N P}
$$

Or

$$
T_{S B P}=\frac{L_{P}}{V_{P}}+\frac{L_{N P}}{V_{N P}}
$$

In the case of equal active and inactive scanning speeds $\left(V_{P}=V_{N P}\right)$, the equation (26) becomes:

$$
T_{S B P}=\frac{1}{V_{P}}\left(L_{P}+L_{N P}\right)
$$

That is expressed as:

$$
T_{S B P}=\frac{L_{S B P}}{V_{P}}
$$


Where $L_{S B P}$ is the total run length, productive and non-productive.

$$
L_{S B P}=L_{P}+L_{N P}(29)
$$

\subsection{Gain in time and energy}

2.5.1. Specific Gain: gain of production time per surface unit

The gain per surface unit was computed by considering a series of rectangles $\left(L_{1} \times L_{2}\right)$. For each hatch space distance, and according to $n_{C_{x}}$ and $n_{C_{y}}$, the maximal gains, are computed and divided by the area $\left(L_{1} \times L_{2}\right)$ of the corresponding rectangle. For each hatch space, this measure corresponds to the maximal gain of production time per surface unit.

The gain of production time is computed by the expression (30) and the specific gain per surface unit is computed by the expression (31).

$$
\operatorname{Gain}[\%]=\frac{T_{S B P}-T_{c h}}{T_{c h}} \times 100
$$

For each $\left(L_{1} \times L_{2}\right)$ rectangle:

$$
S G\left[\% / m m^{2}\right]=\frac{\text { Gain }}{L_{1} \times L_{2}}
$$

It is clear that SG expresses the gain of time per surface. It can be considered as an indicator of scanning performance for a given area of surface while two or more scanning strategies are compared.

Consequently, according to the expression (31), the SG index is then a function of:

- The arguments of $T_{S B P}$ function that are $n_{C_{x}}, n_{C_{y}}, e$;

- The arguments of $T_{c h}$ function $n_{x}, n_{y}, e$;

- $\quad$ The rectangle dimensions $L_{1}$ and $L_{2}$;

So it is possible to express this dependency as:

$$
S G=S G\left(n_{C_{x}}, n_{C_{y}}, n_{x}, n_{y}, e, L_{1}, L_{2}\right)
$$

Given the above, a total visualization of the SG function is difficult to exhibit graphically. Hence, the most important projections are presented in the results section using the following procedure:

- Variables to fix:

- $n_{x}$ and $n_{y}$ : it means that the chess scanning strategy for all $\left(L_{1} \times L_{2}\right)$ rectangles are fixed;

- $n_{C_{x}}, n_{C_{y}}$ : after computing SG function with the prefixed $n_{x}$ and $n_{y}$ variables, SG was projected on a subspace that maximizes SG according to $n_{C_{x}}$ and $n_{C_{y}}$;

- Variables to maintain (to vary): $e, L_{1}, L_{2}$

This procedure led to the analysis of a maximal specific gains $\mathrm{SG}^{*}$ according to $n_{C_{x}}$ and $n_{C_{y}}$ variables with fixed $n_{x}$ and $n_{y}$. So, the mathematical formulation of $\mathrm{SG}^{*}$ can be written by the equation (33).

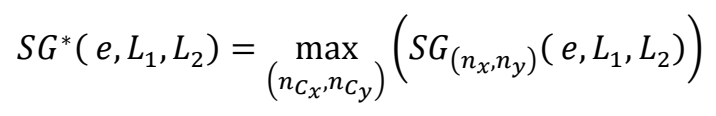

$S G^{*}$ expresses the maximal specific gain per surface unit; 
From the equations (30) to (31), one can argue that three possible scenarios could emerge as simulation results:

- $\quad S G, S G^{*}<0:$ SBP strategy is more competitive than the chess strategy

- $\quad S G, S G^{*}>0$ : chess strategy is more competitive than the SBP strategy

- $S G, S G^{*}=0:$ the two strategies are similar in total production time

\subsubsection{Gain of energy}

Considering the initial formula of the gain of time (30), the denominator and denominators were multiplied by the laser power P:

$$
\operatorname{Gain}[\%]=\frac{\left(T_{S B P}-T_{c h}\right) P}{T_{c h} P} \times 100=\frac{\left(E_{S B P}-E_{c h}\right)}{E_{c h}} \times 100
$$

So

$$
\operatorname{Gain}[\%]=-\left(1-\frac{E_{S B P}}{E_{c h}}\right) \times 100
$$

Where

$$
\left\{\begin{array}{l}
E_{S B P}: \text { energy consumption related to } S B P \text { scanning strategy } \\
E_{c h}: \text { energy consumption related to chess scanning strategy }
\end{array}\right.
$$

Hence, one can see that the modified formula of the gain of time corresponds also to a gain of energy. Consequently the specific gains $S G$ or the maximal specific gain per surface unit $S G^{*}$ resp. express the gain of energy and the maximal specific gain of energy per surface unit.

\subsection{Cases of study descriptions}

\subsubsection{Case of study 1}

Given a rectangle with a fixed dimensions $\left(L_{1}, L_{2}\right)_{L_{1}>L_{2}}$, the first case of study is dedicated to compare :

- $\quad$ a given configuration of a pre-known Chess strategy that can be expressed by a fixed couple $\left(n_{x}, n_{y}\right)$;

- $\quad$ a series of SBP strategies that can are described by variable couples $\left(n_{C_{x}}, n_{C_{y}}\right)$ that are considered as problem variables;

In this problem, the hatch distance of the two strategies in competition are equal, and are varied according to the bibliography presented in the table 1 of sect. 1 . The scanning speed is also fixed. Table 2 groups the input parameters used in the case of study 1 .

Table 2 Geometrical and scanning parameters used in the case of study 1

\begin{tabular}{|l|c|}
\hline \multicolumn{1}{|c|}{ Parameters } & Values \\
\hline \multirow{2}{*}{ Geometry of the rectangle } & $L_{1}=100 \mathrm{~mm}$ \\
\cline { 2 - 2 } Chess strategy decomposition & $L_{2}=50 \mathrm{~mm}$ \\
\hline \multirow{3}{*}{ Limit conditions for SBP strategy } & $n_{x}=100$ \\
\cline { 2 - 2 } & $n_{y}=50$ \\
\cline { 2 - 2 } & $\min \left(n_{C_{x}}\right)=2$ \\
\cline { 2 - 2 } & $\max \left(n_{C_{x}}\right)=30$ \\
\cline { 2 - 2 } & $\min \left(n_{C_{y}}\right)=2$ \\
\cline { 2 - 2 } & $\max \left(n_{C_{x}}\right)=30$ \\
\hline
\end{tabular}




\begin{tabular}{|c|c|}
\hline \multirow{2}{*}{ Hatch limit conditions ${ }^{(*)}$} & hatch $_{\min }=e_{\text {res }}=25 \mu \mathrm{m}$ \\
\hline & hatch $_{\max }=e_{c}=1 \mathrm{~mm}$ \\
\hline Scanning speed ${ }^{(*)}$ & $V_{p}=800 \mathrm{~mm} / \mathrm{s}$ \\
\hline
\end{tabular}

\subsubsection{Case of study 2: Maximal Specific Gain per Surface Unit (SG*)}

In order to analyze the proposed indictor $\mathrm{SG}^{*}$, a set of $\left(L_{1}, L_{2}\right)_{L_{1}>L_{2}}$ rectangles and the corresponding areas $A$ were generated by the following procedure:

$$
\text { (36) }\left\{\begin{array}{l}
\forall i, j \in\{1, \ldots, 20\} \\
\left\{\begin{array}{l}
L_{1}(i)=100+10 \times i \\
L_{2}(j)=50+10 \times j
\end{array}\right. \\
\left\{\begin{array}{l}
\text { if } L_{1}(i)>L_{2}(j): A(i, j)=L_{1}(i) \times L_{2}(j) \\
\text { else } A(i, j)=N a N
\end{array}\right.
\end{array}\right.
$$

The other scanning parameters are chosen according to the table 2 .

\subsection{Gain of time and specific gain if time Surface modeling}

After computation the gain of time function and the maximal specific gain of time $S G^{*}$, both were modeled according to linear (refer to the discussion sect. 4.2). For these models, the gain of time and $S G^{*}$ surfaces are modeled according to $L_{1}$, and $L_{2}$; the hatch distance e is treated as a model parameter instead a modeling variable; but it will be involved in the modeling of the parameters of the regressive models $\left(b_{0}, b_{1}, b_{2}\right)$ as presented by the equation (37).

$$
\operatorname{gain}_{e}=b_{0}(e)+b_{1}(e) L_{1}+b_{2}(e) L_{2}
$$

A more detailed modeling of $S G_{e}^{*}$ is proposed according to the following:

$$
\begin{array}{r}
\forall\left(L_{1}, L_{2}\right)_{L_{1}>L_{2}} \in[100,300] \times[50,250] ; \\
S G_{e}^{*}\left(L_{1}, L_{2}\right)=b_{0}(e)+b_{1}(e) L_{1}+b_{2}(e) L_{2} \\
\text { (39) }\left\{\begin{array}{l}
b_{0}(e)=\widetilde{S G}_{e}^{*}(0,0)=\alpha_{0}+\beta_{0} e \\
b_{1}(e)=\frac{\partial \widetilde{S G}_{e}^{*}\left(L_{1}, L_{2}\right)}{\partial L_{1}}=\alpha_{1}+\beta_{1} e \\
b_{2}(e)=\frac{\partial \widetilde{S G}_{e}^{*}\left(L_{1}, L_{2}\right)}{\partial L_{2}}=\alpha_{2}+\beta_{2} e
\end{array}\right.
\end{array}
$$

\section{Optimization Problem formulation}

\subsection{Objective function}

This section presents the formulation of the optimization problem related to the minimization of the total production time of a given rectangular layer by the use of the SBP technique. At first, the model will take into account all the possible parameters in term of geometry parameters, printing, and jump speeds. In summary, six independent decision variables are included in the analysis:

- $\quad e$ : chess hatch space;

- $\quad\left(n_{x}, n_{y}\right)$ : resp. the divisions according to $\vec{x}$ and $\vec{y}$ directions of the rectangle related to the chess strategy;

- $\quad e_{s}$ : skeleton hatch space;

- $\quad\left(n_{C x}, n_{C y}\right):$ resp. the divisions of the area $C$ related to the SBP technique;

The function to minimize corresponds to the total production time related to SBP strategy. It is then expressed as: 


$$
f\left(e_{s}, n_{C x}, n_{C y}\right)=T_{S B P}\left(e_{s}, n_{C x}, n_{C y}\right)
$$

\subsection{Problem constraining}

In order to ensure the competitiveness of the SBP regarding the chess scanning strategy, the total production time production of the SBP strategy $T_{S B P}$ must be less than the classical chess strategy production time $T_{c h}$. This condition is expressed by the inequality (41).

$$
T_{S B P} \leq T_{c h}
$$

If we consider equal scanning speeds for the two strategies in competition, the equation (42) becomes:

$$
L_{S B P} \leq L_{c h}
$$

From a technical stand-point, the hatch distance of both SBP and chess strategies must be greater than or equal to the printer scanning resolution $e_{r e s}$ and less than a limit value $e_{c}$, as discussed in the sect. 1. These two conditions are expressed by the inequalities (43) and (44).

$$
\left\{\begin{array}{l}
e_{\text {res }} \leq e_{s} \leq e_{c} \\
e_{\text {res }} \leq e \leq e_{c}
\end{array}\right.
$$

\subsection{Optimization Problem formulation}

The optimization problem to adopt is proposed as follows:

- Considering a fixed rectangle $\left(L_{1}, L_{2}\right)$;

- Considering a chess scanning strategy of the rectangle that is characterized by prefixed $n_{x} \times n_{y}$ divisions;

- The hatch distance $e$ of chess strategy can be varied, and it is considered as a parameter in the optimization problem.

Now, the generalized Optimization Problem $\left(\mathrm{OP}_{1}\right)$ can be formulated as follows:

For a given rectangle $\left(L_{1}, L_{2}\right)$ and $\left(n_{x}, n_{y}\right)$ chess scanning strategy:

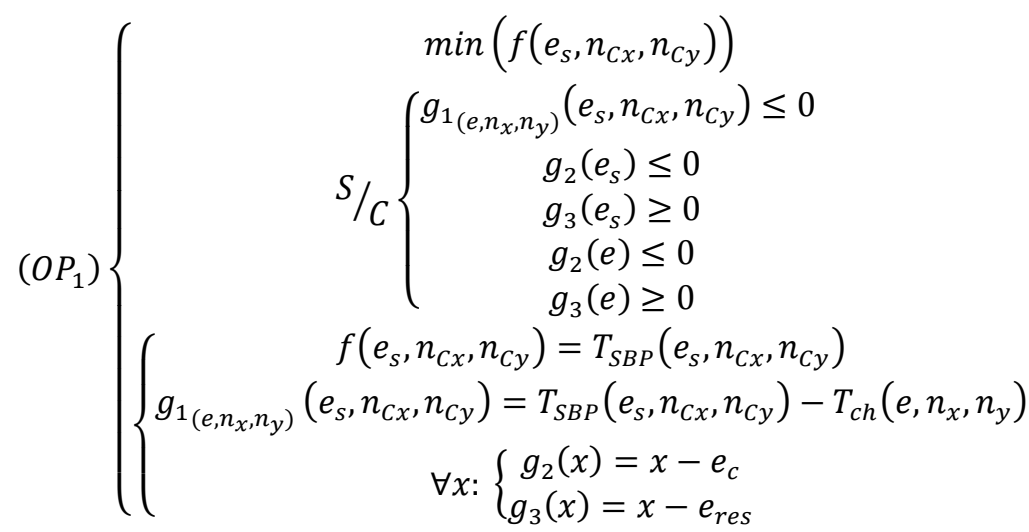

In order to minimize the probable mechanical and metallurgical characteristics differences between the resulting parts of the two strategies in competition, the hatch distances $e$ (chess) and $e_{s}$ (skeleton) are forced to be equal $\left(e=e_{s}\right)$. This new constraint leads to a simplified optimization sub-problem $\left(O P_{2}\right)$ as follows:

For a given rectangle $\left(L_{1}, L_{2}\right)$ and $\left(n_{x}, n_{y}\right)$ chess scanning strategy 


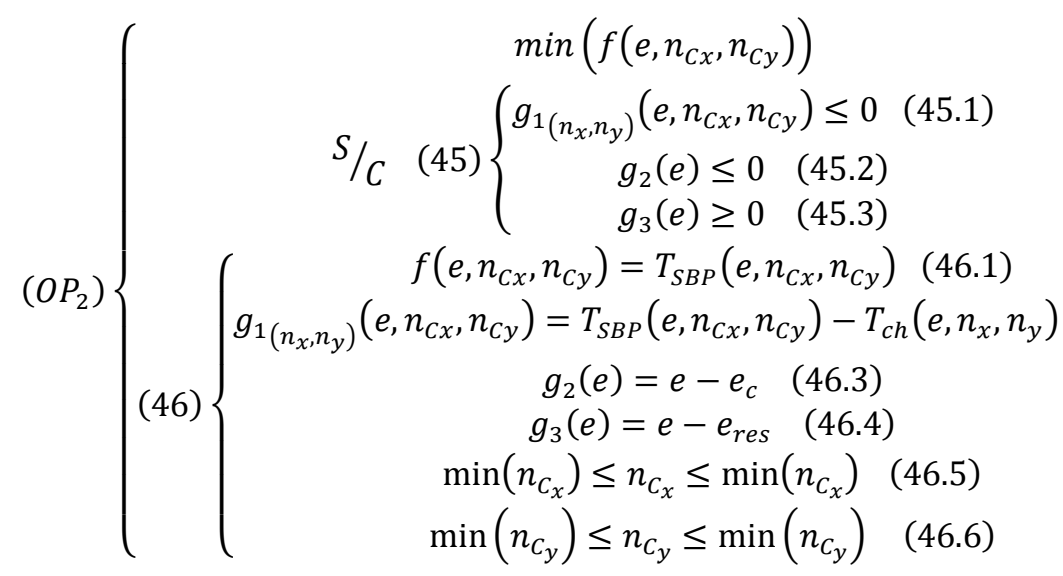

\section{Results and discussion}

\subsection{Case of study 1}

As explicated in the constraint (45.1) of the sub-optimization problem $\left(\mathrm{OP}_{2}\right)$, it is clear that the minimization of the objective function depends on the competitive chess strategy that I in competition with the proposed SBP strategy. That is to say that the proposed approach takes it start by fixing a given chess strategy that is taken as the major problem constraint. Hence, for each couple \{rectangle $\left(L_{1} \times L_{2}\right)$, chess strategy $\left.\left(n_{x}, n_{y}\right)\right\}$, the resolution will generate different solutions. In addition, the hatch space limits $\left(e_{r e s}, e_{c}\right)$ are necessary to restrict the analysis on a reasonable range of hatch spacing.

So, all parameters of this case of study are as described in table 2 of sect. 2.6.1.

\subsubsection{Objective function evaluation}

The objective function corresponds to the expression (46.1) of the $\left(O P_{2}\right)$. The plots of fig. 11 present display the objective function as a family of surfaces $T_{S B P}\left(f, n_{C x}, n_{C y}\right)$ parametrized by the hatch distance e. The representation space corresponds to the 3D-space $\left(f, n_{C x}, n_{C y}\right)$. Moreover, the intercept of the surfaces and the variations according to $n_{C_{x}}$ and $n_{C_{y}}$ were computed in order to estimate the evolution of the total production time according to these decision variables.

$T_{S B P}$ surfaces present discontinued zones that are generated by the existence of integer parts of some subexpression of the general formula. That is why a general tendency of the first order behavior of $T_{S B P}$ surfaces is proposed. The estimation of the intercept and the slopes of $T_{S B P}$ surfaces was estimated using surface linear regression. The regression models of the $T_{S B P}$ surfaces are not too significant since the coefficient of determination $\mathrm{R}^{2}$ are between $10 \%$ and $35 \%$, but a general first order tendency could be detected from this preliminary analysis and the regression models are no longer discussed in the next developments.

Fig. 12.a portrays the superposition of the surfaces $T_{S B P}\left(n_{C x}, n_{C y}, e\right)$ in the same space. Fig. 12.b-12.c are depicted resp. as follows:

- the estimated intercepts of the surfaces $T_{S B P}: b_{0}=\widetilde{T}_{S B P}(0,0, e)$

- the estimation of the first order variation of the surfaces $T_{S B P}$ according to $n_{C_{x}}: b_{1}=\partial \widetilde{T}_{S B P} / \partial n_{C_{X}}$

- the estimation of the first order variation of the surfaces $T_{S B P}$ according to $n_{C_{y}}: b_{2}=\partial \widetilde{T}_{S B P} / \partial n_{C_{x}}$ where $\tilde{T}$ is the estimated family of surfaces at each hatch space value. 


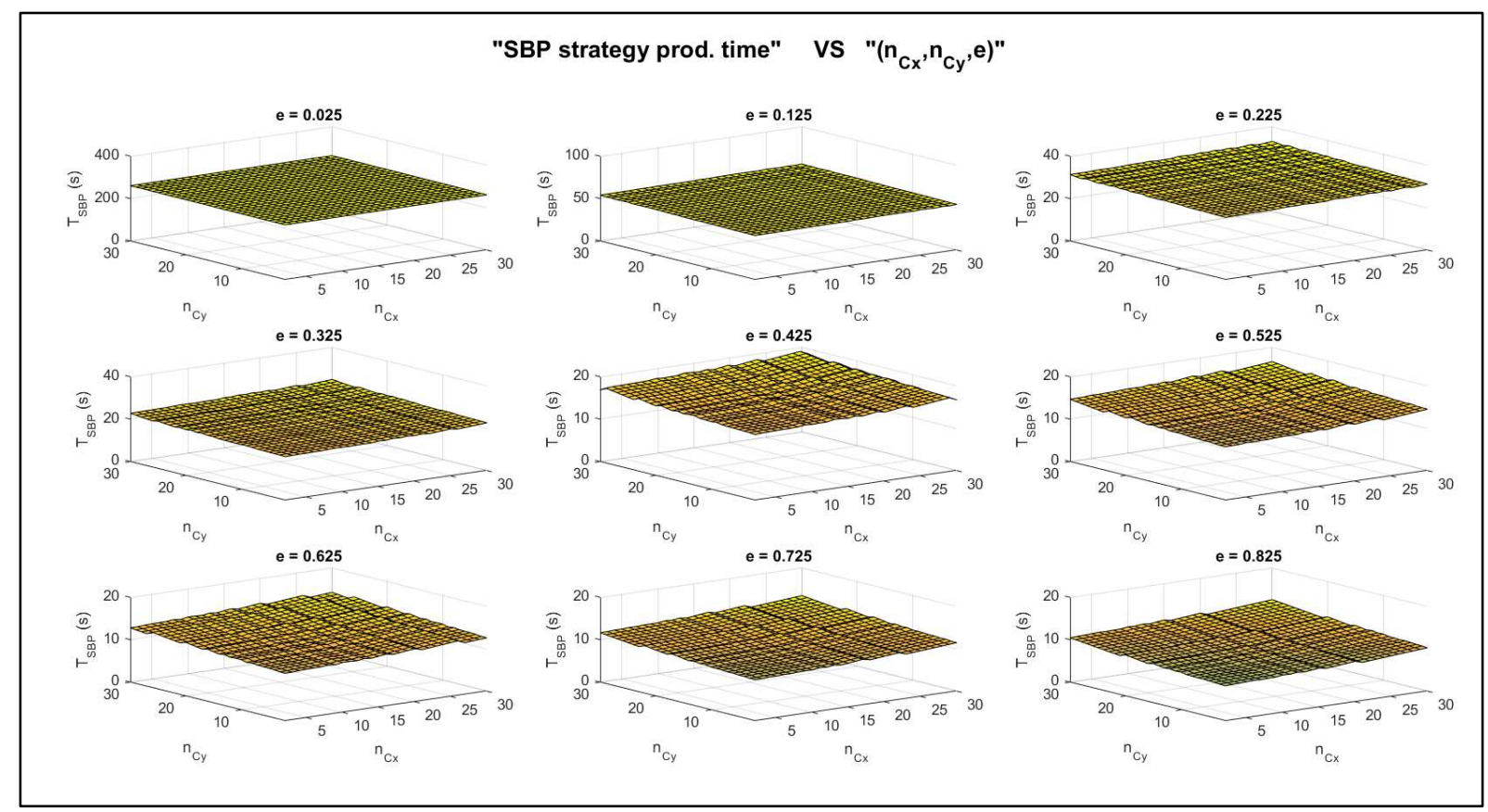

Fig 11 Production time of the SBP strategy according to $\left(n_{C x}, n_{C y}, e\right)$

From fig. 12.a-12.e, one can observe that:

1) the parameters $\left(b_{0}, b_{1}, b_{2}\right)$ are positive, especially $b_{1}$ and $b_{2}$ which means that the production time $\widetilde{T}_{S B P}$ increases according to the division variables $n_{C_{x}}$ and $n_{C_{y}}$ that describe the $\mathrm{C}$ area scanning strategy;

2) the evolution of the parameters $\left(b_{0}, b_{1}, b_{2}\right)$ highly depends on hatch spacing and are exponentially decreasing according to it. $\left(b_{0}, b_{1}, b_{2}\right)$ were fitted according to the hatch space $e$ using exponential model (fig. 12.c-12.e); the coefficient of determination $\mathrm{R}^{2}$ is higher than $75 \%$ which means that the fitting is good;

3) a full linear correlation between the parameters $\left(b_{0}, b_{1}, b_{2}\right)$ can be observed from the (fig. 12.b).

Hence, it is possible to recapitulate the evolution of the production time $T_{S B P}$ as follows:

- $T_{S B P}$ increases quasi-linearly according to the decomposition parameters $\left(n_{C x}, n_{C y}\right)$ of the $\mathrm{C}$ area;

- $\quad T_{S B P}$ decreases exponentially according to the hatch distance $e$. 


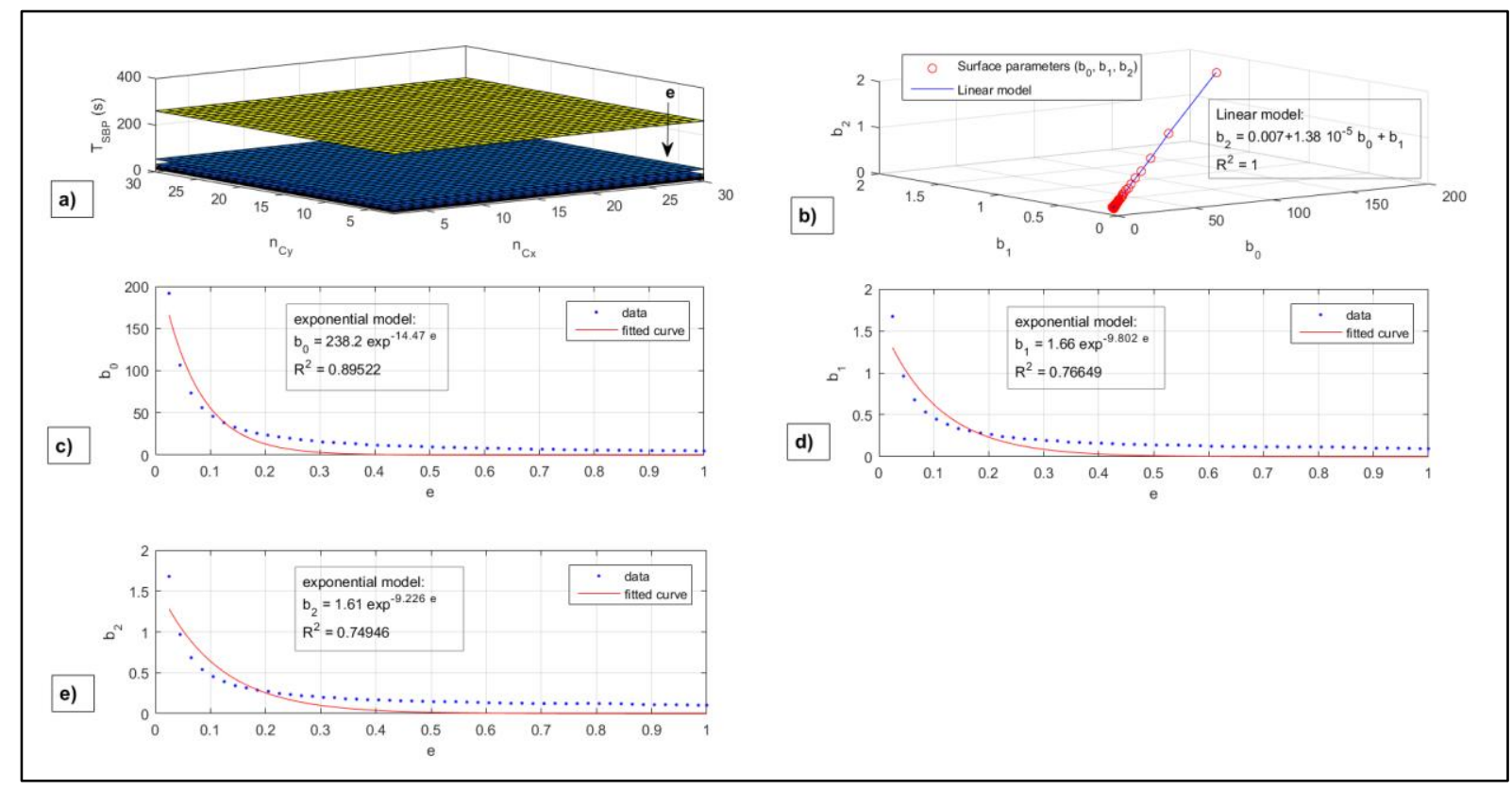

Fig. 12 Variation of the $T_{S B P}$ surfaces parameters a) Superposition of the surfaces family $T_{S B P}$ b) 3D-plot of $\left(b_{0}, b_{1}, b_{2}\right)$ c) Evolution of the intercepts $b_{0}$ according to hatch space e d) Evolution of first order variation $b_{1}$ according to hatch space e e) Evolution of first order variation $b_{2}$ according to hatch space e

These remarks, that are summarized in the table 3, permitted to appreciate the effect of the decision variables $\left(n_{C x}, n_{C y}, e\right)$ on the production time $T_{S B P}$ for the ultimate objective which is to resolve the $\left(O P_{2}\right)$ optimization problem by minimizing the production time printing.

Table 3 Approached variations signs of $\widetilde{T}_{S B P}$ surfaces

\begin{tabular}{|c|c|c|}
\hline Variations ${ }^{(*)}$ & Sign & Behavior \\
\hline$\frac{\partial \tilde{T}_{S B P}}{\partial e}$ & - & exponentially \\
\hline$b_{0}(e)=\tilde{T}_{S B P}(0,0, e)$ & - & exponentially \\
\hline$b_{1}=\frac{\partial \tilde{T}_{S B P}}{\partial n_{x}}$ & + & little slope \\
\hline$b_{2}=\frac{\partial \tilde{T}_{S B P}}{\partial n_{y}}$ & - & little slope \\
\hline$\frac{\partial b_{0}}{\partial e}={ }^{\partial \tilde{T}_{S B P}(0,0, e) / \partial e}$ & - & exponentially \\
\hline$\frac{\partial b_{1}}{\partial e}={ }^{\partial\left(\frac{\partial \tilde{T}_{S B P}}{\partial n_{x}}\right) / \partial e}$ & - & exponentially \\
\hline$\frac{\partial b_{2}}{\partial e}={ }^{2}\left(\frac{\partial \tilde{T}_{S B P}}{\partial n_{y}}\right) / \partial e$ & - & exponentially \\
\hline
\end{tabular}

\subsubsection{Feasible space evaluation}

The feasible space is computed according to the inequalities (45.1-45.3) and the expressions (46.2-46.6) of the sub-optimization problem $\left(\mathrm{OP}_{2}\right)$. As introduced previously, the SBP and chess strategies are compared at an equivalent hatch space. Then hatch space is then varied as presented in the $\left(\mathrm{OP}_{2}\right)$ problem definition (equations 46.3-46.4 and expressions 45.2-45.3).

Fig. 13 presents the plot of the $g_{1}$ surfaces at different hatch space values. It is necessary to recall that $g_{1}$ function expresses the difference between the production times of the SBP and the chess strategies. 


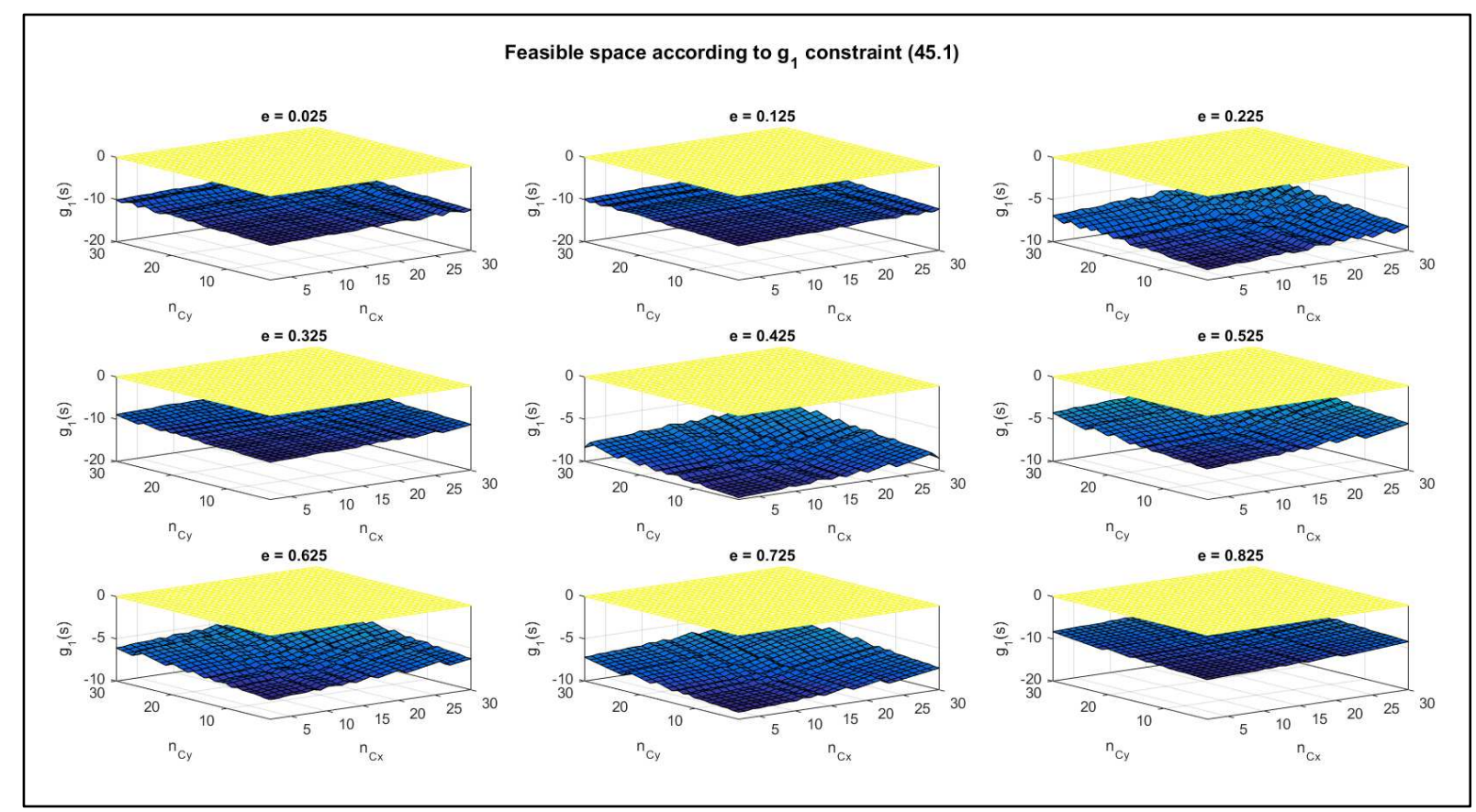

Fig. $13 g_{1}$ function values according to $\left(n_{C x}, n_{C y}, e\right)$

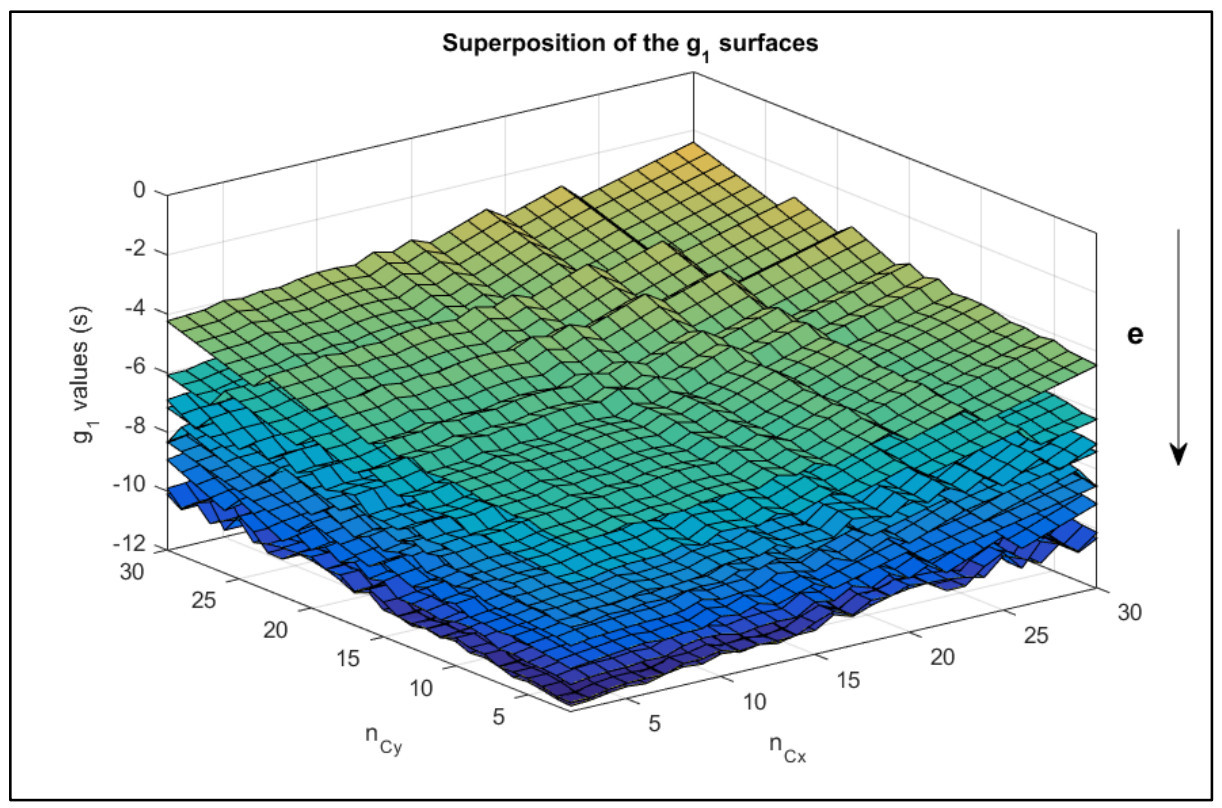

Fig. 14 Superposition of the $g_{1}$ surfaces according to $\left(n_{C x}, n_{C y}, e\right)$

Based on the plots of fig. 13 and 14 , it is noticeable that $g_{1}$ is totally negative, which means that, for this case of study, the feasible space is covered by all the decision variables configurations. Typically, the cubic domain D expressed by the $\mathrm{R}^{3}$ sub-space (47) in term of $\left(n_{C_{x}}, n_{C_{y}}\right)$ and e parameters.

$$
D=[2,30] \times[2,30] \times[25 \mu \mathrm{m}, 1 \mathrm{~mm}]
$$

\subsubsection{Performance and resolution discussion of $\left(\mathrm{OP}_{2}\right)$}

Since the feasible space corresponds to the Cartesian product described by the expression (47), the minimization of the problem is discussed on the whole decision variables intervals.

According to the computation results, the $T_{S B P}$ function present a general tendency to increasing: 
- $\quad$ starting from the initial point $\left(n_{C x}, n_{C y}, e\right)=(2,2,25 \mu m)$ :

- $\quad " T_{S B P}=250.82 s "$

- with a production time gain equal to $g_{1}=|-10.31 \mathrm{~s}|$

- with a gain percentage of $\left|\frac{g_{1}}{T_{c h}}\right|=3.08 \%$

- $\quad$ starting from the initial point $\left(n_{C x}, n_{C y}, e\right)=(30,30,1 \mathrm{~mm})$ :

- $\quad T_{S B P}=9.75 s "$

- with a production time gain equal to $g_{1}=|-0.48 s|$

- with a gain percentage of $\left|\frac{g_{1}}{T_{c h}}\right|=45.51 \%$

Fig. 15 portrays in percentage the maximal time gain according to the hatch space distance. From fig. 15, the gain in term of time of production varies from $3.08 \%$ to $45.51 \%$ according to the value of hatch space.

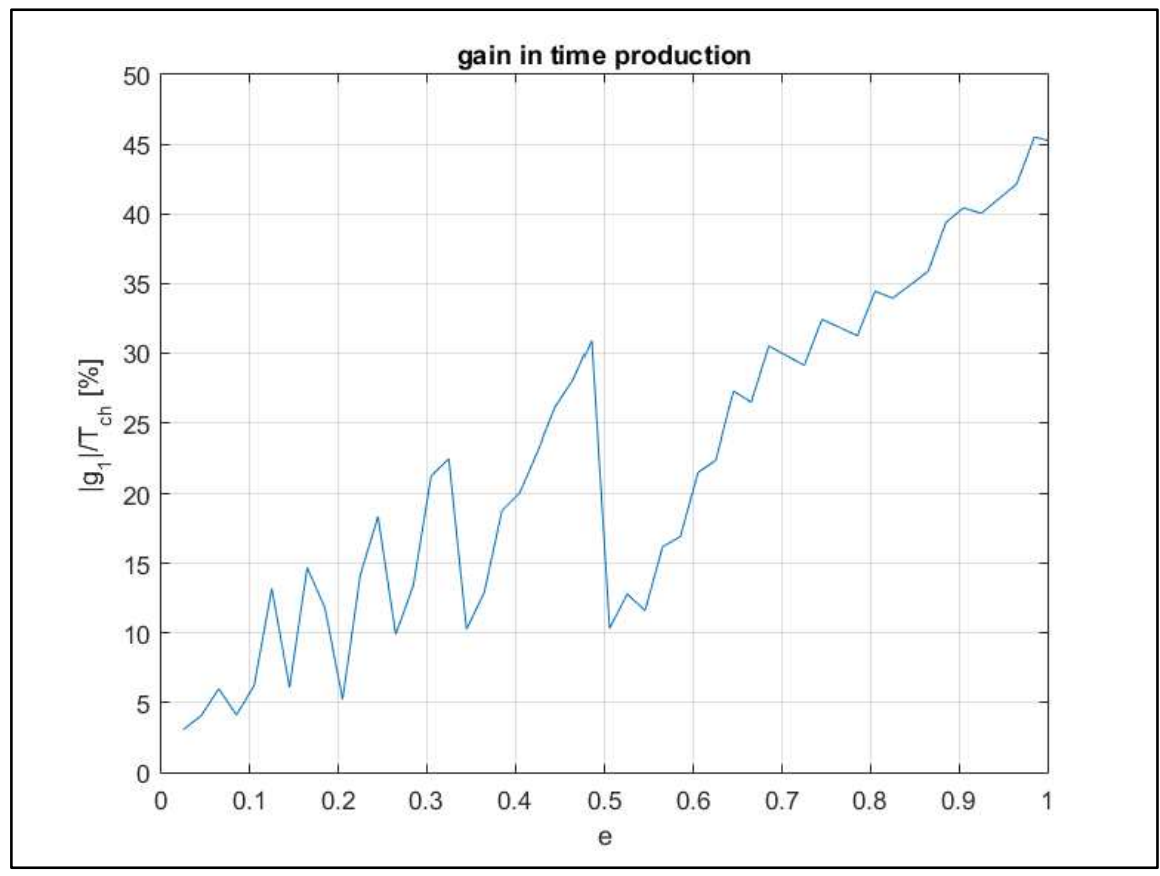

Fig. 15 Gain in term of time of production

According to the variation analysis of table 3 , since the SBP production time presents a general tendency to increase with $n_{C_{x}}$ and $n_{C_{y}}$, the objective function $T_{S B P}$ reaches its minimum for each hatch space $e$ at the lowest values of $\left(n_{C_{x}}, n_{C_{y}}\right)$ as described by the expression (48).

$$
\forall e: \quad \operatorname{argmin}\left(T_{S B P}\left(\mathrm{n}_{C_{\mathrm{x}}}, \mathrm{n}_{\mathrm{C}_{\mathrm{x}}}\right)\right)=\left(\min n_{C_{x}}, \min n_{C_{y}}\right)
$$

Thus

$$
\forall e: \quad \operatorname{argmin}\left(T_{S B P}\left(\mathrm{n}_{\mathrm{C}_{\mathrm{x}}}, \mathrm{n}_{\mathrm{C}_{\mathrm{x}}}\right)\right)=(2,2)
$$

Hence, for this case of study, the discussion according the production performance can be closed as this step.

\subsubsection{Discussion on the solutions}

One can argue that the mathematical solution (48) of the $\left(\mathrm{OP}_{2}\right)$ is not logical from mechanical and physical standpoints. If the $\mathrm{C}$ area is divided into 4 parts according to (49), the dimensions of each pattern will be very important around $\left(\left(a_{C_{x}}, a_{C_{y}}\right)=(25 \mathrm{~mm}, 25 \mathrm{~mm})\right)$ compared to the bibliography. This important dimensions can generate two major kind of defaults dues to thermomechanical effects: 
a) if the support of the layer in-process is not too rigid, important distortion rates could appear on the printed part. This can occur due to a higher course of the laser along the $\mathrm{C}$ pattern unities. The melting/solidification phenomenon is the cause of this process defaults;

b) if the rectangle is well-fixed (clamped) in the printer substrate, distortion will be less, but higher residual stresses could be generated in the parts since the distortion energy is not dissipated and it is stocked in the solid material.

These remarks can be argued by exploiting the flatness deviation (FLT) metric that was proposed by Le Roux et al. (2018). Indeed, the FLT indicator or metric has been used in the metrology of machined surfaces in order to estimate the precision of machining and the quality of the machined parts. From the experimental findings of the same authors, the higher is the course of the laser the greater is the distortion. This remark is not explicated in the comments of Le Roux et al. (2018) but it is possible to conclude by comparing the fig. 10.a to 10.c of article [27]. Furthermore, the same conclusive remarks are proved by Li et al. 2018 that analyzed also different scanning strategies and the related distortions (see figure 4.b and sect. 3.3) [47].

Moreover, Li et al. (2017) presented numerical simulations of residual stress and distortion analysis of a cantilever beam on which, in addition to the classical fixation from one side of the beam, two major different limit conditions were applied [40]:

- bottom surface clamping (with a support on substrate);

- free bottom surface (without a support on substrate);

The analysis of the Li et al. (2017) shows that, for the first configuration (bottom surface clamping), high values of residual stresses were generated on all the part. For the second limit condition (free bottom surface), the residual stresses presented highest values only at the beam clamping region [40].

Given the above, deeper analysis on mechanical and metallurgical considerations will be discussed in future works.

\subsection{Case of study 2: Analysis of Gain of time and gain of time per surface unit}

As it was introduced in sect. 2.6.2, a series of rectangles $\left(L_{1}, L_{2}\right)$ are considered for the case of study 2 . The gain of time reached by the adoption of the new SBP scanning strategy instead the classical chess is now divided by the surface of the corresponding rectangles (refer to eq. (31) of sect. 2.5.1). Hence, the gain is then replaced by a specific gain in percentage per surface unit $\left(\% / \mathrm{mm}^{2}\right)$.

So, the gain of time and gain of time per surface unit were computed according to the procedure of sect. 2.4 and to the input parameters as detailed in section 2.6.2.

\subsubsection{Gain of time}

Fig. 16 displays the gain of time surfaces according to the rectangle dimensions $\left(L_{1}, L_{2}\right)_{L_{1}>L_{2}}$ and to the hatch distance $e$. In these figures, the yellow horizontal plan is the null plan $P_{Z}(Z=0) . P_{Z}$ plan display permits to verify the signs of the computed gains. According to these plots, for all hatch spaces values e and rectangles geometries $\left(L_{1}, L_{2}\right)_{L_{1}>L_{2}}$, it is remarkable that the gain of time function is negative, which mean that the SBP scanning production time is less than chess scanning production time. 

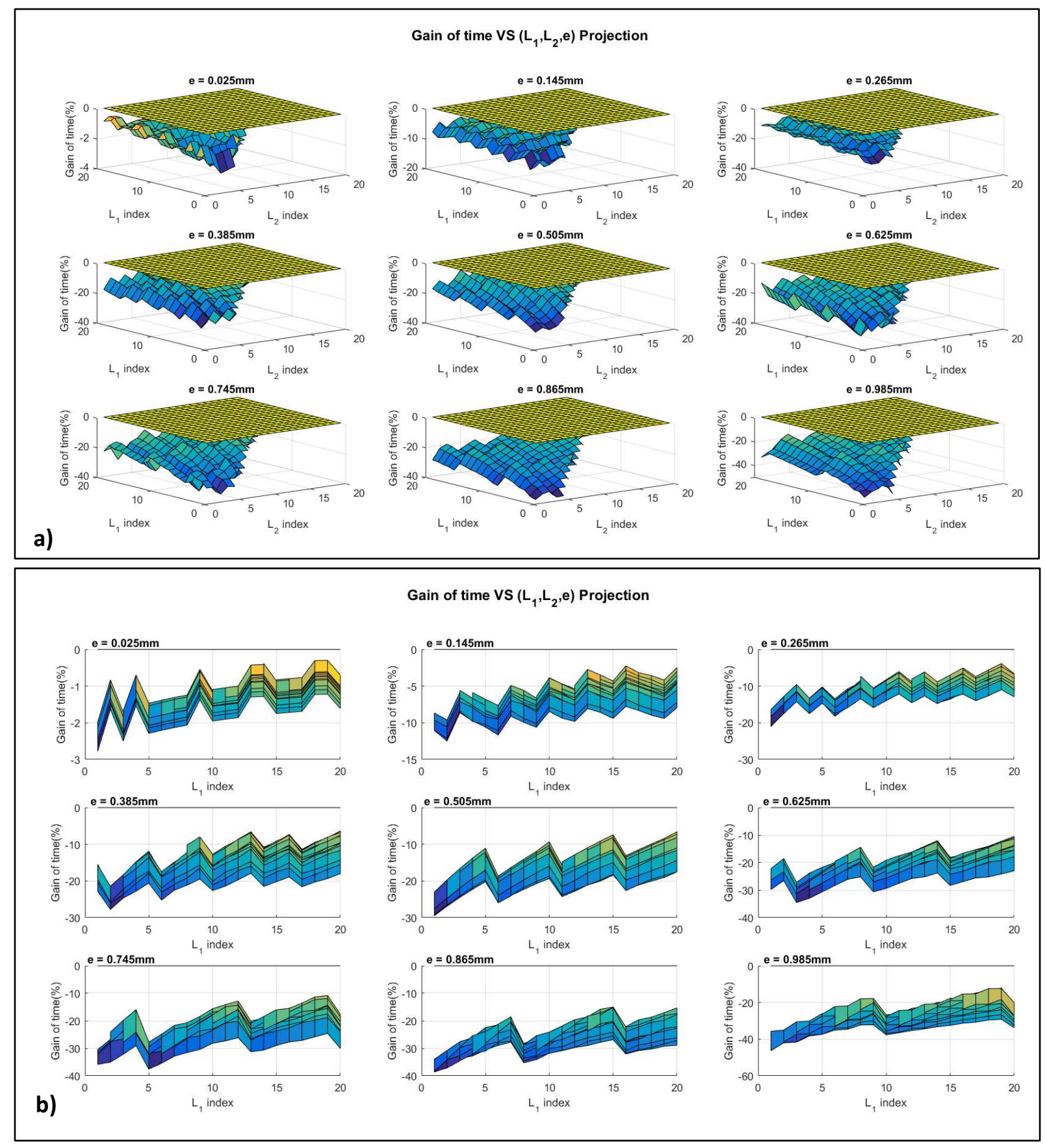


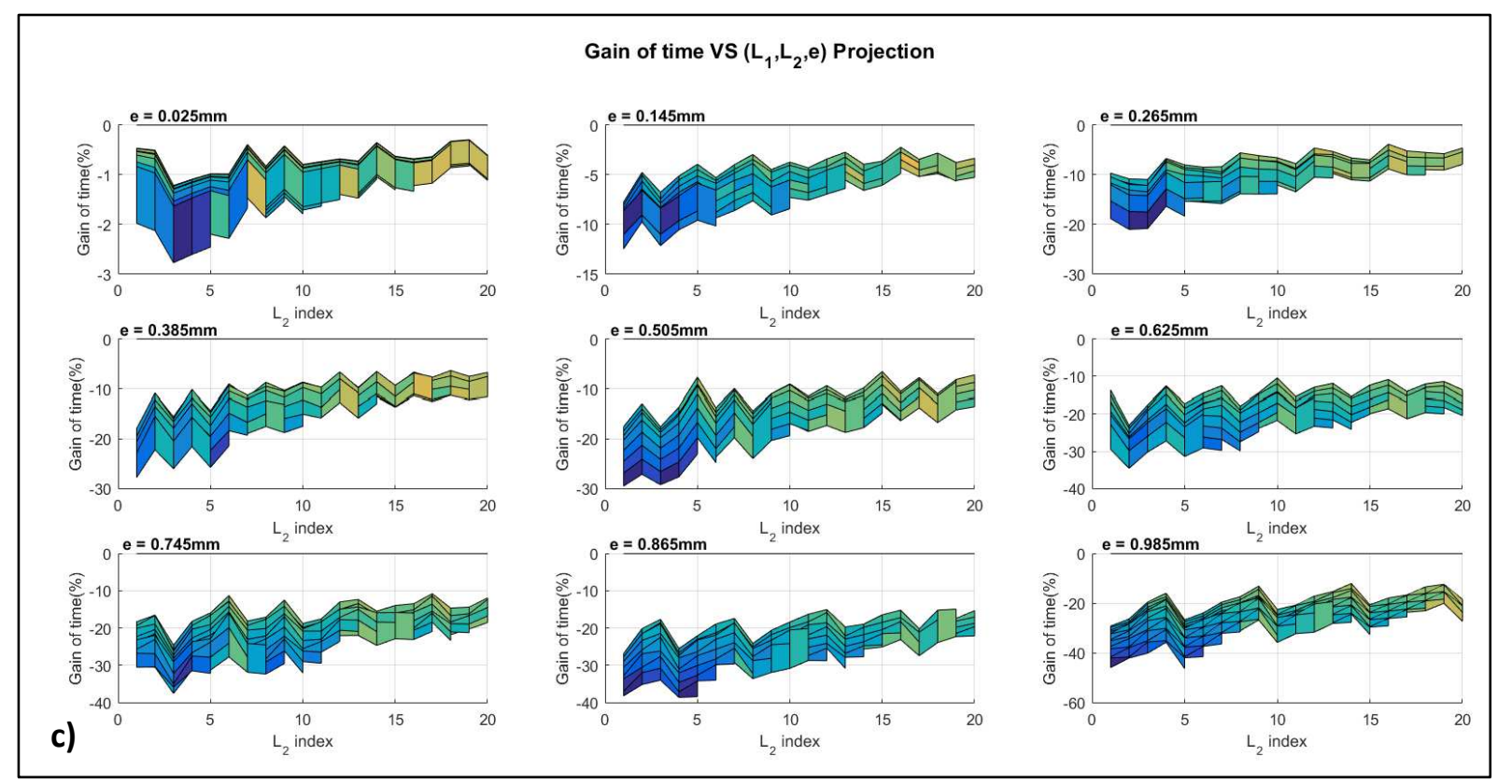

Fig. 16 Gain of time of production according to $\left(L_{1}, L_{2}, e\right)$ parameters a) 3D view b) projection parallel to $L_{2}$ c) projection parallel to $L_{1}$

According to the figures 16.b and 16.c, a global increasing of the gain surface is observed according to $L_{1}$ and $L_{2}$, but local changes of the slopes are emphasized. It could be explained by the existence of the integer parts of some sub-expressions $T_{S B P}$ and $T_{c h}$ functions. Thus, the global increase according to $L_{1}$ and $L_{2}$ was pre-determined approximately using linear regression. The linear regression is performed in order to detect the global tendency of the gain function and not for a modeling purpose. Indeed, the $\mathrm{R}^{2}$ of Gain of time surface models fluctuates around a weak average value of $54.42 \%$ as displayed at fig. 19 .

From fig. 18.a and 18.b, for each hatch distance e, the regression slopes $b_{1}$ and $b_{2}$ of gain of time according to the variables $L_{1}$ and $L_{2}$ are positive, which proves that the global increasing tendency for each time gain surface.

In addition, according to fig. 18.a to 18.c, it is remarkable that the intercepts $b_{0}$ is greater than the slopes $b_{1}$ and $b_{2}$ with a resp. average of 900 times and 700 times. So, we can pronounce a first conclusion that is the intercept $b_{0}$ condition highly the values of the gain of time function. More detailed conclusive remarks about the gain of time are presented in the last section of the paper.

Hence, by reported below the maximal and minimal values of the gain of time:

- A minimal gain of time around $(0.30 \%=|-0.30 \%|)$ is noticed and it corresponds to $e=e_{\text {res }}=25 \mu \mathrm{m}$;

- A maximal gain of time around $(46.29 \%=|-46.29 \%|)$ is noticed and it corresponds to $e=e_{c}=1 \mathrm{~mm}$;

These finding highlights the range of gain of time or gain of energy saved by the new scanning techniques SBP proposed in this paper. Several other discussions could be developed as we fix a set of variables or vary others. 


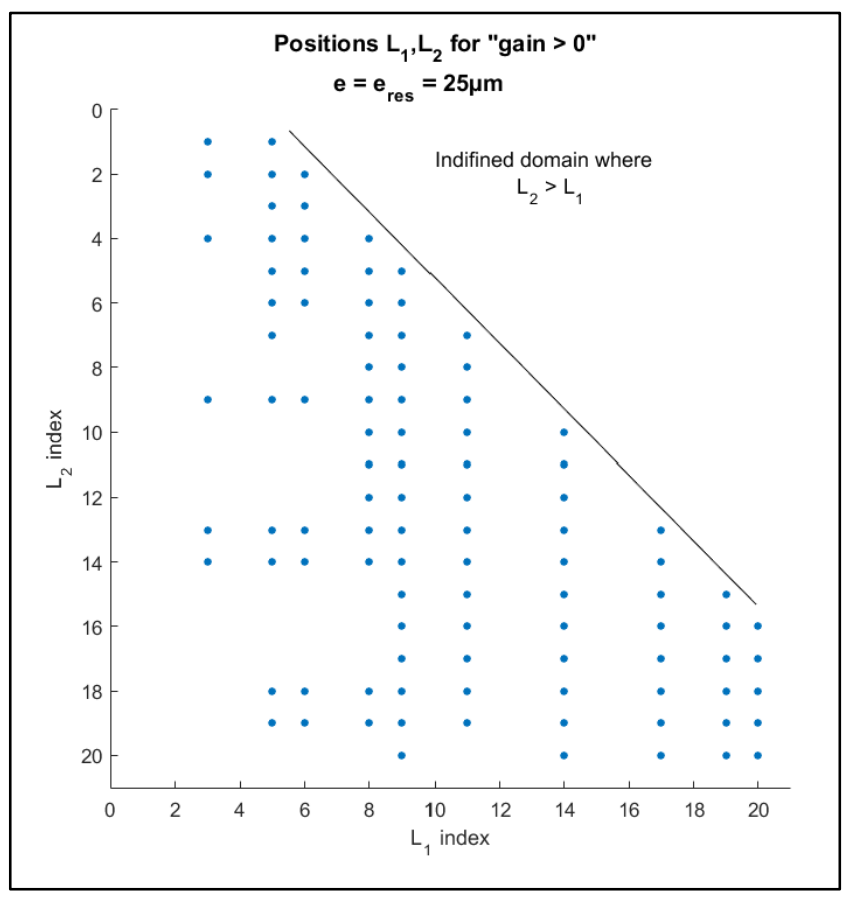

Fig. 17 Positions of positive gain in the case of $e=e_{\text {res }}=25 \mu \mathrm{m}$

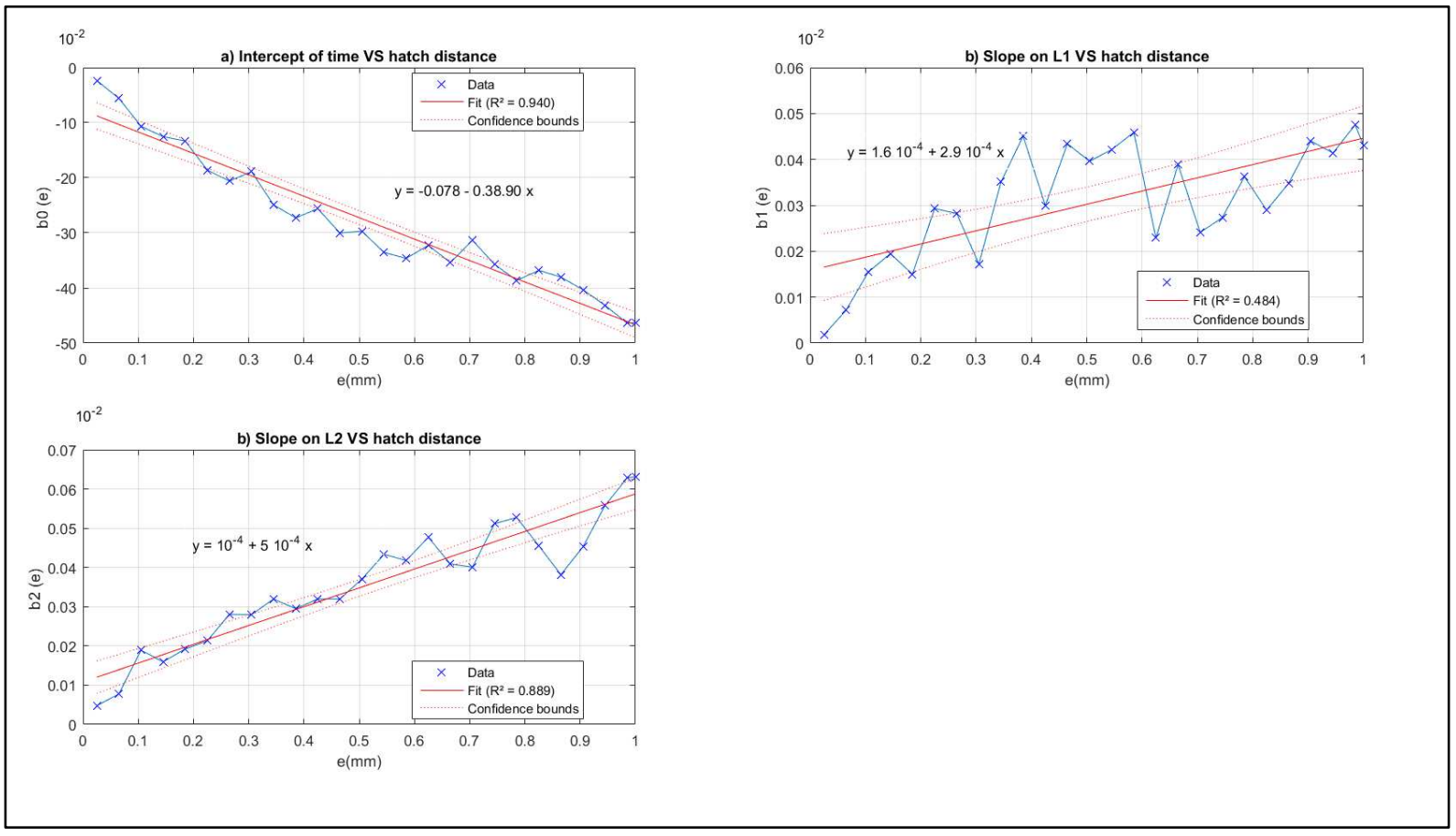

Fig. 18 Tendency parameters of gain of time according to the hatch distance e 


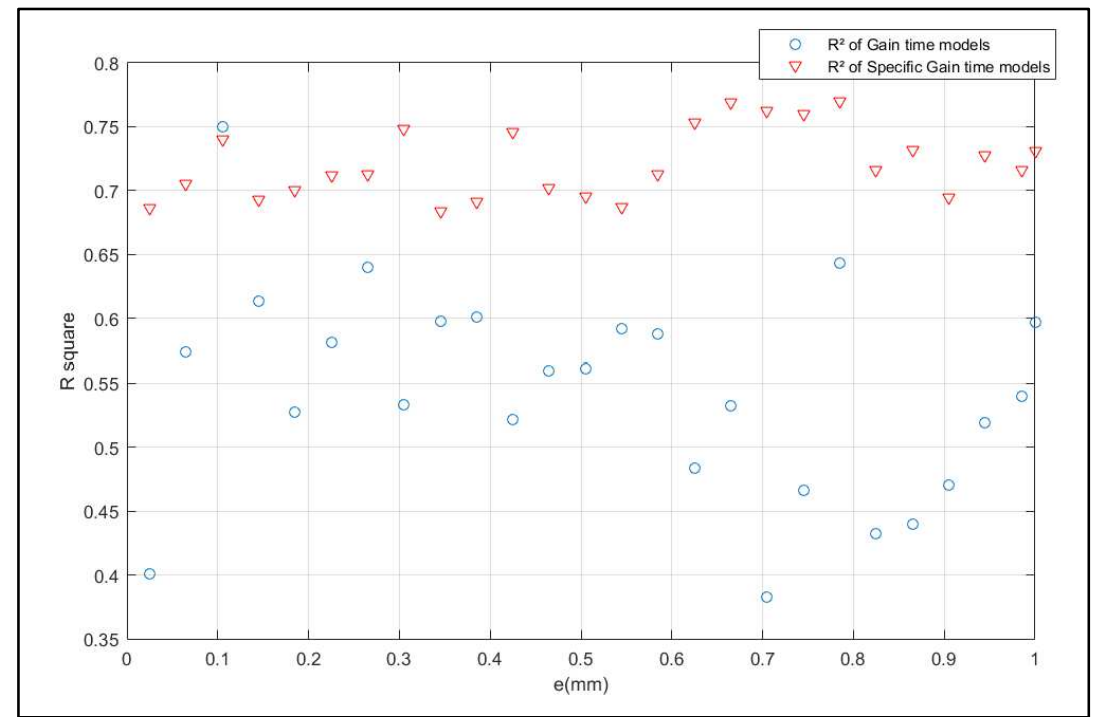

Fig. $19 \mathrm{R}^{2}$ of Gain of Time and $S G^{*}$ surfaces models

\subsubsection{Gain of time per surface unit}

In addition to the above gain of time analysis, the gain of time per surface unit $S G^{*}$ was analyzed graphically. Fig. 20 portrays the subplots of function $S G_{e}^{*}\left(L_{1}, L_{2}\right)$ at different hatch distance values e. $S G^{*}$ surfaces were modeled as linear surfaces as detailed in sect. 2.6. These simplified modeling shows that the linear fitting is interesting since $\mathrm{R}^{2}$ varies around an average value of $74 \%$ as shown by $\mathrm{R}^{2}$ plot of fig. 19 and the sub-titles of fig. 20.

According to the notation of equations (38) and (39) of sect. 2.7, the linear models parameters $\left(b_{0}, b_{1}, b_{2}\right)$ were modeled according to the parameter e (hatch distance). Fig. 21.a-21.c presents the computed values of $\left(b_{0}(e), b_{1}(e), b_{2}(e)\right)$ and the corresponding linear fits; $\mathrm{R}^{2}$ values are higher than $90 \%$ meaning that the fitting is good (refer to the subplot titles).

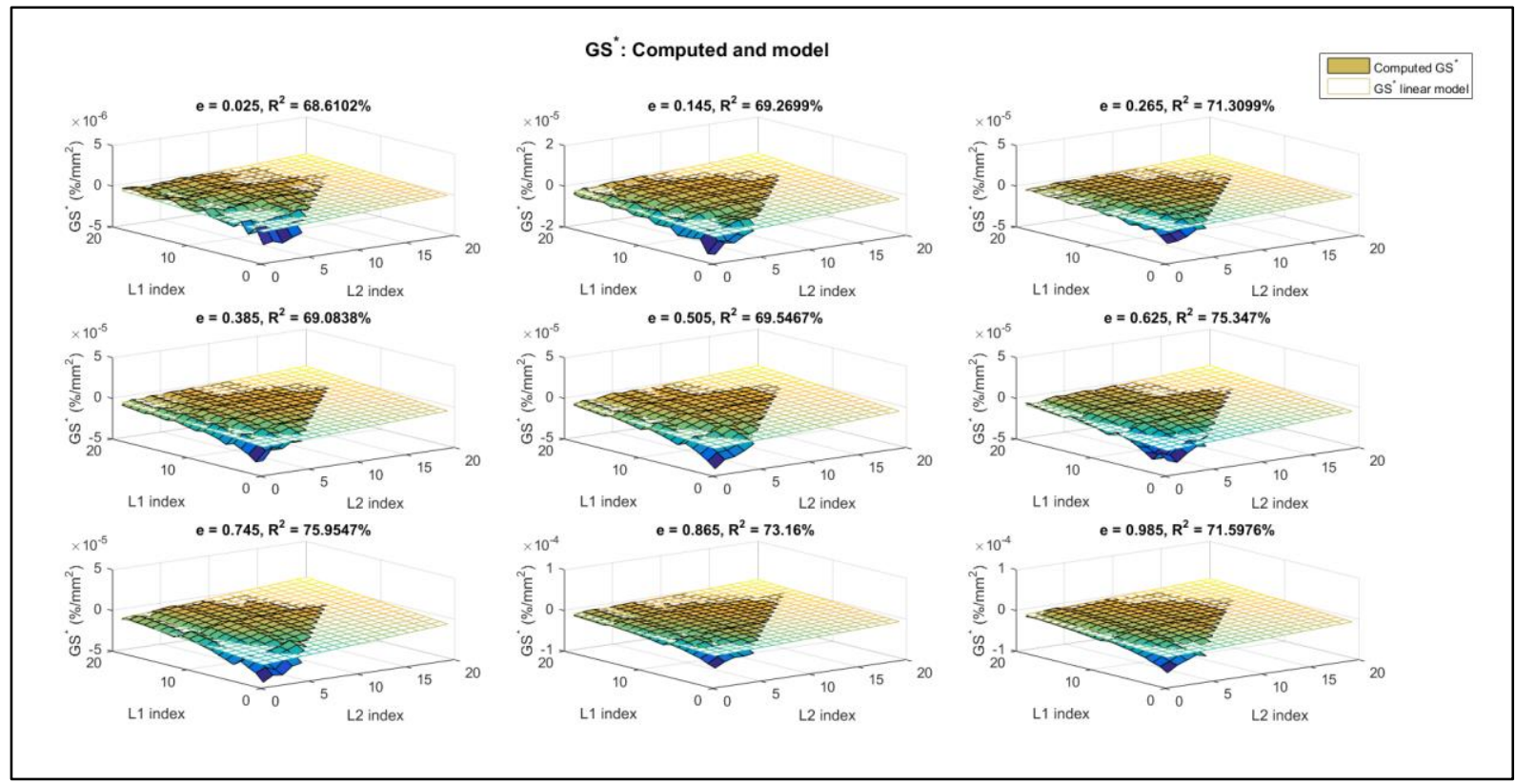

Fig. $20 S G^{*}$ surfaces: Computed and linear models 


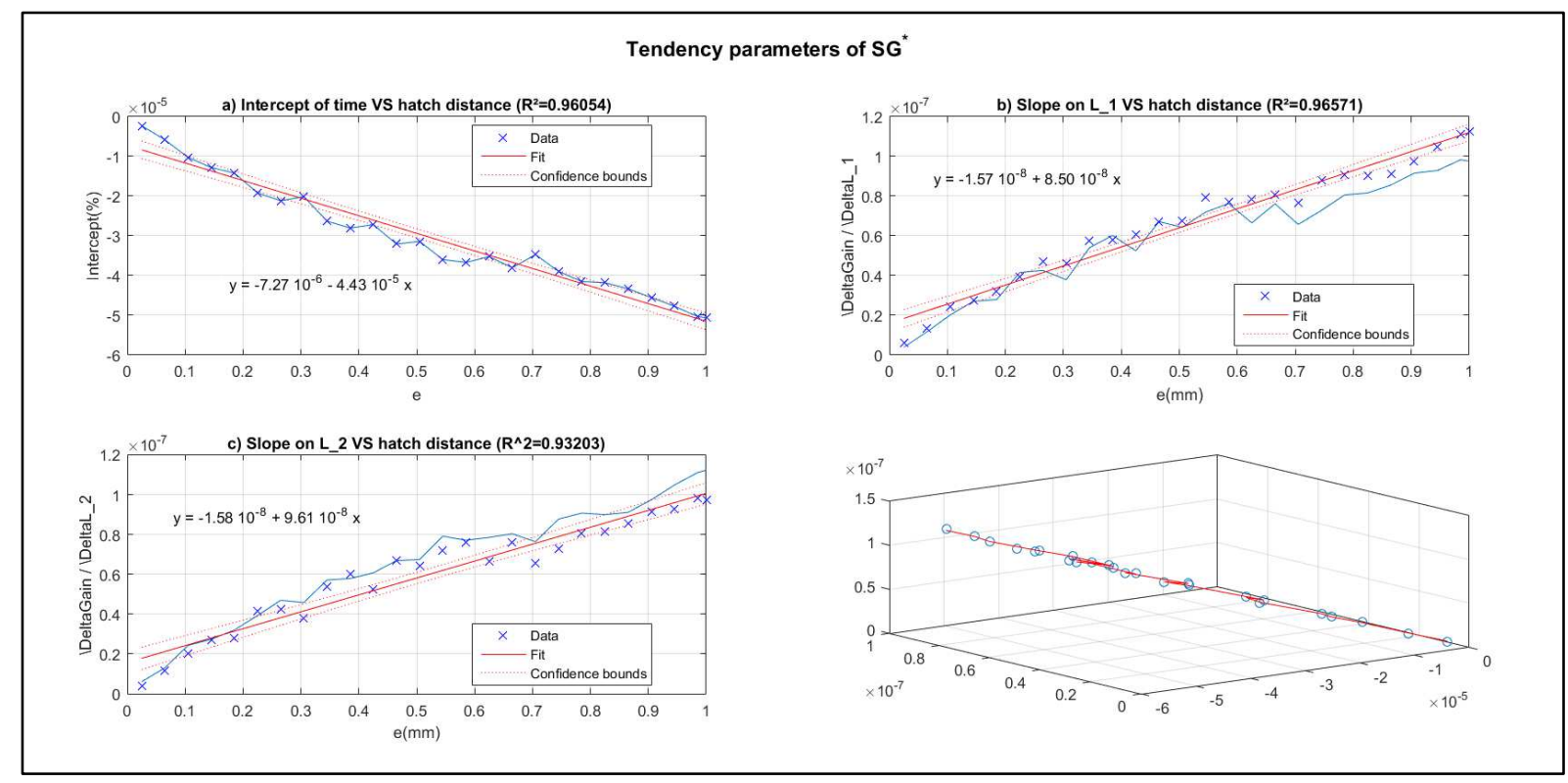

Fig. 21 Plot of the linear models of $S G^{*}$ functions a) plot of $\left(e, b_{0}(e)\right)$ b) plot of $\left(e, b_{1}(e)\right)$ c) plot of $\left(e, b_{2}(e)\right)$

Then, it is possible to model the intercept of the surface and the first order slopes of the function $\widetilde{S G}^{*}$ as a function of the hatch distance $e$, and the geometrical variables $L_{1}$ and $L_{2}$ by a the following models (50):

$$
(50)\left\{\begin{array}{c}
(50.1)\left\{\begin{array}{r}
b_{0}(e)=\widetilde{S G}_{e}^{*}(0,0)=-7.2710^{-6}-4.4310^{-5} e \\
R^{2}=96.05 \%
\end{array}\right. \\
(50.2)\left\{\begin{array}{c}
b_{1}(e)=\frac{\partial \widetilde{S G_{e}^{*}}\left(L_{1}, L_{2}\right)}{\partial L_{1}}=1.5710^{-8}+8.5010^{-8} e \\
R^{2}=96.57 \%
\end{array}\right. \\
(50.3)\left\{\begin{array}{c}
b_{2}(e)=\frac{\partial \widetilde{S G_{e}^{*}}\left(L_{1}, L_{2}\right)}{\partial L_{2}}=1.5810^{-8}+9.6110^{-8} e \\
R^{2}=93.20 \%
\end{array}\right.
\end{array}\right.
$$

Where

$\widetilde{S G_{e}^{*}}:$ is the estimation of $S G_{e}^{*}$ function;

$$
\forall\left(L_{1}, L_{2}\right)_{L_{1}>L_{2}} \in[100,300] \times[50,250]: \widetilde{S G_{e}^{*}}\left(L_{1}, L_{2}\right)=b_{0}(e)+b_{1}(e) L_{1}+b_{2}(e) L_{2}
$$

As it is described in sect. 2.7.

\section{Conclusive remarks and perspectives}

In this paper, a new AM scanning strategy is proposed and it is compared to the classical chess strategy from a productivity perspective. This new technique is based on a scanning perpendicularly to the skeleton of a given flat geometry. As a first application, the new design was dedicated to a rectangular shape to scan. Then, the productivity was assessed by the mean of the computing of total production time for both strategies, and the relative gain of time and energy that are saved by the SBP strategy instead the classical chess. For the cases of study handling and computation, the processing parameters in term of hatch spaces distances and scanning speed were fixed or varied according to the literature review of the SLM processes (table 1 of sect. 1).

Indeed, two cases of study were conducted; the first by fixing a constant rectangle and by computing the time of production and gain of time saved; in the second one, a series of rectangles were included in the simulation and specific gains of time per surface unit were computed and commented. 
For the first case of study, the resolution of the minimization problem $\left(O P_{2}\right)$ began with the assessment of the objective function $T_{S B P}$ and the feasible space that is composed by the inequalities 45.1 to 45.3 . A global increasing tendency of $T_{S B P}$ function was detected according to $n_{C_{x}}$ and $n_{C_{y}}$. First order variations according to these variables were estimated and remarked to be exponentially decreasing with the hatch space distance e (fig. 12). The intercepts $b_{0}(e)$ of $\mathrm{T}_{\mathrm{SBP}}\left(n_{C_{x}}, n_{C_{y}}\right)$ surfaces was detected to have the same behavior. Nevertheless, the modeling precision, especially in term of coefficient of determination $\mathrm{R}^{2}$, was weak since it fluctuates around $54 \%$. Then, the regressive models permitted mainly to observe the first order variation of the objective and constraint function $g_{1}$ of $\left(O P_{2}\right)$. Given these remarks, a global monotonic behavior of $T_{S B P}$ was observed, leading the $\left(O P_{2}\right)$ solution corresponds to $\left(\min \left(n_{C_{x}}\right)=2, \min \left(n_{C_{y}}\right)=2\right)$ for each hatch space distance e. This solution was criticized especially due to the low values of chess pattern decomposition of the $C$ area $(2,2)$ that will absolutely generate coarse scanning patterns. Some remarks related to the residual stress and the non-acceptable deviations of the produced parts are proposed in sect. 4.1.4.

For the second case of study, a new scanning indicator was introduced; the gain of time per surface unit $S G^{*}$ was computed for a series of rectangles $\left(L_{1}, L_{2}\right)$. Unlike the gain of time indicator, $S G_{e}^{*}\left(L_{1}, L_{2}\right)$ surfaces were wellmodeled for each hatch distance e by linear models with a $\mathrm{R}^{2}$ around $74 \%$. In addition, the first order variations of $S G^{*}$ according to $\left(L_{1}, L_{2}\right)$ decrease exponentially according to the hatch space distance e with a coefficient of variation $\mathrm{R}^{2}$ exceeding $90 \%$.

The simulation highlighted that a range of $3 \%$ to $47 \%$ of time and energy gain could be reached by the adoption of the new scanning pattern. In term of gain of time per surface unit, it is possible to save between $4.2310^{-6}\left(\% / \mathrm{mm}^{2}\right)$ and $5.9610^{-3}\left(\% / \mathrm{mm}^{2}\right)$ depending the values of adopted hatch space and the dimensions $\left(L_{1}, L_{2}\right)_{L_{1}>L_{2}}$ of the rectangles. As cited previously, the comparison assessment was discussed at equal hatch space distances in order to minimize the possible major differences of density and matter distribution in the future experiments. But naturally, each scanning pattern and strategy will generate specific mechanical anisotropy of each technique.

Hence, further works will focus on the characterization of the mechanical and metallurgical properties of rectangular parts printed by SBP and chess strategies. Afterward, it would be very interesting to calibrate the productivity optimization problem by taking into consideration the salient results of the experiments into the related constraining system.

\section{Availability of data and material}

No external data were used in this paper.

\section{Competing interests}

The authors declare that they have no competing interests.

\section{Funding}

No funding was attributed to this research.

\section{Authors' contributions}

Pr. El Jai proceeded to the analytical modeling, code implementation and result discussion and interpretation. 
Pr. Akhrif contributed to material science discussion, the choice of processing parameters to adopt, the containing of the optimization problem formulation, and also to the interpretation of the results regarding the material science perspective (residual stress and deformation).

Pr. Saidou initiated the discussion about the proposition of a novel technique of scanning based on the notion of skeleton generation of $2 \mathrm{D}$ shapes; he verified the analytical modeling and contributed to the correction, the calibration of the models, and to the discussion of the results.

\section{References}

[1] https://wohlersassociates.com/press71.html. Accessed 19 Nov. 2020.

[2] Knofius, N, Van der Heijden MC, Zijm WHM (2018). Consolidating spare parts for asset maintenance with additive manufacturing. Int J Prod Econ 208:269-280. https ://doi.org/10.1016/j.ijpe.2018.11.007

[3] RaviPrakash M, Naga SC (2019) Additive manufacturing technology empowered complex electromechanical energy conversion devices and transformers. Appl Mater Today 14:35-50. https ://doi.org/10.1016/j.apmt.2018.11.004

[4] Yung KC, Xiao TY, Choy HS, Wanga WJ, Cai ZX (2018). Laser polishing of additive manufactured CoCr alloy components with complex surface geometry. J Mater Process Technol 262:53-64. https ://doi.org/10.1016/j.jmatp rotec .2018.06.019

[5] El Jai, M. et al. (2020). Mathematical design and preliminary mechanical analysis of the new lattice structure: "GE-SEZ*" structure processed by ABS polymer and FDM technology. Prog Addit Manuf. https://doi.org/10.1007/s40964-020-00148-0

[6] Long, W-J. et al. (2019). Rheology and buildability of sustainable cement-based composites containing microcrystalline cellulose for 3D-printing. J. Cleaner Prod, $239 \quad$ (2019) 118054. https://doi.org/10.1016/j.jclepro.2019.118054

[7] Chen, M. et al. (2020). Rheological parameters and building time of 3D printing sulphoaluminate cement paste modified by retarder and diatomite. Const \& Buil Mater, 234 (2020) 117391. https://doi.org/10.1016/j.conbuildmat.2019.117391

[8] Lowke, D. et al. (2020). Particle bed 3D printing by selective cement activation - Applications material and process technology. Cem \& Concr Res 134, 106077. https://doi.org/10.1016/j.cemconres.2020.106077

[9] Goutham R., Veena, T.R., Babagowda and Srinivasa Prasad, K.R (2017). Study on mechanical properties of recycled Acrylonitrile Butadiene Styrene (ABS) blended with virgin Acrylonitrile Butadiene Styrene (ABS) using Taguchi method. Mater Today: Proceedings 5 (2018) 24836-24845.

[10] Mishurova, T., Artzt, K., Haubrich, J. et al. (2018), New aspects about the search for the most relevant parameters optimizing SLM materials. Addit Manuf. https://doi.org/10.1016/j.addma.2018.11.023

[11] Alafaghani, A. \& Qattawi, A. (2018). Investigating the effect of fused deposition modeling processing parameters using Taguchi design of experiment method. J of Manuf Proc 36, $164-174$. https://doi.org/10.1016/j.jmapro.2018.09.025

[12] Yakout, M., Elbestawi, M.A. and Veldhuis, SC. (2018) A study of thermal expansion coefficients and microstructure during selective laser melting of Invar 36 and stainless steel 316L. Addit Manuf. https://doi.org/10.1016/j.addma.2018.09.035

[13] Z. Zhang, B. Chu, L. Wang, Lu, Z. Comprehensive effects of placement orientation and scanning angle on mechanical properties and behavior of 316L stainless steel based on the selective laser melting process. J Alloys \& Comp (2019). https://doi.org/10.1016/j.jallcom.2019.03.082

[14] Munprom R. \& Limtasiri S. (2017). Optimization of stereolithographic 3D printing parameters using Taguchi method for improvement in mechanical properties. Mater Today: Proceedings 17 (2019) 1768-1773. 
[15] Dong G. et al. (2018). Optimizing process parameters of fused deposition modeling by Taguchi method for the fabrication of lattice structures. Addit Manuf, 19, 62-72. https://doi.org/10.1016/j.addma.2017.11.004

[16] Wankhede, V., Jagetiya, D., Joshi, A. and Chaudhari, R. (2019). Experimental investigation of FDM process parameters using Taguchi analysis. Mater Today Proc., 1-4.

[17] Kumar, S. (2014). Selective Laser Sintering/Melting. Compr Mater Proc, Elsevier, V. 10, 93134. doi:10.1016/b978-0-08-096532-1.01003-7.

[18] Sufiiarov, V.S. et al. (2016). The effect of layer thickness at selective laser melting. 2016 Global Congress on Manufacturing and Management, Proced Eng 174, 126 - 134.

[19] Nguyen, Q.B. et al. (2018). The role of powder layer thickness on the quality of SLM printed parts. Arch Civil Mech Eng, 18, 948 - 955. h ttps://doi.org/10.1016/j.acme.2018.01.015

[20] https://www.sculpteo.com/en/glossary/layer-thickness-definition/. Accessed 19 Nov. 2020.

[21] Järvinen, J-P et al. (2014). Characterization of effect of support structures in laser additive manufacturing of stainless steel. 8th International Conference on Photonic Technologies LANE 2014, Phys Proc 56, 72 - 81.

[22] Jiang, J., Xu, X. and Stringer, J. (2018). Support Structures for Additive Manufacturing: A Review, J. Manuf. Mater. Process. 2, 64. doi:10.3390/jmmp2040064

[23] Deev, A.A., Kuznetcov, P.A. and Petrov, S.N. (2016). Anisotropy of mechanical properties and its correslatin with structure of the stainless steel 316L produced by the SLM method. 9th International Conference on Photonic Technologies - LANE 2016, Phy Proc 83, 789 - 796.

[24] Liverani, E. et al. (2017). Effect of Selective Laser Melting (SLM) process parameters on microstructure and mechanical properties of $316 \mathrm{~L}$ austenitic stainless steel. J of Mater Proc Tech. http://dx.doi.org/10.1016/j.jmatprotec.2017.05.042

[25] Duval-Chaneac, M.S. et al. (2018). Experimental study on finishing of internal laser melting (SLM) surface with abrasive flow machining (AFM). Precis Eng. https://doi.org/10.1016/j.precisioneng.2018.03.006

[26] Mugwagwa, L. et al. (2017). Influence of process parameters on residual stress related distortions in selective laser melting. 15th Global Conference on Sustainable Manufacturing, Proc Manuf, 21, 92-99.

[27] Le Roux, S., Salem, M. and Hor, A. (2018). Improvement of the bridge curvature method to assess residual stresses in selective laser melting. Addit Manuf 22, 320-329. https://doi.org/10.1016/j.addma.2018.05.025

[28] Spierings, A.B., Starr T.L. and Wegener, K., Fatigue performance of additive manufactured metallic parts. Rapid Protyp J, Volume $19 \cdot$ Number $2 \cdot 2013 \cdot 88-94$. DOI : 10.1108/13552541311302932]

[29] Leuders, S. et al. (2014). On the fatigue properties of metals manufactured by selective laser melting - The role of ductility. J. Mater. Res. DOI: 10.1557/jmr.2014.157

[30] Elangeswaran, C. et al. (2019). Effect of post-treatments on the fatigue behavior of 316L stainless steel manufactured by laser powder bed fusion. Int J of Fatig. doi: https://doi.org/10.1016/j.ijfatigue.2019.01.013

[31] Pace, M.L. et al. (2017). 3D additive manufactured 316L components microstructural features and changes induced by working life cycles. Appl Surf Sci. http://dx.doi.org/10.1016/j.apsusc.2017.01.308

[32] Chao, Q. et al. (2017). On the enhanced corrosion resistance of a selective laser melted austenitic stainless steel. Scripta Materialia 141 (2017) 94-98. http://dx.doi.org/10.1016/j.scriptamat.2017.07.037

[33] Olakanmi, E.O., Cochrane R.F. and Dalgarno, K.W. (2015). A review on selective laser sintering/melting (SLS/SLM) of aluminium alloy powders: processing, microstructure, and properties. Prog Mater Sci, 74, 401-477. https://doi.org/10.1016/j.pmatsci.2015.03.002 
[34] Ma, M., Wang, Z. and Zeng, X. (2016). A comparison on metallurgical behaviors of 316L stainless steel by selective laser melting and laser cladding deposition. Mater Sci \& Eng A. http://dx.doi.org/10.1016/j.msea.2016.12.112

[35] Kong, D. et al. (2018). Heat treatment effect on the microstructure and corrosion behavior of 316L stainless steel fabricated by selective laser melting for proton exchange membrane fuel cells. Electrochimica Acta. doi:10.1016/j.electacta.2018.04.188.

[36] Yan, F. et al. (2018). Characterization of nano-scale oxides in austenitic stainless steel processed by powder bed fusion. Scripta Materialia, 155, 104-108. https://doi.org/10.1016/j.scriptamat.2018.06.011

[37] Sun, S-H. et al. (2019). Excellent mechanical and corrosion properties of austenitic stainless steel with a unique crystallographic lamellar microstructure via selective laser melting. Scripta Materialia, 159, 89-93. https://doi.org/10.1016/j.scriptamat.2018.09.017

[38] Gu, D. and Chen, H. (2018). Selective laser melting of high strength and toughness stainless steel parts: The roles of laser hatch style and part placement strategy. Mater Sci \& Eng A. https://doi.org/10.1016/j.msea.2018.04.046

[39] Andreyevna, D.M. et al. (2015). Determination of true temperature in selective laser melting of metal powder using infrared camera. Mater Sci Forum, Vol. 834, 95-104. doi:10.4028/www.scientific.net/MSF.834.95

[40] Li, C., Liu, J. F., Fang, X. Y., \& Guo, Y. B. (2017). Efficient predictive model of part distortion and residual stress in selective laser melting. Addit Manuf, 17, 157-168. doi:10.1016/j.addma.2017.08.014

[41] Strauss, J.T. \& Stucky, M.J. (2016). Laser additive manufacturing processing of a mixture of iron and nickel powders, The 27th Annual Int Solid Freeform Fabric Sympos.

[42] Harrison, N.J., Todd, I. and Mumtaz, K. (2017). Thermal expansion coefficients in Invar processed by selective laser melting. J Mater Sci 52, 10517-10525. https://doi.org/10.1007/s10853-017-1169-4

[43] Yakout M., Elbestawi, M.A. and Veldhuis, S.C. (2018). Density and mechanical properties in selective laser melting of Invar 36 and stainless steel 316L. J Mater Proc Tech. https://doi.org/10.1016/j.jmatprotec.2018.11.006

[44] Kamath, C. et al. (2014). Density of additively manufactured, 316L SS parts using laser powder-bed fusion at powers up to $400 \mathrm{~W}$. The Int J Adv Manuf Tech, 74, 65-78. https://doi.org/10.1007/s00170-014-5954-9

[45] Miranda, G. Et al. (2016). Predictive models for physical and mechanical properties of 316L stainless steel produced by selective laser melting. Mater Sci and Eng A, 657, 43-56. https://doi.org/10.1016/j.msea.2016.01.028

[46] Gong, H. et al. (2015). Influence of defects on mechanical properties of Ti-6Al-4 V components produced by selective laser melting and electron beam melting. Mater and Design, 86, 545-554. http://dx.doi.org/10.1016/j.matdes.2015.07.147

[47] Li, C. et al. (2018). A scalable predictive model and validation for residual stress and distortion in selective laser melting. CIRP Annals, V 67, (1), 249-252. https://doi.org/10.1016/j.cirp.2018.04.105

[48] Robinson, J. et al. (2018). Determination of the effect of scan strategy on residual stress in laser powder bed fusion additive manufacturing. Addit Manuf, 23, 13-24. https://doi.org/10.1016/j.addma.2018.07.001

[49] Felkel, P. and Obderzalek, S. (1998). Straight Skeleton Implementation. Reprinted Proceedings of Spring Conference on Computer Graphics, 210-218, Budmerice, Slovakia.

[50] Rousseau, C. (2001) Mathematiques and Technologie, SUMAT, Springer.

[51] Wu, Q.J. and J. D. Bourland (1999). A Morphology-guided radiosurgery treatment planning and optimization for multiple isocenters, Medic Phy, V. 26, 2151-2160.3. 
[52] Wu, Q.J (2000). Sphere packing using morphological analysis. DIMACS Series in Discr Math and Theor Comp Sci, V. 55, 45-54.

[53] Attali, D. (1995). Squelettes et graphes de Voronoi 2D et 3D. PhD thesis. Joseph Fourier University, Grenoble I, France.

[54] Blum H. (1967). A transformation for extracting new descriptors of shape, Model Percept. Speech Vis. Form 19 (5), 362-380.

[55] Saha, P.K., Borgefors, G., di Baja, G.S. (2017). Chapter 1 - Skeletonization and its applications - A review, Skeletonization. Academic Press, 3-42. https://doi.org/10.1016/B978-0-08-101291-8.00002-X 


\section{Appendix}

\section{A. Study of the area (A)}

\section{a. Bisectors parametrization}

According to the (Spain, 1963), the coordinates of the point $\mathrm{H}$ which is the projection of the point $\mathrm{C}$ on the bisector (B) can be calculated starting from the point $\mathrm{C}$ and the carrier vector $\vec{v}$ of the line (B), as shown on the fig. 7.

Since

$$
\left\{\begin{array}{c}
H \in(B) \\
(B): f(x, y)=a x-y+b=0(51)
\end{array}\right.
$$

Then $\quad a x_{H}-y_{H}+b=0$

\section{b. Expression of the distance $d_{\overrightarrow{C H} / A}$}

For the calculation of the distance $d_{\overrightarrow{C H} / A}=\|\overrightarrow{C H}\|$, the Cartesian distance is utilized according to the coordinates of the point $C\left(x_{C}, y_{C}\right)$ and $H\left(x_{H}, y_{H}\right)$, where:

$$
d_{\overrightarrow{C H} / A}=\|\overrightarrow{C H}\|=\sqrt{\left(x_{H}-x_{C}\right)^{2}+\left(y_{H}-y_{C}\right)^{2}}
$$

Since

$$
\{C\}=(C H) \cap(D)
$$

and

$$
(D): y=L_{2} / 2
$$

thus

$$
y_{C}=L_{2} / 2
$$

The vector $\vec{n}$ that is normal to the bisector (B) is expressed by the following coordinates:

$$
\text { (54) } \vec{n}=\vec{\nabla} f(x, y)=\left\{\begin{array}{l}
n_{x}=\frac{a}{\sqrt{1+a^{2}}} \\
n_{y}=\frac{-1}{\sqrt{1+a^{2}}}
\end{array}\right.
$$

Where $f$ is the implicit function that defines the bisector (B) as described in the equation (51).

To express the $x_{c}$ coordinate, we have to determine the equation of the line $(\mathrm{CH})$.

Since the line $(\mathrm{CH})$ is carried by the vector $\vec{n}$ :

$$
(C H): \frac{-1}{\sqrt{1+a^{2}}} x-\frac{a}{\sqrt{1+a^{2}}} y+C s t=0
$$

The constant "Cst" could be calculated according to the point $\mathrm{H}$ that belong to the line $(\mathrm{CH})$.

Replacing the coordinates of the point $\mathrm{H}$ in the equation (51), we obtain:

$$
\frac{-1}{\sqrt{1+a^{2}}} x_{H}-\frac{a}{\sqrt{1+a^{2}}} y_{H}+C s t=0
$$

Where

$$
\text { (13) } \Leftrightarrow y_{H}=a x_{H}+b
$$

$\Rightarrow \frac{-1}{\sqrt{1+a^{2}}} x_{H}-\frac{a}{\sqrt{1+a^{2}}}\left(a x_{H}+b\right)+C s t=0$

$$
\Rightarrow \text { Cst }=\frac{x_{H}\left(1+a^{2}\right)+a b}{\sqrt{1+a^{2}}}
$$


So the equation of the line $(\mathrm{CH})$ becomes:

$$
(C H): \frac{-1}{\sqrt{1+a^{2}}} x-\frac{a}{\sqrt{1+a^{2}}} y+\frac{x_{H}\left(1+a^{2}\right)+a b}{\sqrt{1+a^{2}}}=0
$$

Since $\forall a \in \mathbb{R}$

$$
\sqrt{\left(1+a^{2}\right)} \neq 0
$$

We find

$$
(C H): x+a y-\left(x_{H}\left(1+a^{2}\right)+a b\right)=0
$$

Since $\quad C \in(C H)$ :

$$
x_{C}+a y_{C}-\left(x_{H}\left(1+a^{2}\right)+a b\right)=0
$$

According to the expression (53):

Finally

$$
\Rightarrow x_{C}+\frac{a L_{2}}{2}-\left(x_{H}\left(1+a^{2}\right)+a b\right)=0
$$

$$
\left\{\begin{array}{l}
x_{C}-x_{H}=a\left(a x_{H}+b-\frac{L_{2}}{2}\right) \\
y_{C}-y_{H}=-\left(a x_{H}+b-\frac{L_{2}}{2}\right)
\end{array}\right.
$$

In addition to the equations (57) and (58), we remark that:

Or

$$
x_{C}-x_{H}=-a\left(y_{C}-y_{H}\right)
$$

$$
y_{C}-y_{H}=-\frac{1}{a}\left(x_{C}-x_{H}\right)
$$

Thus:

$$
\begin{gathered}
d_{\overrightarrow{C H} / A}=\|\overrightarrow{C H}\|=\sqrt{a^{2}\left(y_{H}-y_{C}\right)^{2}+\left(y_{H}-y_{C}\right)^{2}} \\
\Rightarrow d_{\overrightarrow{C H} / A}=\left|y_{H}-y_{C}\right| \sqrt{1+a^{2}}
\end{gathered}
$$

Since

$$
y_{H} \leq y_{C} \Rightarrow\left|y_{H}-y_{C}\right|=y_{C}-y_{H}
$$

Hence

$$
d_{\overrightarrow{C H} / A}=\|\overrightarrow{C H}\|=-a x_{H} \sqrt{1+a^{2}}
$$

\section{c. Expression of the command parameter $x_{H}$}

Let $x_{H}$ be the command scanning space variable. In order to enforce the jumps of the scanning, $x_{H}$ must be considered as a discrete parameter. So $x_{H}$ has to be expressed according to the projection jump step on the $\vec{x}$ axis.

The jump along the bisector (B) is denoted $e_{s}$, its projection, perpendicularly to the skeleton, on the horizontal axis is denoted $e_{s}^{\prime}$ (figure 8).

So, the formulation of $x_{H}$ is given by in the expression (62).

$$
x_{H}=k e_{s}^{\prime}
$$

The geometrical link between $e_{s}$ and $e_{s}^{\prime}$ is described by the figure 22 below. 


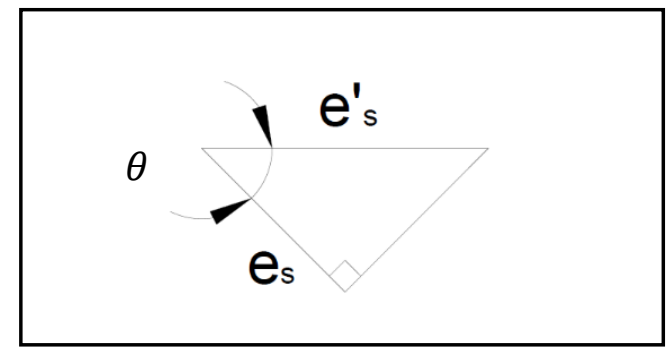

Fig 22 Relation between $e_{s}$ and $e_{S}^{\prime}$

Thus

It implies that

$$
\text { (63) }\left\{\begin{array}{c}
\text { (fig. 9) } \Rightarrow \cos (\theta)=e_{s} / e_{s}^{\prime} \\
(\text { fig. } 6) \Rightarrow\left\{\begin{array}{c}
\cos (\theta)={ }^{\alpha_{1} L_{1} / \Lambda} \\
\Lambda=\sqrt{\left(\alpha_{1} L_{1}\right)^{2}+\left(\frac{L_{2}}{2}\right)^{2}}
\end{array}\right.
\end{array}\right.
$$

Finally

$$
e_{s}^{\prime}=\frac{e_{s} \Lambda}{\alpha_{1} L_{1}}=\frac{e_{s}}{\alpha_{1} L_{1}} \sqrt{\left(\alpha_{1} L_{1}\right)^{2}+\left(\frac{L_{2}}{2}\right)^{2}}
$$

$$
e_{s}^{\prime}=e_{s} \sqrt{1+\left(\frac{L_{2}}{2 \alpha_{1} L_{1}}\right)^{2}}
$$

Let's denote

$$
\beta=\sqrt{1+\left(\frac{L_{2}}{2 \alpha_{1} L_{1}}\right)^{2}}
$$

And

$$
e_{s}^{\prime}=\beta e_{s} \quad(66)
$$

In the case of this study, the angle $\theta$ is equal to $\frac{\pi}{4}$, so the link between the jumps $e_{s}$ and $e_{s}^{\prime}$ could be directly be expressed by the expression (67).

$$
\cos (\theta)=\frac{\sqrt{2}}{2}=e_{s} / e_{s}^{\prime}(67)
$$

For more genericity of the modeling approach, the author wanted to express the final expression of the total scanning length according to all geometrical parameters as dummy variables. Thereafter in the simulation, the numerical values will appropriately replace the problem parameters.

So, according to (62), the expression of the command $x_{H}$ becomes $x_{H_{A}}$ such that:

$$
\text { (68) }\left\{\begin{array}{c}
x_{H}(k)=x_{H} / A \\
x_{H_{A}}(k)=k e_{s}^{\prime}=k \beta e_{S} \\
1 \leq k \leq k_{A}
\end{array}\right.
$$

Where $x_{H} / A$ is the restriction of $x_{H}$ on the area $\mathrm{A}$, function of the index $k$.

The index $k$ is the increment of the command, it begins by $k_{1}=1$ till a limit value $k_{\mathrm{A}}$ that limits the area (A) as depicted in fig. 10. 
From fig. 10, the $k$ index takes $k_{\mathrm{A}}$ value when the point $\mathrm{C}$ reaches the right limits of the area (A). This condition is expressed by:

$$
\left\{\begin{array}{l}
x_{C}\left(k_{A}\right)=\alpha_{1} L_{1} \\
x_{H}\left(k_{A}\right)=k_{A} \beta e_{S}
\end{array}\right.
$$

Replacing (69) and (70) in the equation (57):

$$
\alpha_{1} L_{1}=\left(a^{2}+1\right) k_{A} \beta e_{s}+a\left(b-\frac{L_{2}}{2}\right)
$$

Thus, $k_{A}$ is computed using the expression (71).

$$
k_{A}=\frac{\alpha_{1} L_{1}}{\left(a^{2}+1\right) e_{s} \beta}
$$

\section{d. Correction of the index $k_{A}$}

Since $k_{A}$ is an integer number, the corrected $k_{A_{C}}$ must is expressed by:

$$
k_{A_{C}}=E\left(\frac{\alpha_{1} L_{1}}{\left(a^{2}+1\right) e_{s} \beta}\right)
$$

and

$$
x_{H_{A}}=\frac{\alpha_{1} L_{1}}{1+a^{2}}
$$

\section{e. Productive length of the area (A)}

On the area (A), the productive length $L_{P_{A}}$ is corresponds to the following summation:

$$
L_{P_{A}}=\sum_{k=1}^{k_{A_{C}}} d_{\overrightarrow{C H} / A}+\left(d_{\overrightarrow{C H} / A}\right)_{f}
$$

Where $\left(d_{\overrightarrow{C H} / A}\right)_{f}$ is the distance $C H$ at the limit of the area (A). According to the expression (61):

$$
\left(d_{\overrightarrow{C H} / A}\right)_{f}=-a x_{H_{A}} \sqrt{1+a^{2}}
$$

Where $x_{H_{A}}$ is expressed by the equation (73).

So

$$
L_{P_{A}}=\sum_{k=1}^{k_{A}} d_{\overrightarrow{C H} / A}+\left(d_{\overrightarrow{C H} / A}\right)_{f}=\sqrt{1+a^{2}} \sum_{k=1}^{k_{A_{C}}}\left(-a x_{H}\right)+\left(-a x_{H_{A}}\right) \sqrt{1+a^{2}}
$$

Since $x_{H}=k e_{s}^{\prime}(62)$

$$
\begin{array}{r}
L_{P_{A}}=\sqrt{1+a^{2}}\left[-\frac{a}{2} k_{A_{C}}\left(k_{A_{C}}+1\right) e_{S}^{\prime}+\left(-a x_{H_{A}}\right)\right] \\
L_{P_{A}}=\sqrt{1+a^{2}}\left[-\frac{a \beta}{2} e_{S} k_{A_{C}}\left(k_{A_{C}}+1\right)+\left(-a x_{H_{A}}\right)\right] \\
L_{P_{A}}=-a \sqrt{1+a^{2}}\left[\frac{\beta}{2} e_{S} k_{A_{C}}\left(k_{A_{C}}+1\right)+x_{H_{A}}\right]
\end{array}
$$




\section{f. Non-productive length of the area (A)}

On the other hand, the non-productive length $L_{N P_{A}}$ is calculated by multiplying the jumps lengths $e_{s}$ and $e_{S}^{\prime}$ by their respective number of repetitions.

The author analyzes two cases:

- $\quad k_{A_{C}}$ is an impair number:

$$
\begin{gathered}
L_{N P_{A}}=\sum_{k=1}^{E\left({ }^{k}{ }_{A} / 2\right)}\left(e_{s}+e_{s}^{\prime}\right)=\sum_{k=1}^{E\left({ }^{k}{ }_{A} / 2\right)} e_{s}(1+\beta) \\
L_{N P_{A}}=E\left({ }^{k_{A_{C}} / 2}\right)(1+\beta) e_{S}
\end{gathered}
$$

- $\quad k_{A_{C}}$ is pair number:

$$
\begin{array}{r}
L_{N P_{A}}=\sum_{k=1}^{\frac{k_{A_{C}}}{2}-1} e_{s}+\sum_{k=1}^{\frac{k_{A_{C}}}{2}} e_{s}^{\prime} \\
L_{N P_{A}}=\left(\frac{k_{A_{C}}}{2}-1\right) e_{s}+\frac{k_{A_{C}}}{2} e_{s}^{\prime}
\end{array}
$$

\section{B. Study of the area (B)}

Considering the rectangular geometry that is analyzed in this paper, the region (A) and (B) are symmetrical according to the red line of fig. 10. Then the productive and non-productive lengths formulated for the area (A) are adopted for the area (B).

\section{Study of the area $\mathrm{C}$}

According to fig. 7, the area (C) corresponds to a simple rectangle. The scanning schema to adopt can be composed by the classical chess policy or it is possible to create a second level of skeleton scanning on this area. In order to proceed as simple as possible for this first step of skeleton scanning modeling, the authors decided to adopt the classical chess strategy on the area (C). This is adequate with the scanning principal adopted in this study, since the chess schema remains perpendicular and parallel to the skeleton. Thus, the constraint of the perpendicularity to the skeleton is quite ensured.

The productive length $L_{P_{C}}$ and non-productive length $L_{N P_{C}}$ can be formulated similarly from equations (1-5) that were developed for the chess scanning strategy in sect. 1. As expressed in the equations (1-5), the scanning schema can be fixed according to the number or the dimensions of the patterns of fig. 5 and 6 .

After hand development adaptation, the productive and the non-productive lengths of the area (C) are expressed respectively by the equations (77) and (78).

$$
L_{P_{C}}=\frac{N_{1} \alpha_{1} L_{1}}{2}\left(\frac{1}{n_{C_{x}}}\left(\frac{L_{2}}{2 n_{C_{y}} e_{s}}+1\right)+\frac{1}{n_{C_{y}}}\left(\frac{\alpha_{2} L_{1}}{2 n_{C_{x}} e_{s}}+1\right)\right)
$$

Since

$$
\left\{\begin{array}{c}
\alpha_{1} L_{1}=\frac{L_{2}}{2} \\
\alpha_{2} L_{1}=L_{1}-L_{2}
\end{array}\right.
$$

the productive and non-productive lengths of $\mathrm{C}$ area become: 


$$
\begin{gathered}
L_{P_{C}}=\frac{N_{1 C} L_{2}}{4}\left(\frac{1}{n_{C_{x}}}\left(\frac{L_{2}}{2 n_{C_{x}} n_{C_{y}} e_{S}}+1\right)+\frac{1}{n_{C_{y}}}\left(\frac{\left(L_{1}-L_{2}\right)}{2 n_{C_{x}} e_{s}}+1\right)\right) \text { (77) } \\
L_{N P_{C}}=\frac{L_{2}}{2}\left(\frac{N_{1 C}}{n_{C_{y}}}+\frac{N_{2 C} L_{2}}{2 n_{C_{x}}}\right)(78) \\
\text { (79) }\left\{\begin{array}{c}
\text { if }\left(\left(n_{C_{x}} \text { is pair }\right) \text { or }\left(n_{C_{y}} \text { is pair }\right)\right): N_{1 C}=N_{2 C}=\frac{n_{C_{x}} n_{C_{y}}}{2} \\
\text { else }\left\{\begin{array}{c}
\text { if chess begins with pattern } 1: N_{1 C}=E\left(\frac{n_{C_{x}} n_{C_{y}}}{2}\right)+1 \\
\text { if chess begins with pattern } 2: N_{2 C}=E\left(\frac{n_{C_{x}} n_{C_{y}}}{2}\right)+1 \\
N_{1}+N_{2}=n_{C_{x}} n_{C_{y}}
\end{array}\right.
\end{array}\right.
\end{gathered}
$$

\section{Where}

$N_{I C}$ : is the total number of chess pattern 1 (fig. 5);

$N_{2 C}$ : is the total number of pattern 2 (fig. 5);

$n_{C_{x}}:$ is the number of divisions of the dimension of the rectangle according to the pattern along the axis $\vec{x}$;

$n_{C_{y}}$ : is the number of divisions of the dimension of the rectangle according to the pattern along the axis $\vec{y}$; 


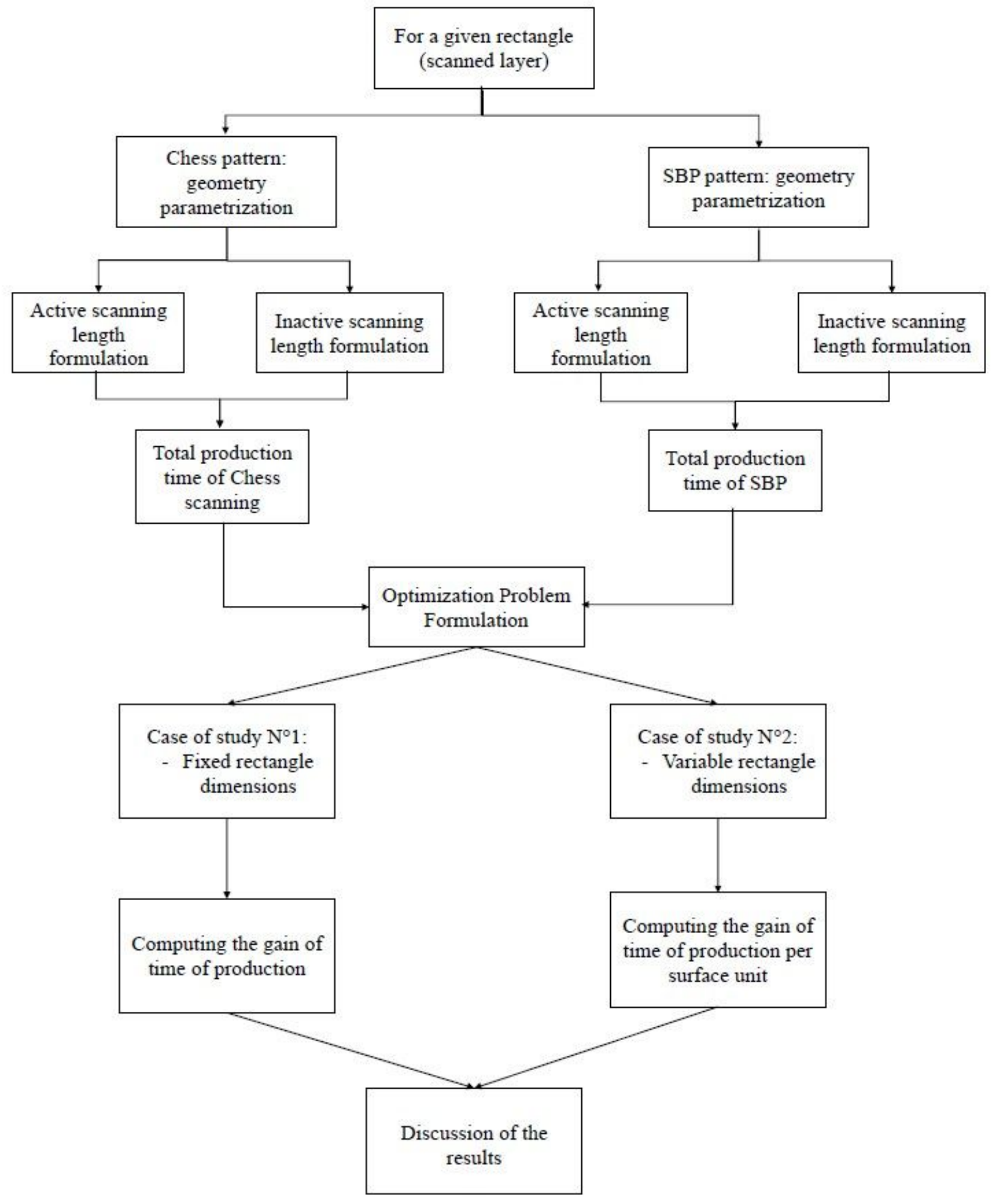

Figure 1

Workflow of the study 


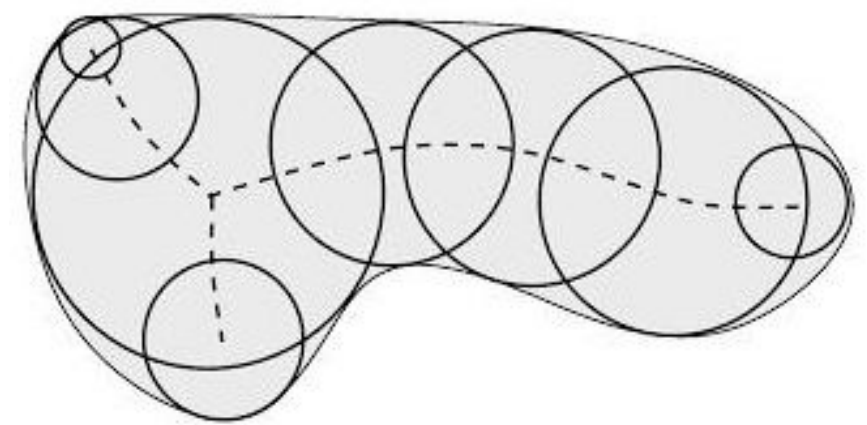

Figure 2

Internal disks and maximal disks (balls) of a 2-D shape, Building the shape skeleton [53]

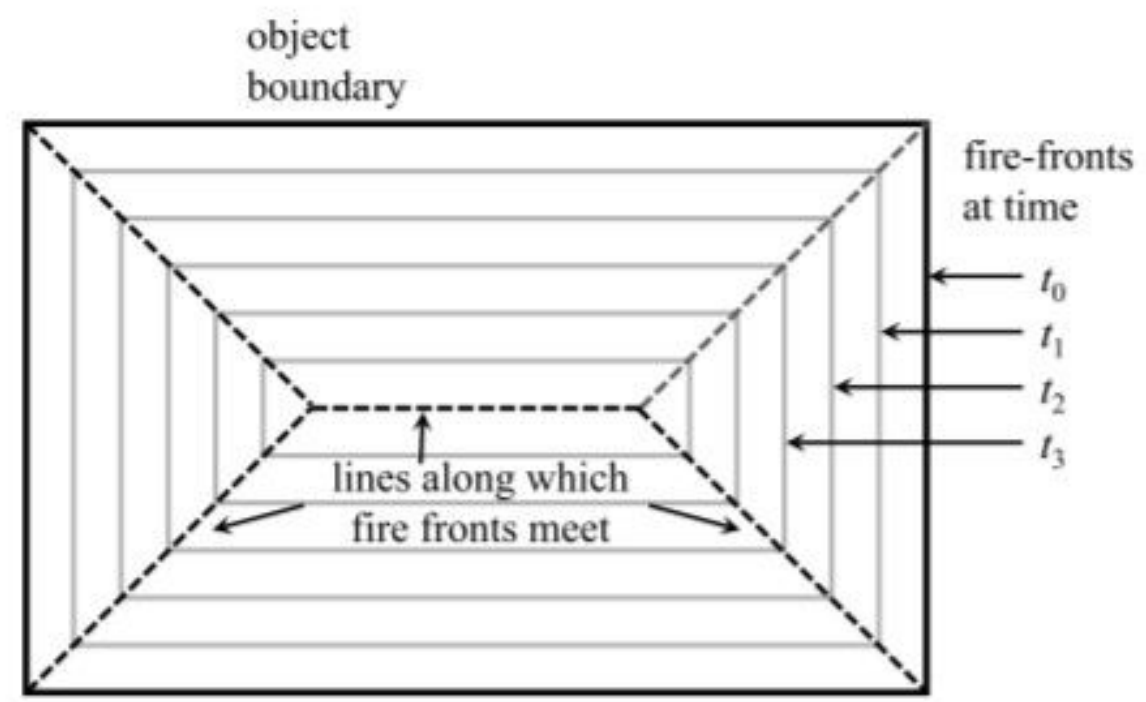

Figure 3

Skeleton of a rectangle depicted by a set of lines as the lieu in which the fire fronts meet [55] 


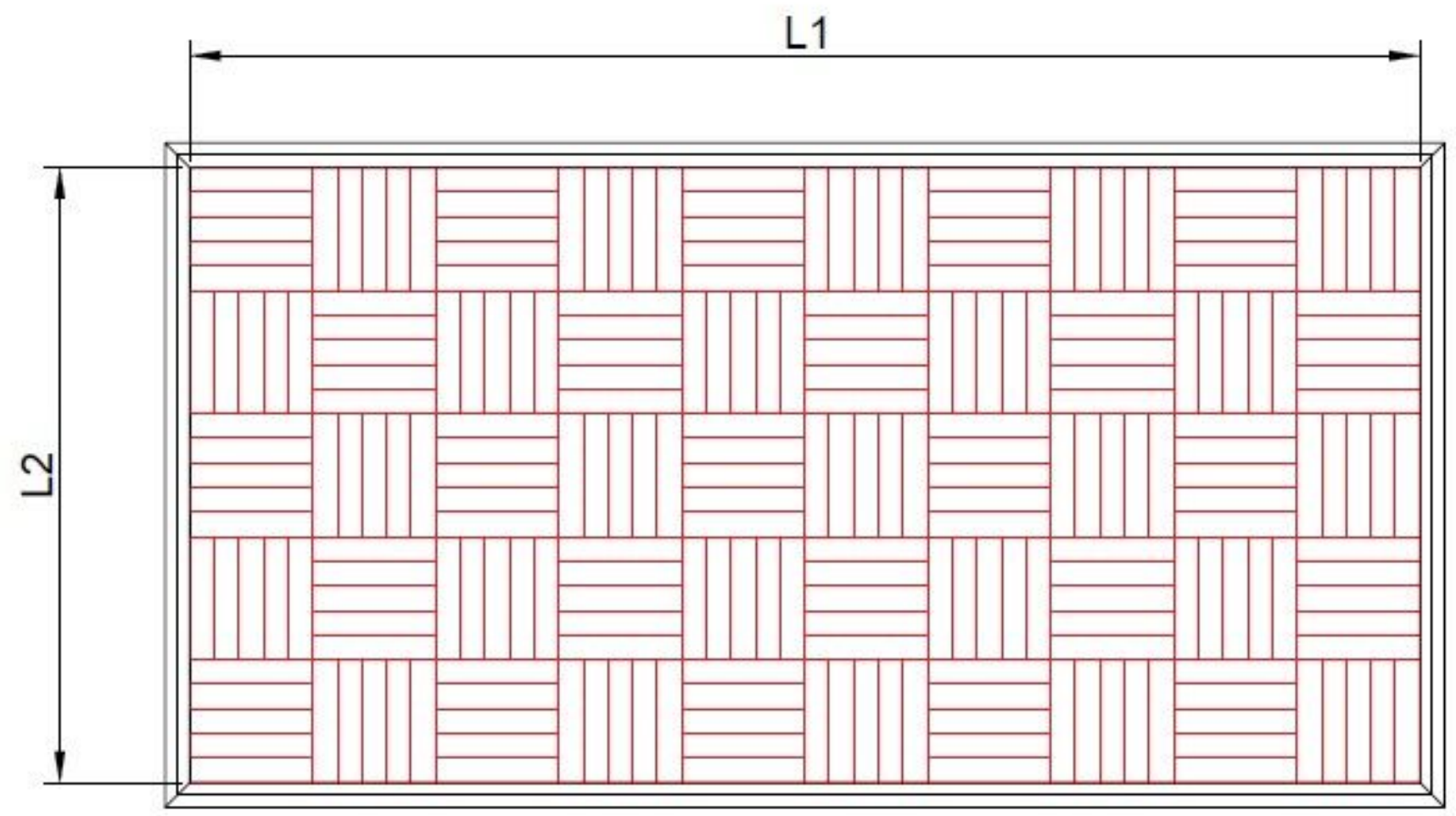

Figure 4

Classical Chess scanning policy in the Additive Manufacturing processes

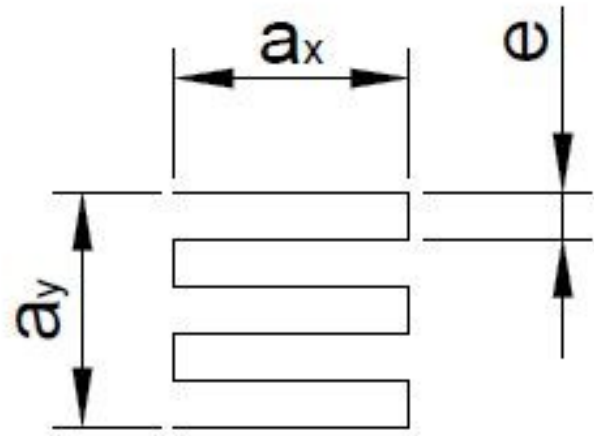

Figure 5

Chess scanning pattern $n^{\circ} 1$ 


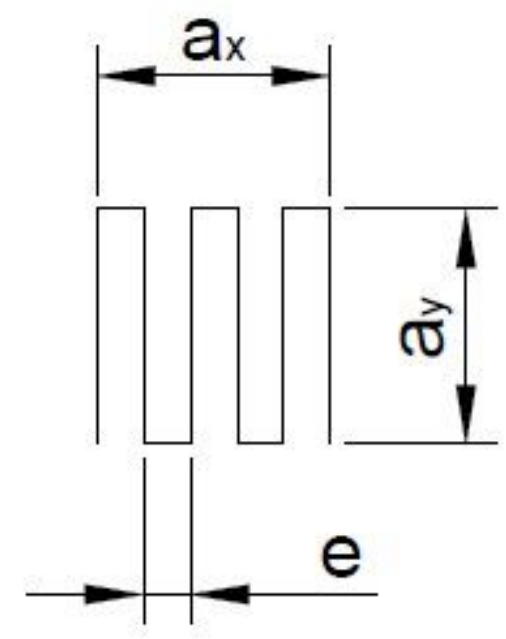

Figure 6

Chess scanning pattern $n^{\circ} 2$ 


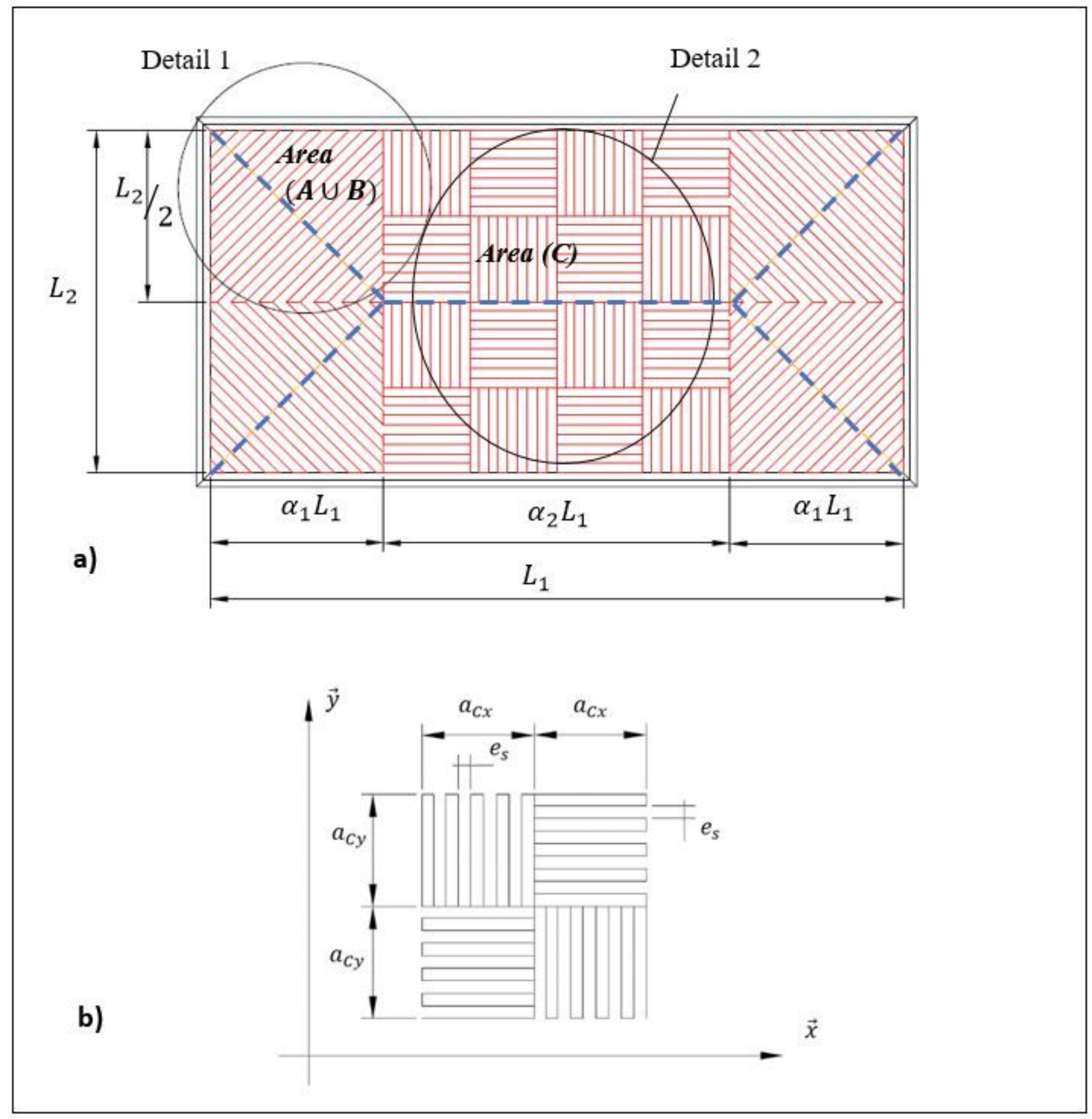

Figure 7

Skeleton Based Perpendicularly strategy scan pattern a) Global pattern b) $\mathrm{C}$ area pattern and the corresponding geometrical parameterization 


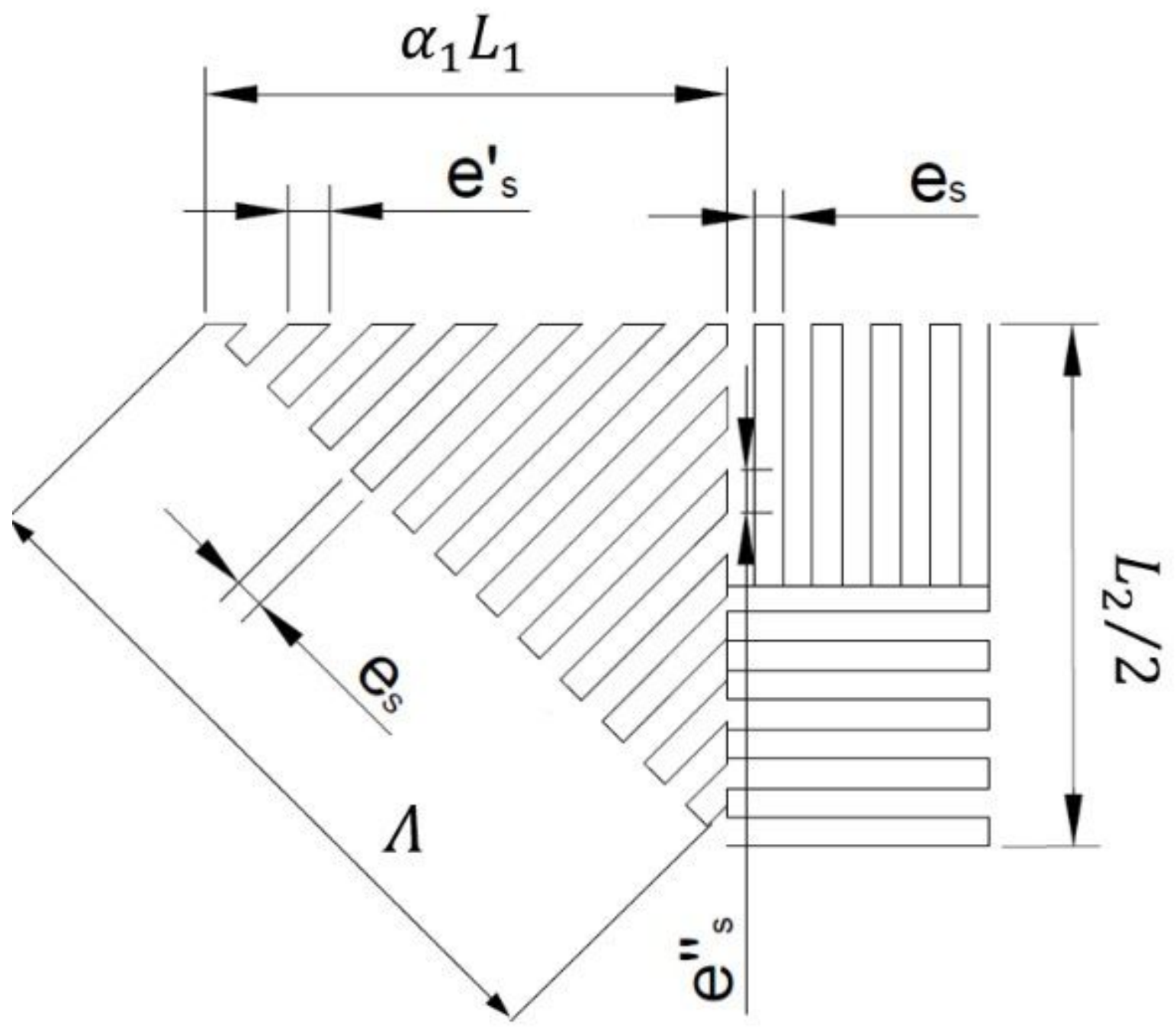

Figure 8

Detail 1 of fig. 7: Geometric parametrization of the (SBP) scanning strategy 


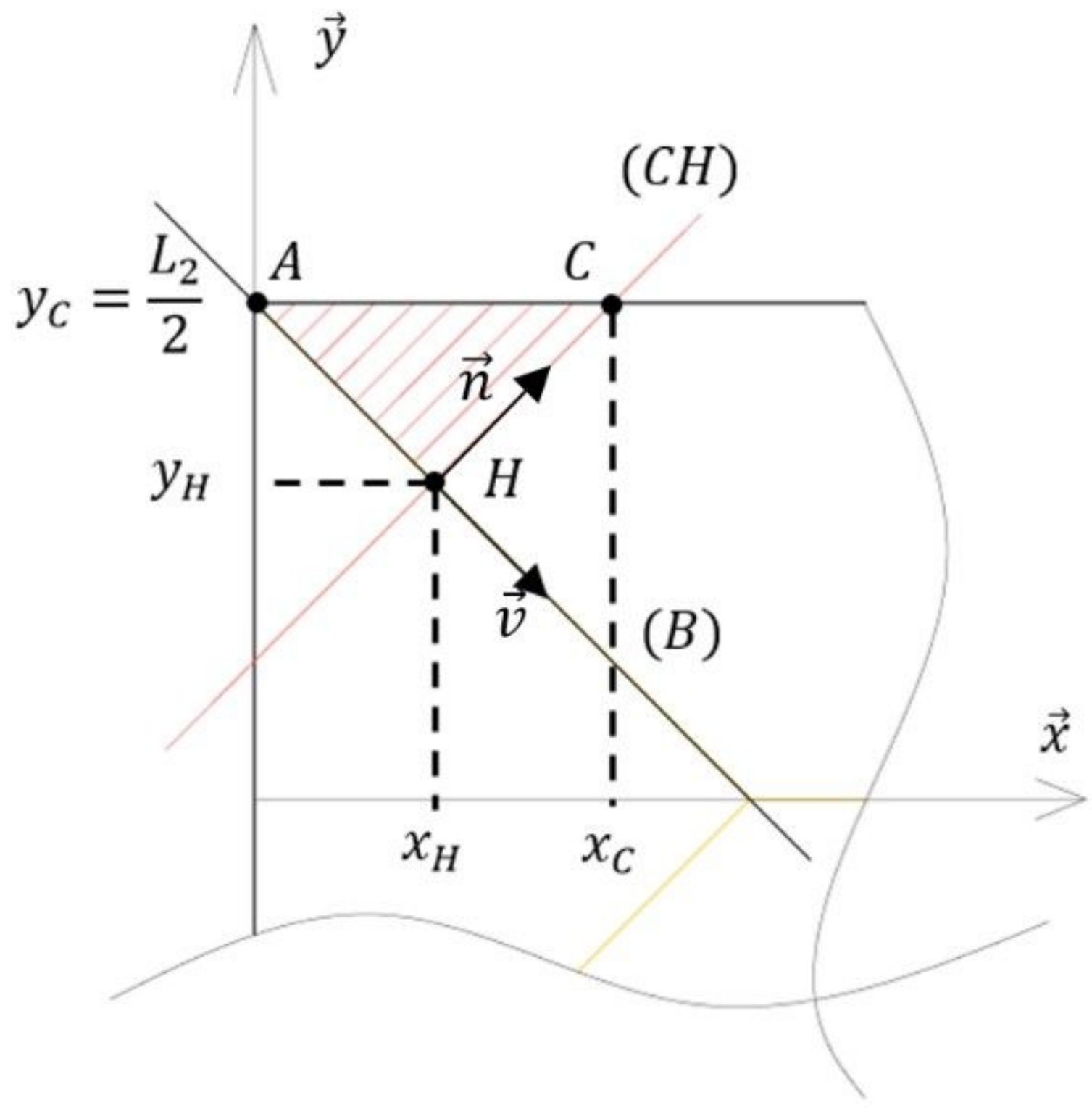

Figure 9

Positions and coordinates of the characteristic points $\{A, C, H\}$ 


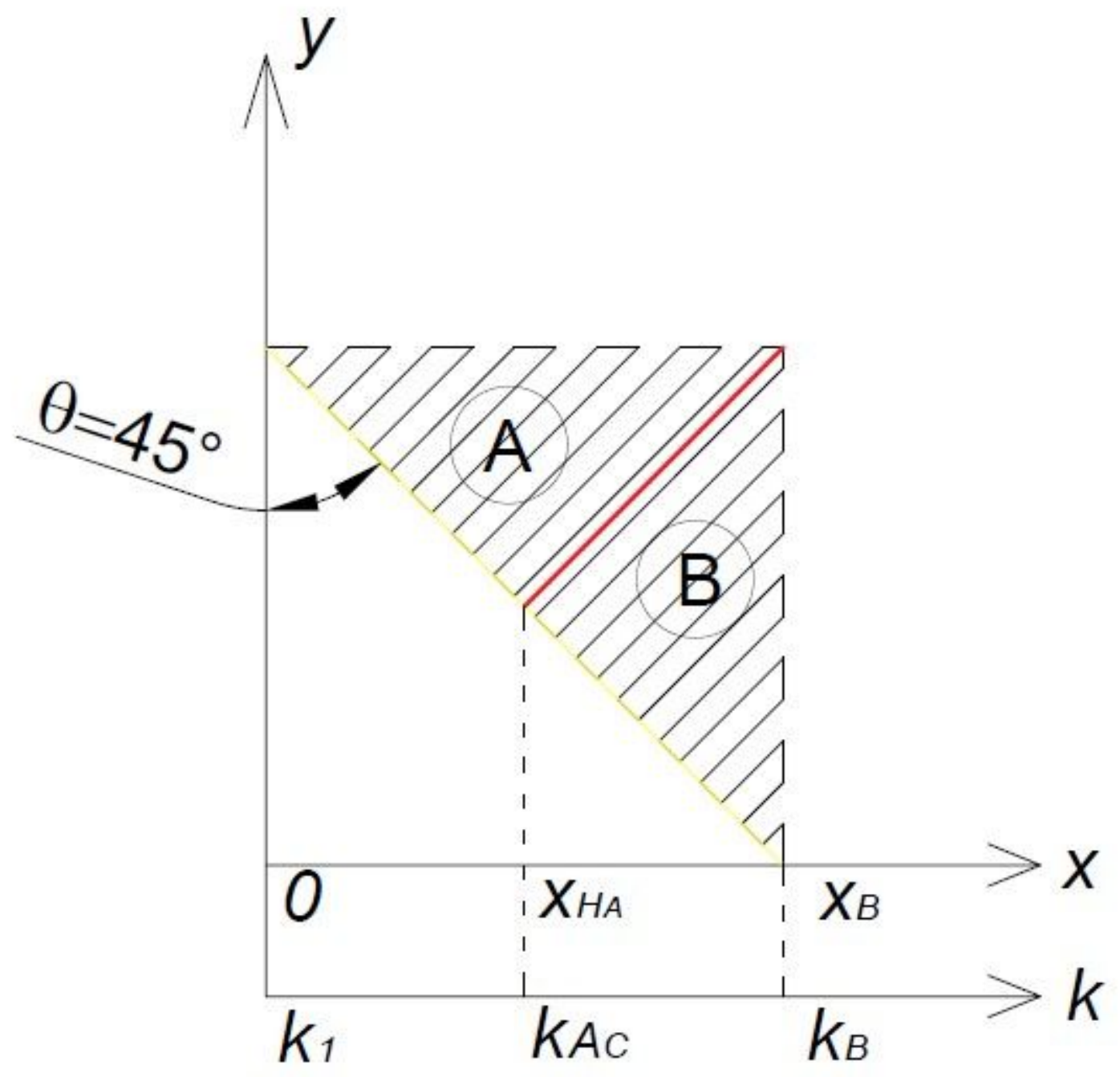

Figure 10

Decomposition of the first quadrant related to the left upper corner of the rectangle 


\section{"SBP strategy prod. time" VS "( $\left.\mathrm{n}_{\mathrm{Cx}_{x}}, \mathrm{n}_{\mathrm{C}_{y}}, \mathrm{e}\right) "$}
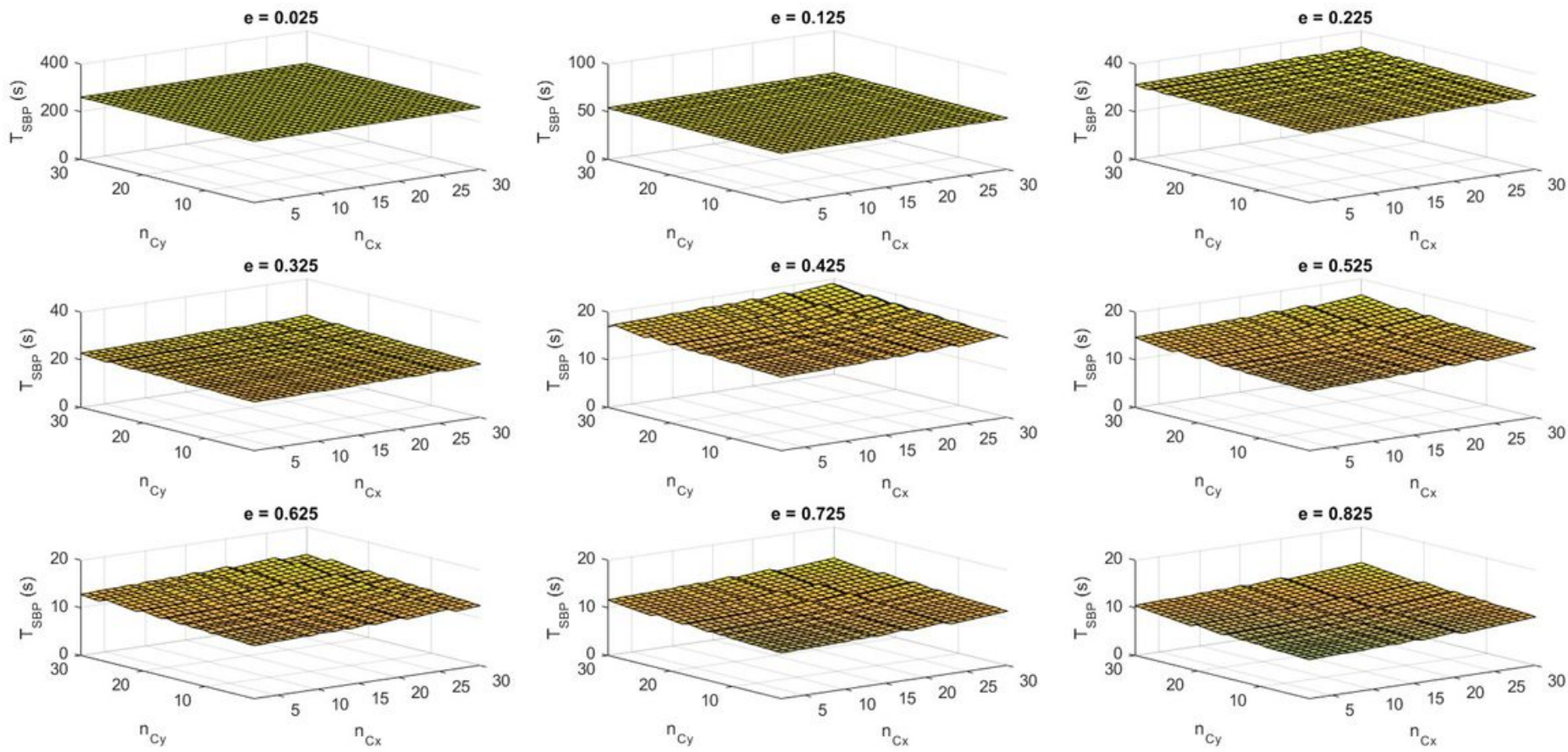

\section{Figure 11}

Production time of the SBP strategy according to (n_Cx, n_Cy, e)

a)

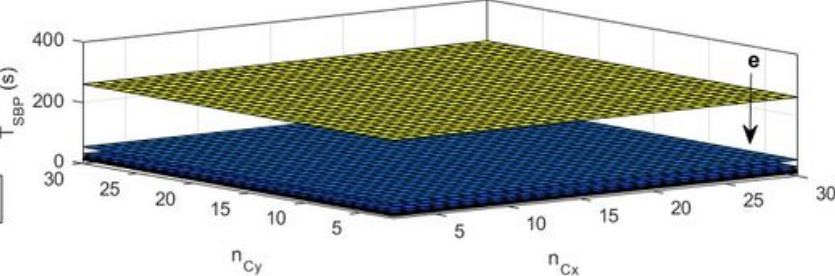

c)

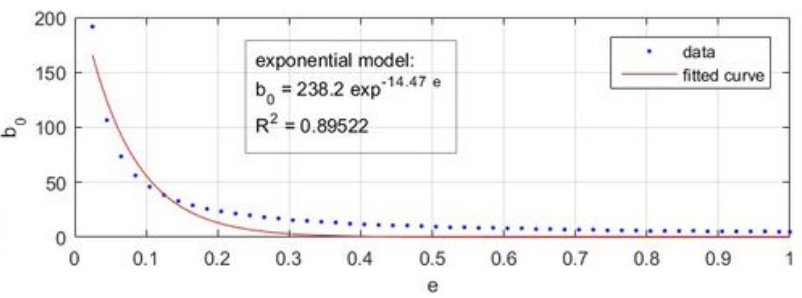

e)

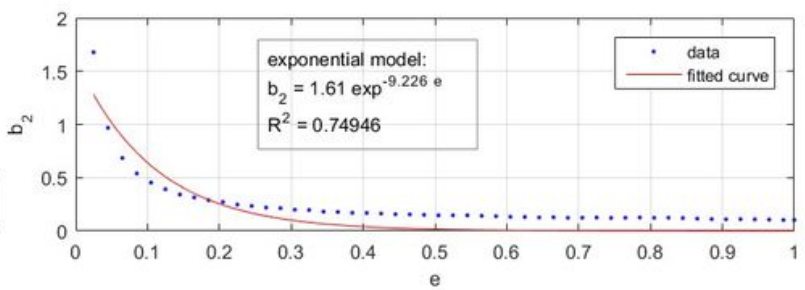

b)

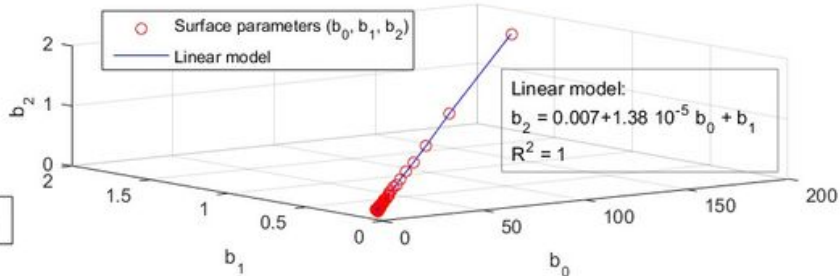

d)

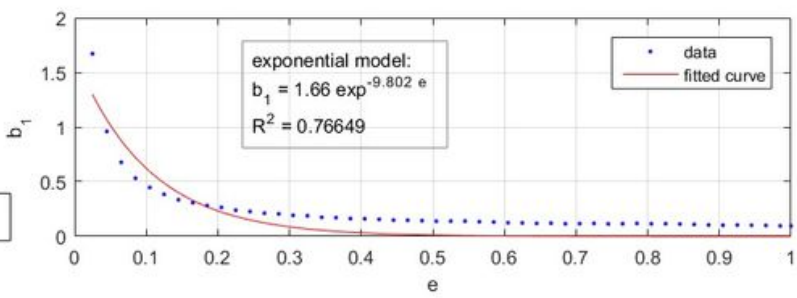

Figure 12 
Variation of the T_SBP surfaces parameters a) Superposition of the surfaces family T_SBP b) 3D-plot of (b_0,b_1,b_2 ) c) Evolution of the intercepts b_0 according to hatch space e d) Evolution of first order variation b_1 according to hatch space e e) Evolution of first order variation b_2 according to hatch space e

\section{Feasible space according to $\mathrm{g}_{1}$ constraint (45.1)}
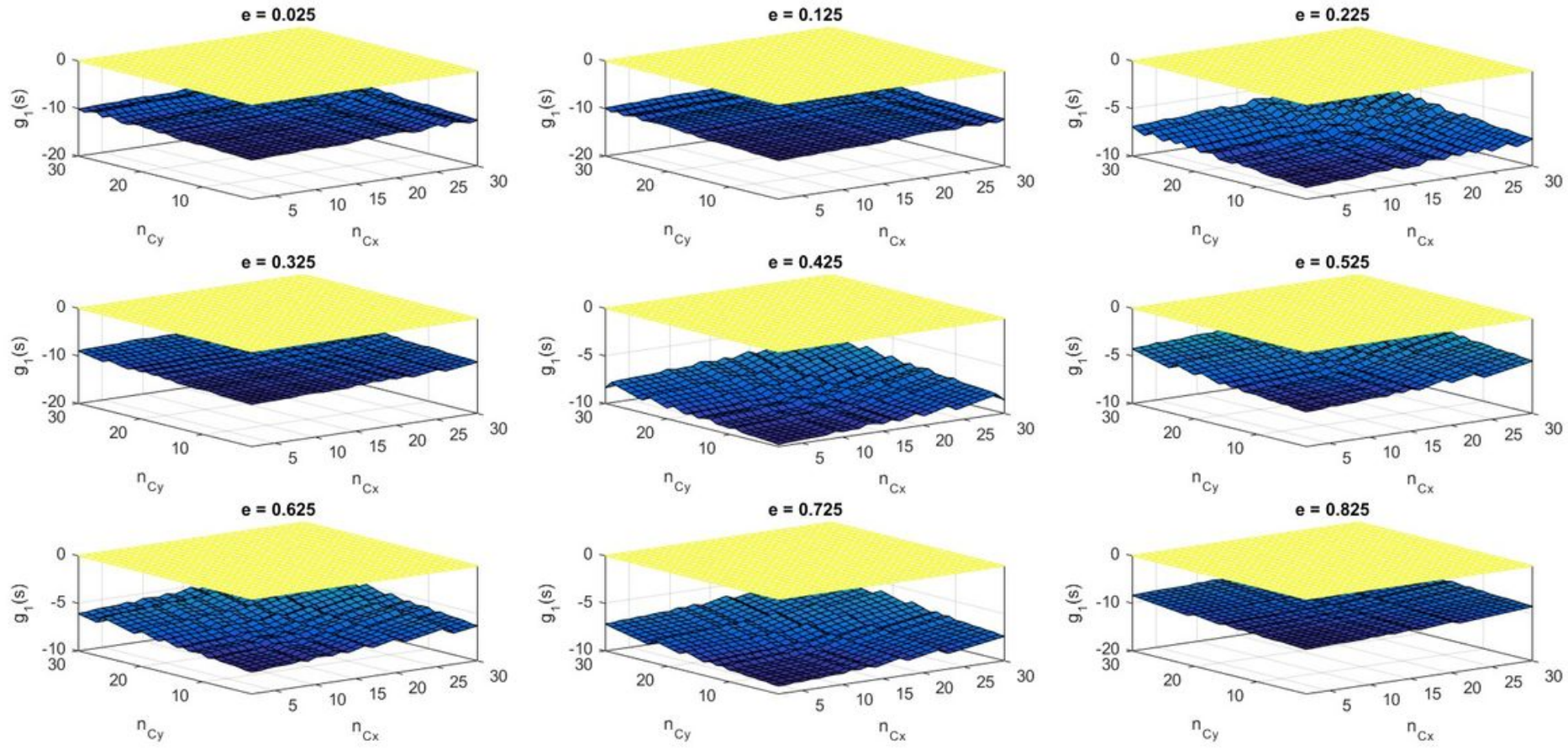

Figure 13

g_1 function values according to (n_Cx, n_Cy, e) 


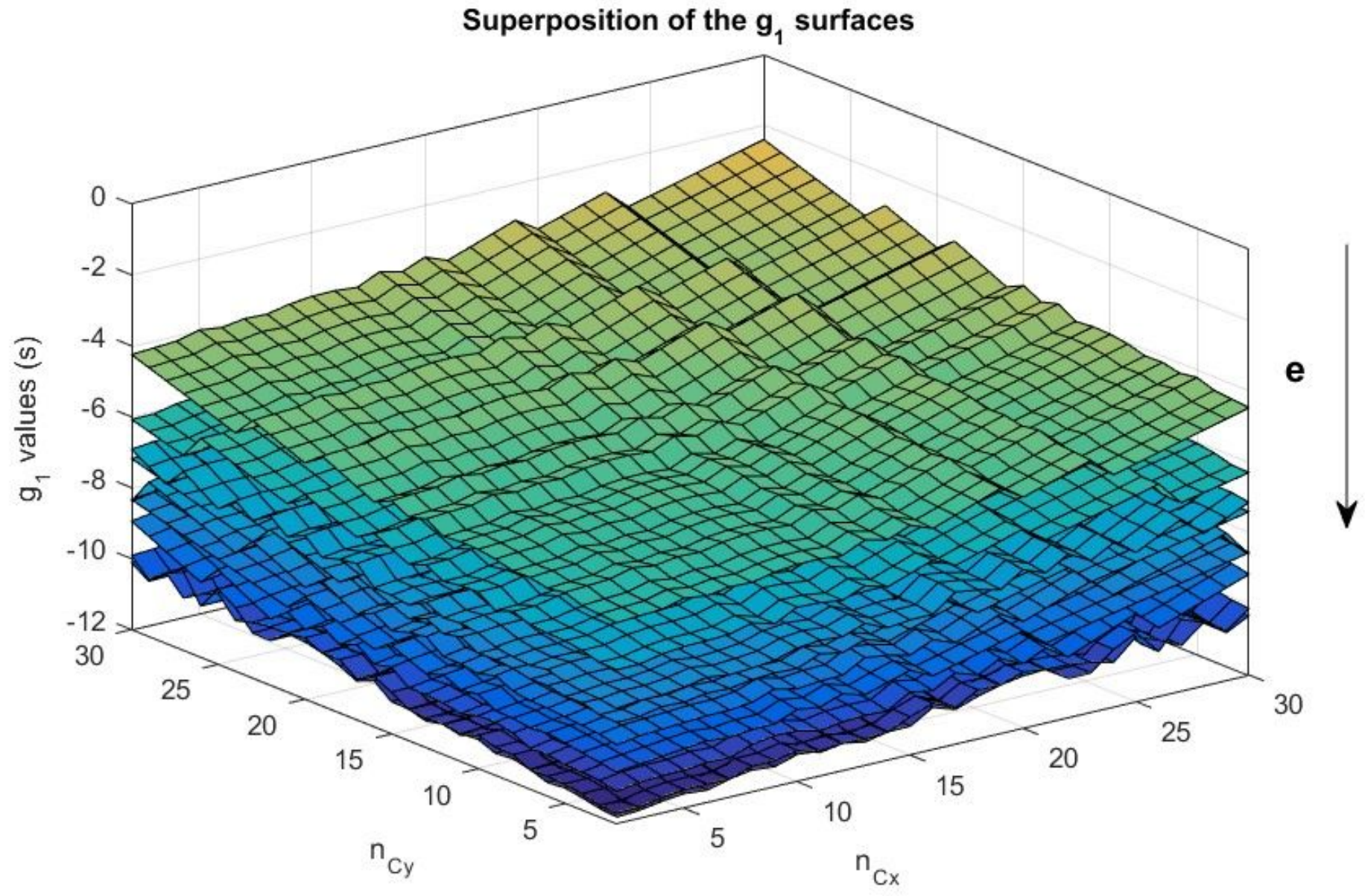

Figure 14

Superposition of the g_1 surfaces according to (n_Cx, n_Cy, e) 


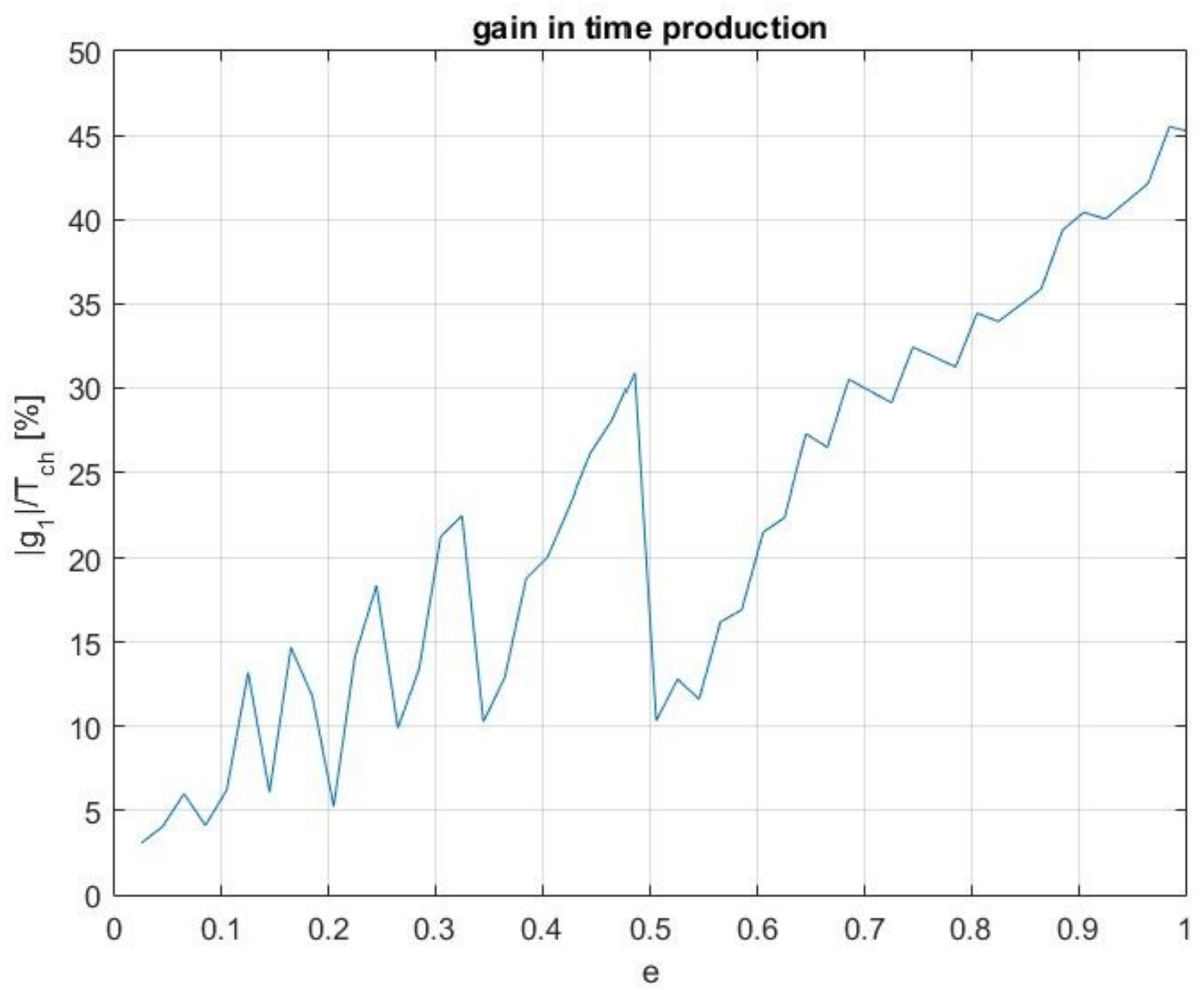

Figure 15

Gain in term of time of production 

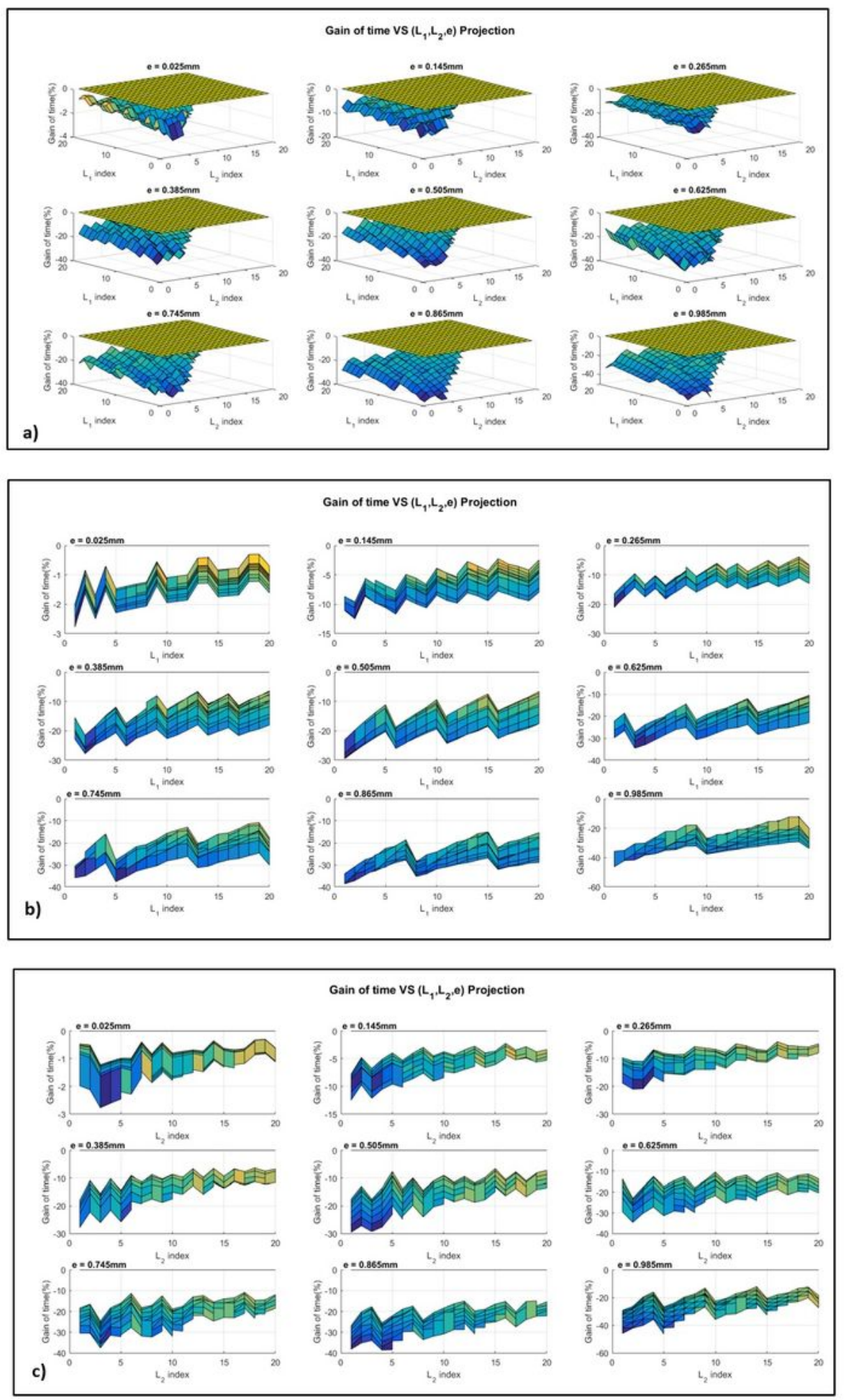

\section{Figure 16}

Gain of time of production according to $\left(L_{-} 1, L_{-} 2, e\right)$ parameters a) 3D view b) projection parallel to $\left.L_{2} c\right)$ projection parallel to L1 


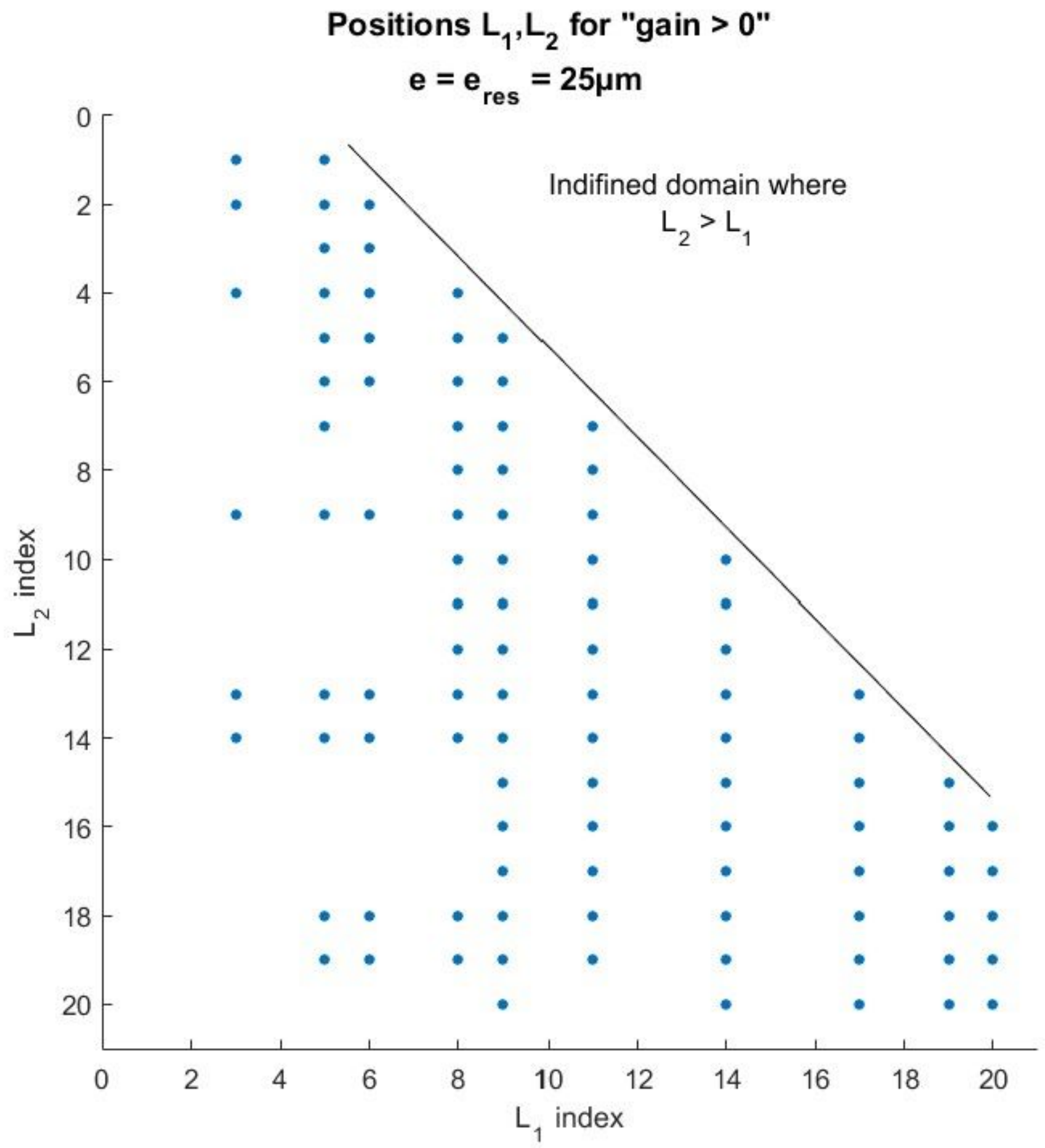

Figure 17

Positions of positive gain in the case of $e=e_{-}$res $=25 \mu \mathrm{m}$ 

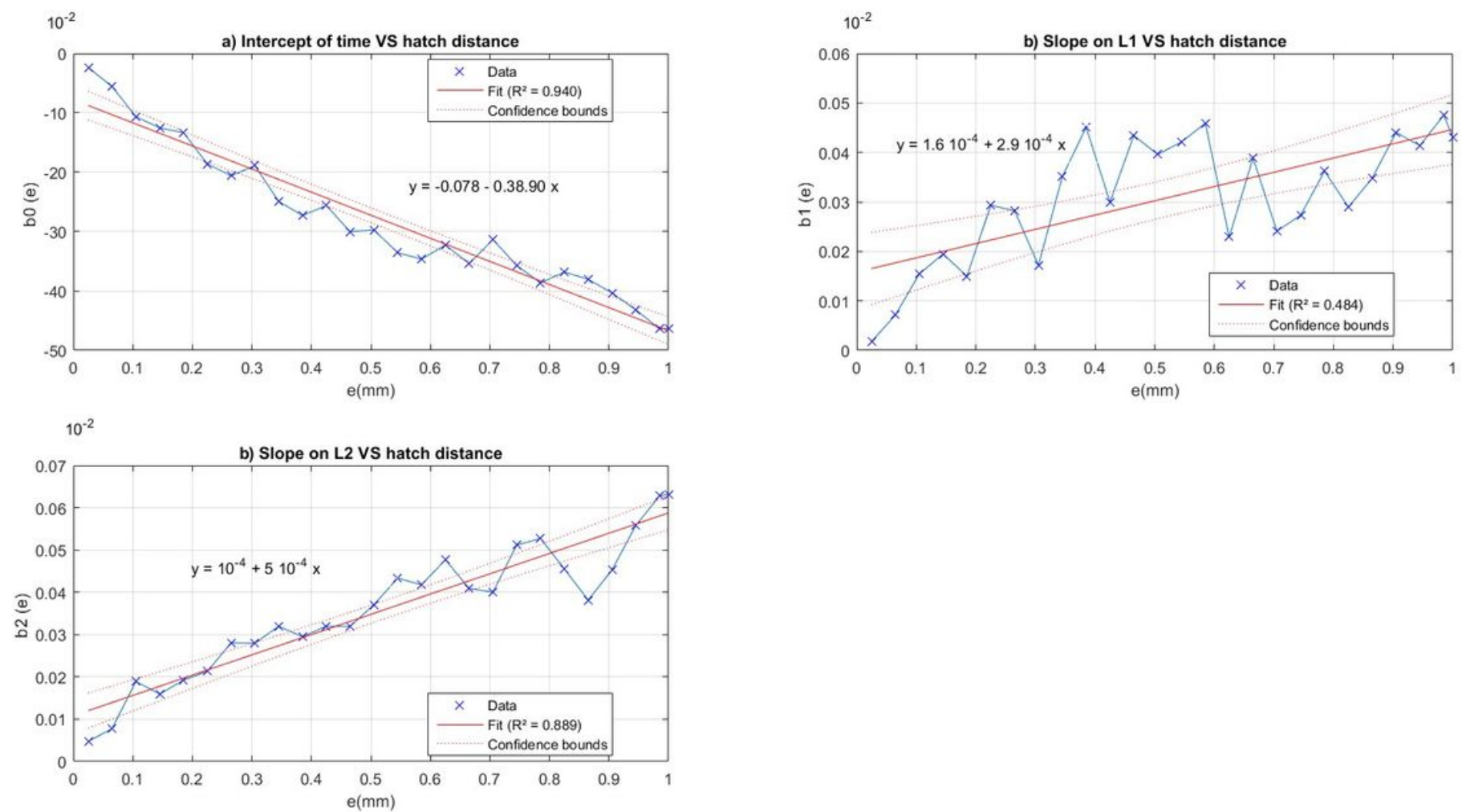

Figure 18

Tendency parameters of gain of time according to the hatch distance $e$ 


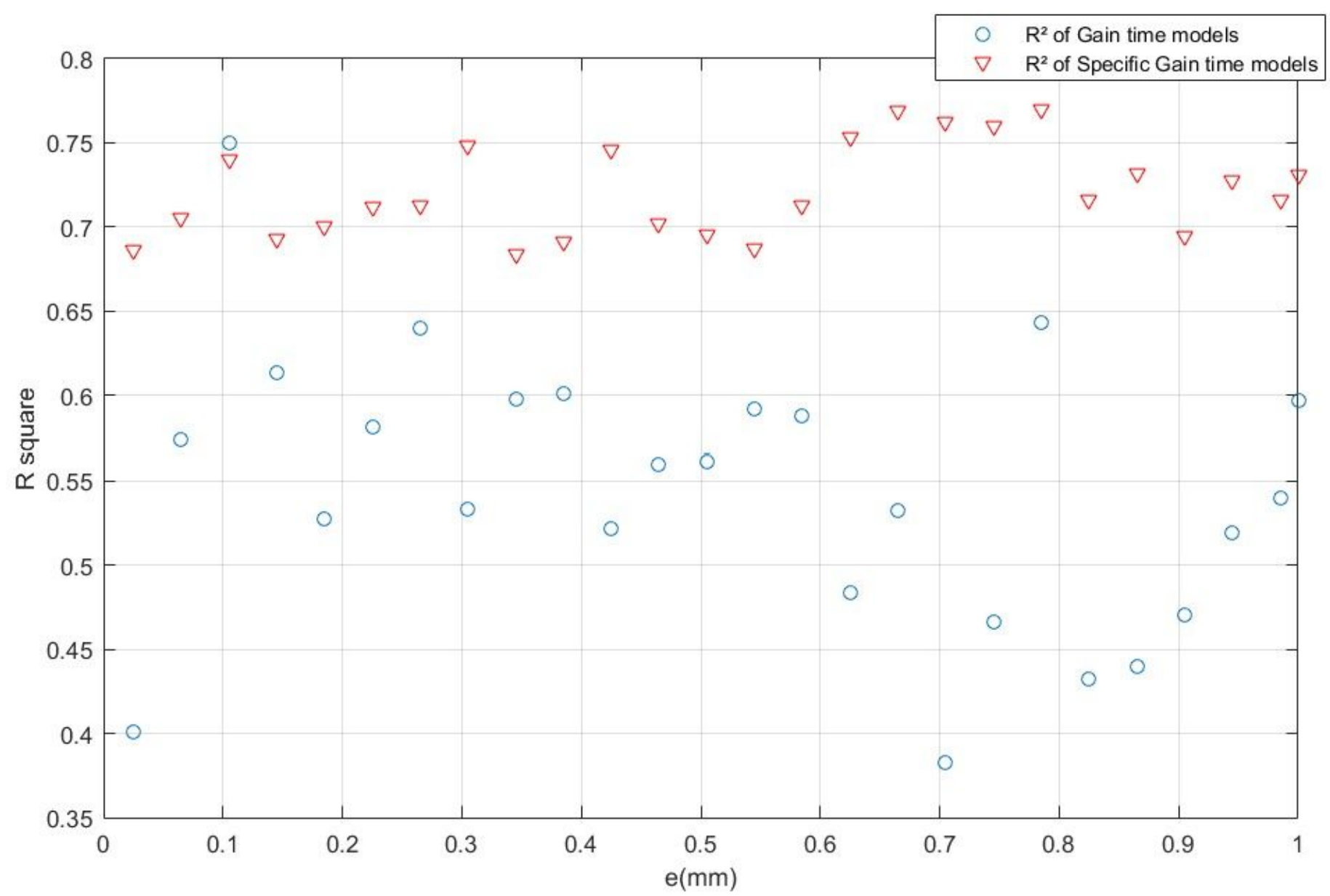

Figure 19

$\mathrm{R}^{2}$ of Gain of Time and SG* surfaces models 
GS : Computed and model
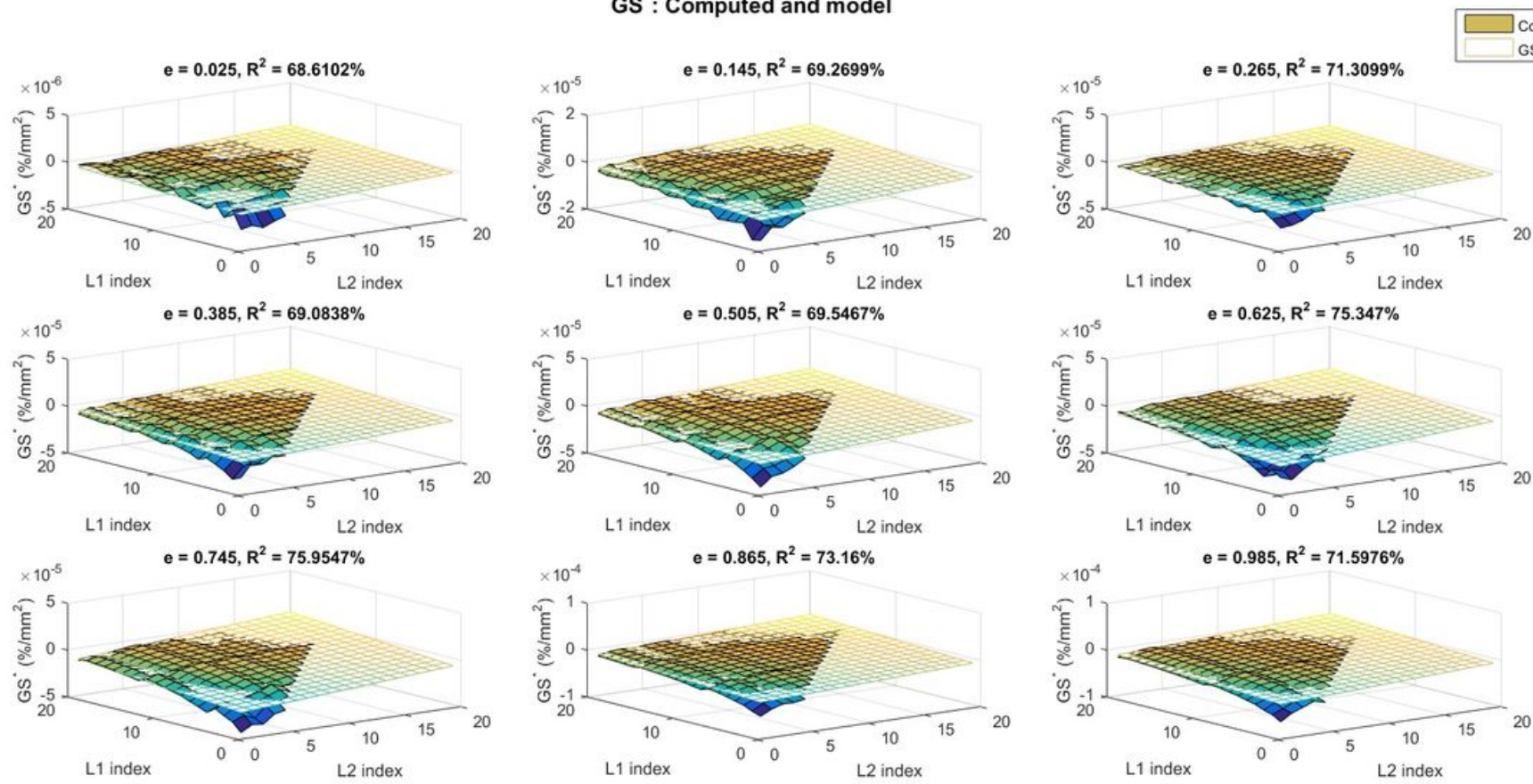

Figure 20

SG* surfaces: Computed and linear models

Tendency parameters of SG ${ }^{*}$
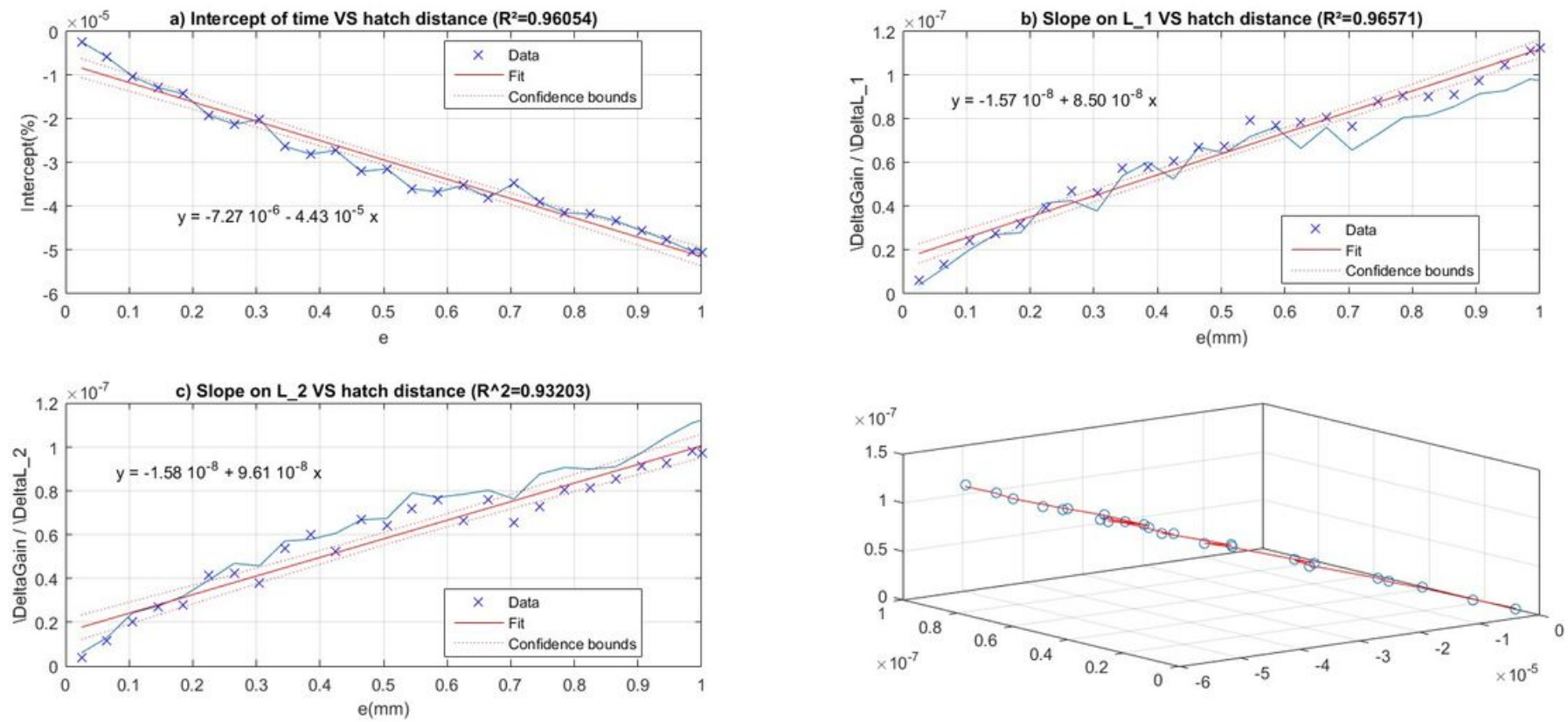

Figure 21

Plot of the linear models of $S G^{\wedge}$ functions a) plot of (e,b_0 (e)) b) plot of (e,b_1 (e)) c) plot of (e,b_2 (e)) 


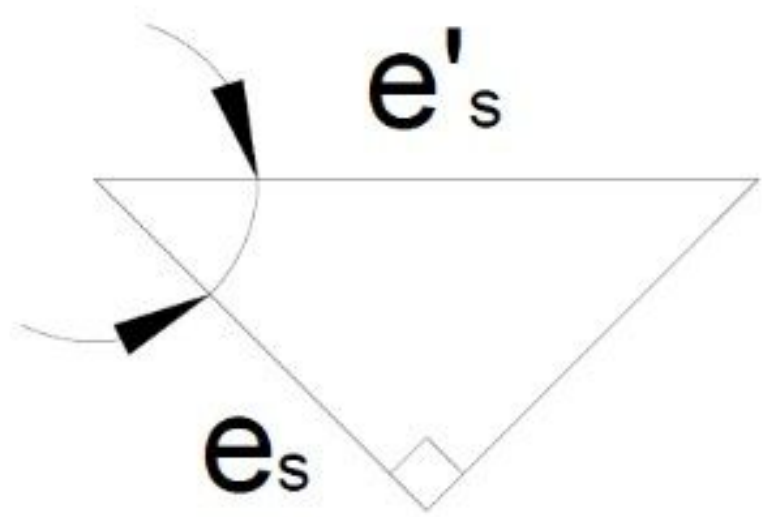

Figure 22

Relation between es and es 